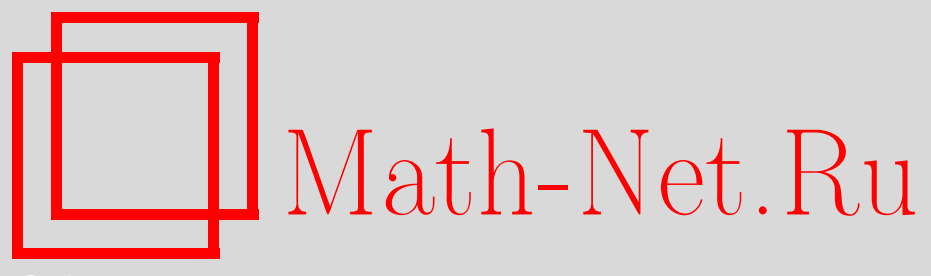

Д. И. Долгопят, Н. И. Чернов, Аномальный ток в периодических газах Лоренца с бесконечным горизонтом, УМН, 2009, том 64, выпуск 4, 73-124

DOI: https://doi.org/10.4213/rm9305

Использование Общероссийского математического портала Math-Net.Ru подразумевает, что вы прочитали и согласны с пользовательским соглашением http://www . mathnet.ru/rus/agreement

Параметры загрузки:

IP : 54.174 .149 .18

26 апреля 2023 г., 18:02:04

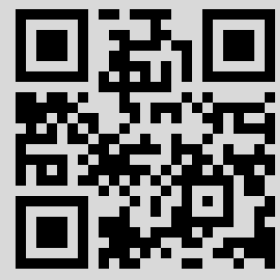




\section{Аномальный ток в периодических газах Лоренца с бесконечным горизонтом}

\section{Д. И. Долгопят, Н. И. Чернов}

Мы изучаем электрический ток в двумерном периодическом газе Лоренца в присутствии слабого однородного электрического поля. Когда горизонт конечен, т. е. длина свободного пробега между столкновениями ограничена, возникающий в результате ток $\mathbf{J}$ пропорционален разнице потенциалов $\mathbf{E}$, т. е. $\mathbf{J}=\frac{1}{2} \mathbf{D}^{*} \mathbf{E}+o(\|\mathbf{E}\|)$, где $\mathbf{D}^{*}-$ матрица диффузии частицы Лоренца, свободно движущейся в отсутствие электрического поля (см. математическое доказательство в [1]). Эта формула согласуется с классическим законом Ома и соотношением Эйнштейна. В настоящей работе исследуется более сложная модель с бесконечным горизонтом. Установлено, что бесконечные коридоры между рассеивателями позволяют частицам (электронам) двигаться быстрее, что приводит к возникновению аномального тока (вызывая "сверхпроводимость"). Точнее, теперь ток задается формулой $\mathbf{J}=\frac{1}{2} \mathbf{D E}|\log \|\mathbf{E}\||+\mathscr{O}(\|\mathbf{E}\|)$, где $\mathbf{D}$ - матрица “супердиффузии" частицы Лоренца, свободно движущейся в отсутствие электрического поля. Это означает, что в этом режиме закон Ома нарушается, но соотношение Эйнштейна (понимаемое должным образом) все же выполняется. Также получены новые результаты для газа Лоренца с бесконечным горизонтом и без внешних полей, дополняющие недавние исследования Д. Саса и Т. Варью [2].

Библиография: 31 название.

Ключевые слова: газ Лоренца, биллиарды, диффузия, электрический ток, закон Ома.

СоДЕРЖАНИЕ

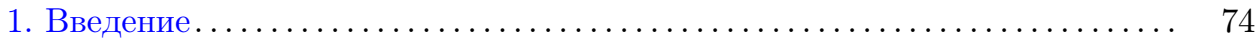

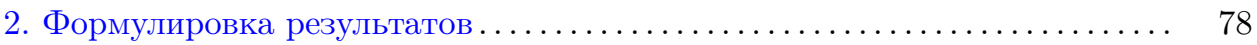

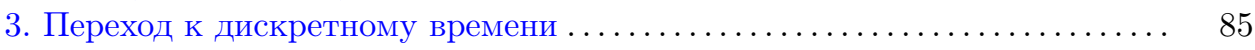

4. План доказательства теоремы $9 \ldots \ldots \ldots \ldots \ldots \ldots \ldots \ldots \ldots \ldots \ldots \ldots \ldots . \ldots \ldots$

Авторы благодарны за гостеприимство Институту Эрвина Шрёдингера в Вене, где была начата эта работа, а Н. И. Чернов благодарен за гостеприимство Университету Мэриленда, где эта работа была завершена. Мы благодарим П. Балинта за чтение рукописи и ценные предложения и Т. Джильберта за полезные обсуждения. Н. И. Чернов был частично поддержан грантом NSF DMS-0652896. Д. И. Долгопят был частично поддержан грантом NSF DMS-0555743.

(С) Д. И. Долгопят, Н. И. Чернов, 2009 


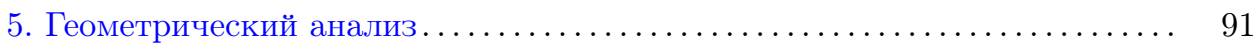

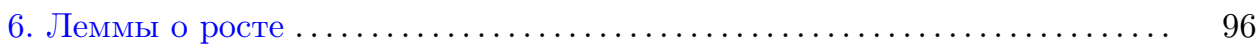

7. Эргодические и статистические свойства .................... 100

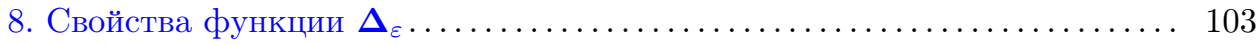

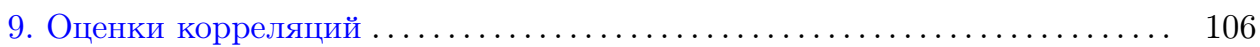

10. Окончание доказательства теоремы $9 \ldots \ldots \ldots \ldots \ldots \ldots \ldots \ldots \ldots \ldots \ldots \ldots$

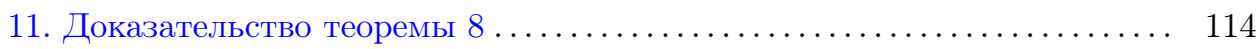

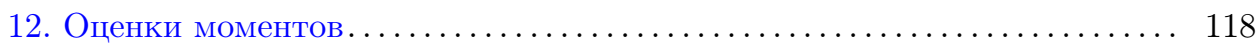

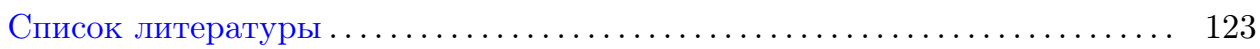

\section{1. Введение}

Газ Лоренца является популярной моделью в математической физике, введенной в 1905 г. (ср. [3]) с целью описания движения электронов в металлах. Исходная модель состояла из малых (точечных) невзаимодействующих частиц, движущихся в пространстве и отскакивающих от неподвижных твердых тел (рассеивателей). В математических исследованиях рассеиватели обычно расположены в узлах периодической решетки, а частицы зеркально отражаются от их поверхностей.

Мы изучаем двумерный периодический газ Лоренца, который сводится к динамической системе, порожденной точечной частицей, движущейся на плоскости $\mathbb{R}^{2}$ и отскакивающей от периодического массива неподвижных выпуклых фигур (играющих роль рассеивателей). На протяжении большей части этой работы мы предполагаем, что “горизонт" бесконечен, т. е. имеются бесконечно длинные коридоры, простирающиеся между рассеивателями; см. рис. 1 в разделе 2.

Математическое изучение двумерных газов Лоренца без внешних полей, т. е. когда частица движется свободно между столкновениями, было начато в 1970 г. Я. Г. Синаем [4]. Благодаря отсутствию внешних сил эта модель является гамильтоновой системой, сохраняющей равновесную меру (Лиувилля). Скорость частицы остается постоянной и традиционно полагается равной единице. В силу периодичности массива рассеивателей динамика сводится к биллиарду на торе с конечным множеством неподвижных рассеивателей.

В [4] доказано, что если рассеиватели являются выпуклыми областями с $C^{3}$-гладкими границами ненулевой кривизны, то получающийся биллиардный поток и отображение столкновений (называемое также производным отображением) гиперболичны (т. е. имеют ненулевые показатели Ляпунова), эргодичны и K-перемешивающие. Позднее было показано, что они обладают свойством Бернулли [5]. Таким образом, эта модель высоко хаотична во всех математических смыслах.

Однако глобальные статистические свойства периодического газа Лоренца, оказывается, зависят от того, конечен или бесконечен горизонт, т. е. ограничен или нет свободный пробег между столкновениями с рассеивателями. Случай конечного горизонта изучен гораздо лучше; ниже мы обсудим его основные свойства. 
Пусть $\widetilde{\mathscr{D}}$ обозначает область, доступную движущейся частице (плоскость $\mathbb{R}^{2}$ минус объединение рассеивателей). Поскольку рассеиватели расположены периодически, всю плоскость можно покрыть копиями некоторого участка (фундаментальной области) $\mathscr{K} \subset \mathbb{R}^{2}$, причем $\widetilde{\mathscr{D}}$ в свою очередь станет объединением копий ограниченной области $\mathscr{D}=\widetilde{\mathscr{D}} \cap \mathscr{K}$. Теперь бесполевой газ Лоренца сводится к плоскому биллиарду на конечном столе $\mathscr{D}$ с периодическими граничными условиями.

Для биллиарда в $\mathscr{D}$ обозначим через $\mathbf{q}(t) \in \mathscr{D}$ и $\mathbf{v}(t) \in \mathbb{S}^{1}$ положение и вектор скорости движущейся частицы в момент времени $t$. Биллиард порождает поток $\Phi^{t}$ на компактном фазовом пространстве $\Omega=\mathscr{D} \times \mathbb{S}^{1}$, который сохраняет меру Лиувилля $\mu_{0}$ (последняя - это просто равномерная мера на $\Omega$ ). Пространство столкновений (называемое также краем или граничным фазовым пространством) определяется как

$$
\mathscr{M}=\{(\mathbf{q}, \mathbf{v}): \mathbf{q} \in \partial \mathscr{D},\|\mathbf{v}\|=1, \mathbf{v} \text { направлен внутрь } \mathscr{D}\},
$$

а отображение столкновений (называемое также "производным отображением") $\mathscr{F}: \mathscr{M} \rightarrow \mathscr{M}$ переводит точку столкновения в следующую точку столкновения.

Обозначим через $(r, \varphi)$ стандартные координаты в $\mathscr{M}$, где $r$ - параметр длины дуги на $\partial \mathscr{D}$, а $\varphi \in[-\pi / 2, \pi / 2]$ - угол между выходящим вектором скорости v и вектором внешней нормали к $\partial \mathscr{D}$ в точке столкновения q (cp. [6], [7]). Отображение $\mathscr{F}$ сохраняет гладкую вероятностную меру $\nu_{0}$ на $\mathscr{M}$, заданную равенствами

$$
d \nu_{0}=c_{\nu} \cos \varphi d r d \varphi, \quad c_{\nu}=[2 \cdot \text { длина }(\partial \mathscr{D})]^{-1} ;
$$

здесь $c_{\nu}$ - нормирующий множитель. Для каждого $X \in \mathscr{M}$ обозначим через

$$
\tau(X)=\min \left\{t>0: \Phi^{t}(X) \in \mathscr{M}\right\}
$$

время первого столкновения траектории, начинающейся в $X$.

Для частицы, движущейся в бесконечной области $\widetilde{\mathscr{D}}$, обозначим через $\widetilde{\mathbf{q}}(t)$ ее положение в момент времени $t$, а через $\widetilde{\mathbf{q}}_{n}-$ ее положение в момент $n$-го столкновения с $\partial \widetilde{\mathscr{D}}$. Обозначим через $\boldsymbol{\Delta}_{n}=\widetilde{\mathbf{q}}_{n+1}-\widetilde{\mathbf{q}}_{n}$ вектор смещения между столкновениями. (Заметим, что мы используем волну для обозначений, относящихся к динамике в неограниченной области $\widetilde{\mathscr{D}}$.)

Во всех теоремах, кроме теоремы 3 , предполагается, что начальное положение $\widetilde{\mathbf{q}}(0)$ и начальный вектор скорости $\mathbf{v}(0)$ выбраны в соответствии с мерой Лиувилля $\mu_{0}$ в $\mathscr{D} \times \mathbb{S}^{1}$. Все результаты остаются справедливыми, если начальное распределение гладкое и имеет компактный носитель.

ТЕОРема 1 [8], [6]. Предположим, что частица движется свободно в отсутствие внешних полей.

(а) Величина $\widetilde{\mathbf{q}}(t) / \sqrt{t}$ при $t \rightarrow \infty$ сходится по распределению к нормальному распределению,

$$
\frac{\widetilde{\mathbf{q}}(t)}{\sqrt{t}} \Rightarrow \mathscr{N}\left(\mathbf{0}, \mathbf{D}^{*}\right),
$$

с невырожденной ковариащионной матрищей $\mathbf{D}^{*}$, называемой матрицей диффузии. 
(б) Матрица диффузии $\mathbf{D}^{*}$ задается формулой Грина-Кубо:

$$
\mathbf{D}^{*}=\frac{1}{\bar{\tau}_{0}} \sum_{n=-\infty}^{\infty} \nu_{0}\left(\boldsymbol{\Delta}_{0} \otimes \boldsymbol{\Delta}_{n}\right),
$$

где $\bar{\tau}_{0}=\nu_{0}(\tau)$ - средняя длина свободного пробега, задаваемая равенством

$$
\bar{\tau}_{0}=\nu_{0}(\tau)=\frac{\pi \cdot \text { площадь }(\mathscr{D})}{\text { длина }(\partial \mathscr{D})},
$$

a $\mathbf{u} \otimes \mathbf{v}$ обозначает “тензорное произведение” двух векторов, т.е. произведение вектор-столбца $\mathbf{u}$ и вектор-строки $\mathbf{v}$.

Эта теорема была выведена в [8], [6]. Л.-С. Янг [9] показала, что ряд (1.3) сходится экспоненциально быстро; см. недавнее изложение в [7; гл. 7]; доказательство формулы (1.4) см. в [7; 2.13$]$.

Далее рассмотрим двумерные периодические газы Лоренца с конечным горизонтом, в которых на частицу действует малое однородное (т. е. постоянное во времени и пространстве) внешнее поле Е. Чтобы энергия частицы сохранялась, поле Е спаривают с гауссовым термостатом [1], [10], [11], так что движение между столкновениями подчиняется уравнениям

$$
\frac{d \widetilde{\mathbf{q}}}{d t}=\mathbf{v}, \quad \frac{d \mathbf{v}}{d t}=\mathbf{E}-\zeta \mathbf{v},
$$

где $\zeta=\langle\mathbf{E}, \mathbf{v}\rangle /\|\mathbf{v}\|^{2}$. Член трения $\zeta \mathbf{v}$ называется гауссовым термостатом; он обеспечивает выполнение равенства $\|\mathbf{v}\|=$ const во все моменты времени; мы будем опять предполагать, что $\|\mathbf{v}\|=1$.

Зафиксируем направление поля $\mathbf{E}$ и будем считать $\varepsilon=\|\mathbf{E}\|$ малым параметром модели. Снова в силу периодичности рассеивателей получаем новый поток, обозначаемый $\Phi_{\varepsilon}^{t}$, на том же фазовом пространстве $\Omega=\mathscr{D} \times \mathbb{S}^{1}$ и новое отображение столкновений $\mathscr{F}: \mathscr{M} \rightarrow \mathscr{M}$; пространства $\Omega$ и $\mathscr{M}$ остаются независимыми от Е. Функция момента столкновения (1.2) теперь зависит от $\varepsilon$, т. е. $\tau=\tau_{\varepsilon}$. Также для любого $X \in \mathscr{M}$ обозначим через

$$
\boldsymbol{\Delta}_{\varepsilon}(X)=\widetilde{\mathbf{q}}_{1}-\widetilde{\mathbf{q}}_{0}
$$

смещение частицы, движущейся в бесконечной области $\widetilde{\mathscr{D}}$, до ее следующего столкновения с $\partial \widetilde{\mathscr{D}}$.

ТЕОРема 2 [1], [10]. Пусть $\widetilde{\mathbf{q}}(t)$ - положение частицы газа Лоренца с конечным горизонтом и с малым внешним полем $\mathbf{E}$, спаренным с гауссовым термостатом (1.5). Тогда справедливы следующие утверждения.

(а) Для достаточно малого $\varepsilon=\|\mathbf{E}\|$ отображение $\mathscr{F} \varepsilon$ гиперболично; оно сохраняет (SRB-) меру Синая-Рюэля-Боуэна (установившееся состояние) $\nu_{\varepsilon}$, которая эргодична, перемешивающая, сингулярна относительно меры Лебега, но положительна на открытых множествах. Поток $\Phi_{\varepsilon}^{t}$ также сохраняет $S R B$-меру $\mu_{\varepsilon}$ на $\Omega$, которая эргодична, перемешивающая и положительна на открытых множествах. Электрический ток

$$
\mathbf{J}=\lim _{t \rightarrow \infty} \frac{\widetilde{\mathbf{q}}(t)}{t}=\mu_{\varepsilon}(\mathbf{v})=\frac{\nu_{\varepsilon}\left(\boldsymbol{\Delta}_{\varepsilon}\right)}{\bar{\tau}_{\varepsilon}}
$$


корректно определен; здесъ $\bar{\tau}_{\varepsilon}=\nu_{\varepsilon}\left(\tau_{\varepsilon}\right)$ - средняя длина свободного пробега (или среднее время между столкновениями), для которой $\bar{\tau}_{\varepsilon}=\bar{\tau}_{0}+\mathscr{O}(\varepsilon)$ при $\varepsilon \rightarrow 0$.

(б) Имеет место равенство

$$
\mathbf{J}=\frac{1}{2} \mathbf{D}^{*} \mathbf{E}+o(\varepsilon)
$$

где $\mathbf{D}^{*}$ - матрица диффузии из теоремы 1.

(в) Имеет место следующая слабая сходимость при $t \rightarrow \infty$ :

$$
\frac{\widetilde{\mathbf{q}}(t)-\mathbf{J} t}{\sqrt{t}} \Rightarrow \mathscr{N}\left(0, \mathbf{D}_{\varepsilon}^{*}\right),
$$

где $\mathbf{D}_{\varepsilon}^{*}-$ coответствующая матрица диффузии.

(г) Матрица диффузии непрерывна по $\varepsilon$ в точке $\varepsilon=0$ :

$$
\mathbf{D}_{\varepsilon}^{*}=\mathbf{D}^{*}+o(1) .
$$

Эта теорема была доказана в [1], [10] и распространена на более общие внешние силы в [12], [13].

Заметим, что (1.7) можно рассматривать [1], [10] как классический закон Ома: электрический ток $\mathbf{J}$ пропорционален напряжению $\mathbf{E}$ (в старшем порядке). Тот факт, что электрическая проводимость, т. е. $\frac{1}{2} \mathbf{D}^{*}$ в $(1.7)$, пропорциональна матрице диффузии $\mathbf{D}^{*}$, известен в физике как соотношение Эйнштейна [1], [10]. Заметим, что в силу (1.8) соотношение Эйнштейна может быть переформулировано как

$$
\mathbf{J}=\frac{1}{2} \mathbf{D}_{\varepsilon}^{*} \mathbf{E}+\text { члены более высокого порядка. }
$$

Согласно теореме 2, положение частицы можно грубо аппроксимировать следующим образом:

$$
\widetilde{\mathbf{q}}(t) \approx\left[\frac{1}{2} \mathbf{D}^{*}+o(1)\right] \mathbf{E} t+\left[\left(\mathbf{D}^{*}\right)^{1 / 2}+o(1)\right] Z \sqrt{t},
$$

где $Z$ обозначает двумерный стандартный нормальный случайный вектор. Первый член в (1.10) представляет установившийся снос частицы, а второй ее хаотичную диффузию.

Обратимся далее к частице Лоренца, движущейся во внешнем поле без термостата, т. е. уравнения движения теперь очень простые:

$$
\frac{d \widetilde{\mathbf{q}}}{d t}=\mathbf{v}, \quad \frac{d \mathbf{v}}{d t}=\mathbf{E} .
$$

Однако этот случай намного сложнее предыдущего, потому что частице разрешается ускоряться (“нагреваться"), так что система уже не сохраняет конечную меру. Если выбрать систему координат так, чтобы ось $x$ была направлена вдоль поля $\mathbf{E}$, т. е. $\mathbf{E}=(\varepsilon, 0)$, то можно ожидать, что $x(t) \rightarrow \infty$ и $\|\mathbf{v}(t)\| \rightarrow \infty$ при $t \rightarrow \infty$. Точнее, так как полная энергия

$$
H=\frac{1}{2}\|\mathbf{v}(t)\|^{2}-\langle\mathbf{E}, \widetilde{\mathbf{q}}(t)\rangle=\frac{1}{2}\|\mathbf{v}(t)\|^{2}-\varepsilon x(t)
$$


сохраняется, имеем $x(t) \sim\|\mathbf{v}(t)\|^{2}$. В физической литературе давно высказывалась гипотеза, что $x(t) \sim t^{2 / 3}$ и $\|\mathbf{v}(t)\| \sim t^{1 / 3}$ (см. ссылки в [14], [15]), но математически эта модель была исследована только совсем недавно [15].

Предположим, что частица заключена в полуплоскости $\{x \geqslant 0\}$ и испытывает зеркальные отражения от прямой $x=0$. Собственно, эта модель известна в физике как доска Гальтона [16].

ТЕОРема 3 [15]. Предположим, что начальное условие частицы имеет гладкую плотность с компактным носителем на энергетической поверхности $\left\{\mathrm{H}=\mathrm{H}_{0}\right\}$, где $\mathrm{H}_{0}$ достаточно велико.

(a) Найдется такая константа $c_{\diamond}>0$, что $c_{\diamond} t^{-2 / 3} x(t)$ сходится при $t \rightarrow \infty$ $\kappa$ случайной величине с плотностъю

$$
\frac{3}{2 \Gamma(2 / 3)} \exp \left[-z^{3 / 2}\right], \quad z \geqslant 0 .
$$

(б) Константа $c_{\diamond}$ задается равенством

$$
c_{\diamond}=\left(\frac{32\|\mathbf{E}\|^{5}}{81\left\langle\mathbf{D}^{*} \mathbf{E}, \mathbf{E}\right\rangle}\right)^{1 / 3} .
$$

ЗАмечАниЕ. (в) Для $t^{-2 / 3} y(t)$ также существует предельное распределение, но оно задается более сложной формулой [14].

Итак, в зависимости от типа внешних сил частица, движущаяся в газе Лоренца с конечным горизонтом, может проявлять различное качественное поведение. Но во всех случаях коэффициенты переноса удовлетворяют простым соотношениям и выражаются в терминах матрицы диффузии $\mathbf{D}^{*}$. Заметим, что матрица $\mathbf{D}^{*}$, заданная в (1.3), является весьма неявной функцией геометрии рассеивателей. Действительно, есть указания [17] на то, что $\mathbf{D}^{*}$ может не быть дифференцируемой функцией относительно геометрических характеристик рассеивателей (например, их центров или диаметров). Но для данного $\mathbf{D}^{*}$ совсем нетрудно вычислить другие коэффициенты переноса.

Этим мы завершаем вводный обзор газов Лоренца с конечным горизонтом. В следующем разделе мы обратимся к более сложному случаю бесконечного горизонта.

\section{2. Формулировка результатов}

Эта работа является частью большого проекта по распространению всех вышеупомянутых результатов на периодические газы Лоренца с бесконечным горизонтом. В этой работе мы приводим аналоги теорем 1 и 2, оставляя распространение теоремы 3 для последующей публикации.

Свободный газ Лоренца. В случае бесконечного горизонта частица может свободно двигаться без столкновений вдоль бесконечных коридоров, простирающихся между рассеивателями, см. рис. 1. Теперь ее долговременное поведение совершенно отлично от описанного в теореме 1. Первое проявление этой разницы состоит в том, что ряд Грина-Кубо (1.3), определяющий матрицу диффузии в случае конечного горизонта, теперь расходится, так как его 


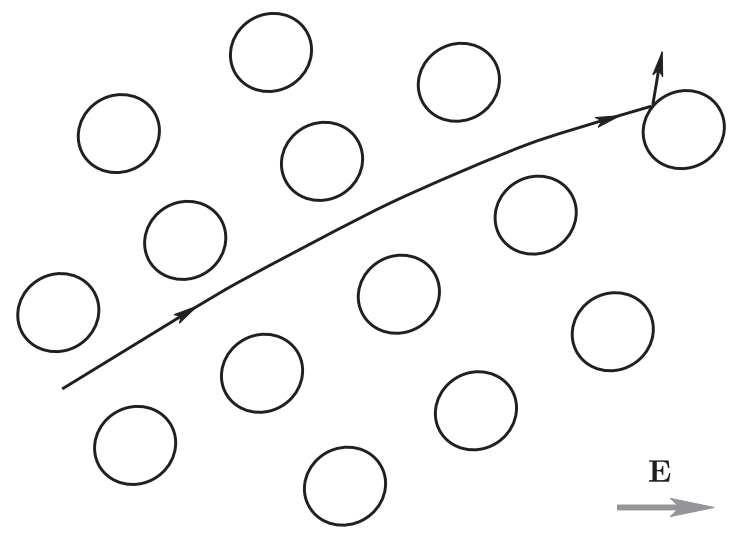

Рис. 1. Частица, движущаяся вдоль бесконечного коридора между рассеивателями: благодаря внешнему полю $\mathbf{E}$ ее траектория изгибается и попадает на рассеиватель

центральный член бесконечен:

$$
\nu_{0}\left(\boldsymbol{\Delta}_{0} \otimes \boldsymbol{\Delta}_{0}\right)=\infty
$$

(Основной технический результат нашей работы, однако, утверждает, что все остальные члены, $\nu_{0}\left(\boldsymbol{\Delta}_{0} \otimes \boldsymbol{\Delta}_{n}\right)$ при $n \neq 0$, конечны и убывают экспоненциально по $n$; см. предложение 9.1.)

Поскольку матрица диффузии, определяемая рядом (1.3), становится бесконечной, у частицы появляется аномальная диффузия (часто называемая супердиффузией). А именно, правильный нормирующий множитель в центральной предельной теореме теперь равен $\sqrt{t \log t}$, а не $\sqrt{t}$. Этот факт давно высказывался в виде гипотезы, а частичное его доказательство было опубликовано П. Блехером [18] в 1992 г. Полное математическое доказательство - для дискретного времени - появилось в 2007 г. в работе Д. Саса и Т. Варью [2]. Их главный результат - следующая теорема.

ТеОрема 4 [2]. Пусть $\widetilde{\mathbf{q}}_{n}$ - положение частицы в момент $n$-го столкновения в газе Лоренца с бесконечным горизонтом и без внешних полей.

(а) Имеет место следующая слабая сходимость при $n \rightarrow \infty$ :

$$
\frac{\widetilde{\mathbf{q}}_{n}}{\sqrt{n \log n}} \Rightarrow \mathscr{N}(0, \widehat{\mathbf{D}}),
$$

где $\widehat{\mathbf{D}}$ называется матрицей супердиффузии для дискретного времени.

(б) Матрица $\widehat{\mathbf{D}}$ невырождена, если есть два непараллелъных коридора.

Матрица $\widehat{\mathbf{D}}$ задается простой явной формулой в терминах геометрических характеристик бесконечных коридоров. А именно, пусть каждый бесконечный коридор в $\widetilde{D}$ ограничен двумя прямыми, каждая из которых касается бесконечного ряда рассеивателей, являющихся копиями рассеивателя в $\mathscr{D}$; 
тогда такие прямые являются траекториями некоторых неподвижных точек $X \in \mathscr{M}: \mathscr{F}(X)=X$. Теперь имеем

$$
\widehat{\mathbf{D}}=\sum \frac{c_{\nu} w_{X}^{2}}{2\|\boldsymbol{\Delta}(X)\|} \boldsymbol{\Delta}(X) \otimes \boldsymbol{\Delta}(X),
$$

где сумма берется по всем коридорам, $\boldsymbol{\Delta}(X)=\boldsymbol{\Delta}_{0}(X)$ - вектор смещения точки $X$, введенный ранее, а $w_{X}$ - ширина коридора, ограниченного траекторией точки $X$.

Наш первый результат - вариант этой теоремы для непрерывного времени, который также включает в себя слабый принцип инвариантности. Напомним, что начальное положение $\widetilde{\mathbf{q}}(0)$ и начальный вектор скорости $\mathbf{v}(0)$ всегда выбираются в соответствии с гладким распределением с компактным носителем.

Теорема 5. Пусть $\widetilde{\mathbf{q}}(t)$ - положение частицы в газе Лорениа с бесконечным горизонтом и без внешних полей.

(а) Имеет место следующая слабая сходимость при $t \rightarrow \infty$ :

$$
\frac{\widetilde{\mathbf{q}}(t)}{\sqrt{t \log t}} \Rightarrow \mathscr{N}(0, \mathbf{D}),
$$

где $\mathbf{D}$ называется матрицей супердиффузии.

(б) Для последней матрицы справедливо равенство $\mathbf{D}=\bar{\tau}_{0}^{-1} \widehat{\mathbf{D}}$, где $\bar{\tau}_{0}-$ средняя длина свободного пробега, заданная в (1.4). В частности, D невырождена тогда и только тогда, когда имеется два непараллельных коридора.

(в) При этом в последнем случае (когда $\mathbf{D}$ невырождена) имеет место слабый принцип инвариантности, т.е. прочесс

$$
\frac{\widetilde{\mathbf{q}}(s T)}{\sqrt{T \log T}}, \quad 0<s<1,
$$

сходится при $T \rightarrow \infty \kappa$ броуновскому движению с нулевым средним и ковариационной матрищей $\mathbf{D}$.

Случай параллельных коридоров рассматривается отдельно, см. ниже. По ходу доказательства теоремы 5 (см. разделы 11,12 ) будет также дано другое доказательство теоремы 4, отличное от опубликованного в [2].

Газ Лоренца под действием внешнего поля. Наша главная цель - показать, что в двумерном периодическом газе Лоренца с бесконечным горизонтом и малым постоянным внешним полем $\mathbf{E}$, спаренным с гауссовым термостатом, электрический ток также анормален. А именно, если снова обозначить $\varepsilon=\|\mathbf{E}\|$, то поток $\mathbf{J}$ будет пропорционален $\varepsilon|\log \varepsilon|$, а не $\varepsilon$.

Точнее, теперь есть два различных случая. Во-первых, если поле $\mathbf{E}$ параллельно одному из бесконечных коридоров, то с положительной вероятностью частица попадет в этот коридор и затем продолжит движение "по баллистической траектории", без столкновений. В этом случае $\widetilde{\mathbf{q}}(t) / t$ сходится к 1 при $t \rightarrow \infty$ (независимо от $\varepsilon$ ), и это тривиальный случай.

Более интересна ситуация общего положения, в которой поле $\mathbf{E}$ не параллельно ни одному из коридоров. Тогда длина свободного пробега оказывается ограниченной, так как траектория частицы изгибается под действием поля и обязательно выйдет из любого коридора (см. рис. 1). На самом деле мы покажем, что максимальная длина свободного пробега между столкновениями является $\mathscr{O}\left(\varepsilon^{-1 / 2}\right)$. 
Как и ранее, мы фиксируем направление поля $\mathbf{E}$ и считаем $\varepsilon=\|\mathbf{E}\|$ малым параметром модели. В силу периодичности получаем поток $\Phi_{\varepsilon}^{t}$ на $\Omega=\mathscr{D} \times \mathbb{S}^{1}$ и отображение столкновений $\mathscr{F}_{\varepsilon}: \mathscr{M} \rightarrow \mathscr{M}$. Напомним, что $\tau=\tau_{\varepsilon}$ обозначает время первого столкновения (1.2), а $\boldsymbol{\Delta}_{\varepsilon}$ обозначает смещение перед первым столкновением (1.6). Наш основной результат - следующая теорема.

Теорема 6. Пусть $\widetilde{\mathbf{q}}(t)$ - положение частицы в газе Лоренца с бесконечнъм горизонтом и с малым внешним полем $\mathbf{E}$, спаренным с гауссовым термостатом (1.5). Предположим, что $\mathbf{E}$ не параллельно ни одному из бесконечных коридоров.

(а) Для достаточно малого є отображение столкновений $\mathscr{F}$ гиперболично; оно сохраняет SRB-меру (установившееся состояние) $\nu_{\varepsilon}$, которая эргодична, перемешивающая и положительна на открытых множествах. Поток $\Phi_{\varepsilon}^{t}$ также сохраняет SRB-меру $\mu_{\varepsilon}$ на $\Omega$, которая эргодична, перемешивающая и положительна на открытых множествах. Электрический ток

$$
\mathbf{J}=\lim _{t \rightarrow \infty} \frac{\widetilde{\mathbf{q}}(t)}{t}=\mu_{\varepsilon}(\mathbf{v})=\frac{\nu_{\varepsilon}\left(\boldsymbol{\Delta}_{\varepsilon}\right)}{\bar{\tau}_{\varepsilon}}
$$

корректно определен; здесь $\bar{\tau}_{\varepsilon}=\nu_{\varepsilon}\left(\tau_{\varepsilon}\right)$ - средний свободный пробег (среднее время между столкновениями).

(б) Для тока $\mathbf{J}$ выполнено равенство

$$
\mathbf{J}=\frac{1}{2}|\log \varepsilon| \mathbf{D E}+\delta \mathbf{J},
$$

где $\mathbf{D}$ - матрица супердиффузии из теоремы 5. Остаточный член $\delta \mathbf{J}$ в (2.3) ограничен следующим образом:

$$
\|\delta \mathbf{J}\| \leqslant A_{\mathbf{u}} \varepsilon
$$

где $A_{\mathbf{u}}>0$ зависит только от направления $\mathbf{u}=\mathbf{E} /\|\mathbf{E}\|$ поля.

(в) Имеет место следующая слабая сходимость при $t \rightarrow \infty$ :

$$
\frac{\widetilde{\mathbf{q}}(t)-\mathbf{J} t}{\sqrt{t}} \Rightarrow \mathscr{N}\left(0, \mathbf{D}_{\varepsilon}\right),
$$

где $\mathbf{D}_{\varepsilon}-$ coответствуюшая матрица диффузии.

(г) Для матричы $\mathbf{D}_{\varepsilon}$ выполнено равенство

$$
\mathbf{D}_{\varepsilon}=|\log \varepsilon| \mathbf{D}+\delta \mathbf{D}
$$

где $\|\delta \mathbf{D}\| \leqslant B_{\mathbf{u}}$ и $B_{\mathbf{u}}-$ константа, зависящая только от $\mathbf{u}$.

При этом случайный процесс положения точки $\{\widetilde{\mathbf{q}}(t)-\mathbf{J} t\}$ удовлетворяет стандартному слабому принципу инвариантности, принципу инвариантности почти наверное и закону повторного логарифма; см., например, [7; § 7.9].

Заметим, что скаляры $A_{\mathbf{u}}$ и $B_{\mathbf{u}}$, как функции от $\mathbf{u}$, не являются равномерно ограниченными. На самом деле мы ожидаем, что они растут до бесконечности, когда поле становится почти параллельно одному из бесконечных коридоров (это происходит потому, что тогда ток $\mathbf{J}$, по-видимому, имеет порядок единицы, как отмечено выше, но этот случай здесь не исследуется). 
Из теоремы 6 можно заключить, что аналогично (1.10) положение частицы грубо аппроксимируется следующим образом:

$$
\widetilde{\mathbf{q}}(t) \approx\left[\frac{1}{2}|\log \varepsilon| \mathbf{D}+\mathscr{O}(1)\right] \mathbf{E} t+\left[(|\log \varepsilon| \mathbf{D})^{1 / 2}+\mathscr{O}(1)\right] Z \sqrt{t},
$$

где $Z$ обозначает двумерный стандартный нормальный случайный вектор.

Заметим, что так как сходимость в теореме 6 не равномерная, то (2.7) справедливо только для достаточно больших $t$. Точнее, имеет место следующее предложение.

ПреДЛОЖЕНИЕ 2.1. Предположим, что $t \rightarrow \infty u \varepsilon \rightarrow 0$. Тогда

$$
\frac{\widetilde{\mathbf{q}}(t)-\mathbf{J} t}{\sqrt{t \log \min \left\{t, \varepsilon^{-1}\right\}}} \Rightarrow \mathscr{N}(0, \mathbf{D}) \text {. }
$$

Сравнивая случаи конечного и бесконечного горизонта, сделаем следующие наблюдения.

- Классический закон Ома не выполняется в случае бесконечного горизонта, поскольку электрическая проводимость становится бесконечной.

- Соотношения Эйнштейна в виде (1.9) остаются справедливыми.

- Непрерывность матрицы диффузии теряет смысл, так как матрица диффузии (1.3) становится бесконечной в отсутствие полей. Вместо этого имеется матрица супердиффузии $\mathbf{D}$, которая зависит только от геометрии бесконечных коридоров, и наблюдается следующее поведение. Поле изгибает биллиардные траектории и ограничивает сверху самый длинный свободный пробег на уровне $\mathscr{O}\left(\varepsilon^{-1 / 2}\right)$. Такие большие значения свободного пробега достигаются типичными бесполевыми (биллиардными) траекториями за промежутки времени $t \sim \varepsilon^{-1}$. Поэтому при $t \ll \varepsilon^{-1}$ полем можно пренебречь и эффективные матрицы ковариации примерно одинаковы - что с полем, что без поля. Но при больших промежутках времени, $t \gg \varepsilon^{-1}$, наличие поля приводит к гораздо меньшим флуктуациям по сравнению с бесполевым случаем. Это объясняет, почему имеет место диффузия, когда есть поле, и супердиффузия, когда полей нет.

- Матрица супердиффузии $\mathbf{D}$ допускает простое и явное описание в терминах геометрических параметров бесконечных коридоров (см. (2.1)), что составляет яркий контраст с матрицей диффузии $\mathbf{D}^{*}$, заданной бесконечным рядом (1.3). Однако если рассеиватели становятся настолько малыми, что число коридоров становится большим, то вычисление $\mathbf{D}$ по формуле (2.1) тоже может быть очень сложным; см. также [19], [20].

Особый случай параллельных коридоров. Результаты предыдущего раздела справедливы для любого числа и любой конфигурации коридоров, но они дают неполную картину, когда все коридоры параллельны, так как матрица $\mathbf{D}$ тогда будет вырожденной. Например, теорема 5 в этом случае будет утверждать только, что типичные флуктуации частицы в направлении, перпендикулярном коридорам, являются $o(\sqrt{t \log t})$. С другой стороны, из [21] легко следует, что правильный нормирующий множитель в центральной предельной теореме в этом случае равен $\sqrt{t}$. Ниже мы приводим теорему, специально приспособленную для случая параллельных коридоров.

Для удобства в этой теореме и последующих предложениях система координат выбирается так, чтобы все бесконечные коридоры были параллельны 
оси $x$. Заметим: это отличается от нашего предыдущего соглашения, что ось $x$ параллельна полю Е. Этот новый выбор системы координат принят только в пределах текущего пункта; затем мы вернемся к старому выбору, когда направление $x$ параллельно $\mathbf{E}$.

Теорема 7. Пусть $\widetilde{\mathbf{q}}(t)=(x(t), y(t))$ - положение частицы в газе Лоренца с бесконечным горизонтом и без внешних полей, в котором все бесконечные коридоры параллельны оси $x$.

(а) Имеет место слабая сходимость при $t \rightarrow \infty$

$$
\left(\frac{x(t)}{\sqrt{t \log t}}, \frac{y(t)}{\sqrt{t}}\right) \Rightarrow \mathscr{N}\left(0,\left(\begin{array}{cc}
\mathbf{D}_{x x} & 0 \\
0 & \mathbf{D}_{y y}^{*}
\end{array}\right)\right) .
$$

Здесъ $\mathbf{D}_{x x}$ - первая компонента матрицы супердифбузии $\mathbf{D}$ из теоремъ 5, a $\mathbf{D}_{y y}^{*}$ - последняя компонента матрицы диффузии $\mathbf{D}^{*}$ из теоремы 1.

(б) При этом имеет место слабый принцип инвариантности, т.е. процесс

$$
\left(\frac{x(s T)}{\sqrt{T \log T}}, \frac{y(s T)}{\sqrt{T}}\right), \quad 0<s<1,
$$

сходится при $T \rightarrow \infty$ к броуновскому движению с нулевым средним и с ковариационной матрищей $\left(\begin{array}{cc}\mathbf{D}_{x x} & 0 \\ 0 & \mathbf{D}_{y y}^{*}\end{array}\right)$.

Заметим, что предположение о справедливости дискретного варианта теоремы 7 (а) было высказано в [2; с. 70].

Далее мы модифицируем предложение 2.1 в случае, когда все коридоры параллельны.

ПРЕДЛОЖЕНИЕ 2.2. В условиях теоремы 6 предположим, что все коридоры параллельны оси $x$. Тогда матрица дифбузии $\mathbf{D}_{\varepsilon}$ удовлетворяет соотношениям

$$
\begin{aligned}
& \left(\mathbf{D}_{\varepsilon}\right)_{x x}=|\log \varepsilon| \mathbf{D}_{x x}+\mathscr{O}(1), \\
& \left(\mathbf{D}_{\varepsilon}\right)_{x y}=\mathbf{D}_{x y}^{*}+o(1), \\
& \left(\mathbf{D}_{\varepsilon}\right)_{y y}=\mathbf{D}_{y y}^{*}+o(1)
\end{aligned}
$$

Из предложения 2.2 следует, что у матрицы диффузии $\mathbf{D}_{\varepsilon}$ два собственных значения: большее имеет порядок $|\log \varepsilon| \mathbf{D}_{x x}$, а меньшее - порядок $\mathbf{D}_{y y}^{*}$, и соответствующие собственные векторы $\mathscr{O}\left(|\log \varepsilon|^{-1}\right)$-близки к осям координат.

ПРЕДЛОЖЕНИЕ 2.3. В условиях теоремы 6 предположим, что все коридоры параллельны оси $x$. Пусть одновременно $t \rightarrow \infty u \varepsilon \rightarrow 0$. Тогда

$$
\left(\frac{x(t)}{\sqrt{t \log \min \left\{t, \varepsilon^{-1}\right\}}}, \frac{y(t)}{\sqrt{t}}\right) \Rightarrow \mathscr{N}\left(0,\left(\begin{array}{cc}
\mathbf{D}_{x x} & 0 \\
0 & \mathbf{D}_{y y}^{*}
\end{array}\right)\right) .
$$

Смежные вопросы. Имеется ряд дополнительных интересных свойств газа Лоренца под действием малого внешнего поля с гауссовым термостатом. Для случая конечного горизонта они были выведены в [1], [10]; на случай бесконечного горизонта рассуждения переносятся без изменений. Перечислим здесь все эти результаты, отсылая за доказательствами к [1]. 
Во-первых, пусть $\lambda_{\varepsilon}^{-}<0<\lambda_{\varepsilon}^{+}$обозначают положительный и отрицательный показатели Ляпунова потока $\Phi_{\varepsilon}^{t}$ (у него есть также один нулевой показатель Ляпунова). Их сумма $\lambda_{\varepsilon}^{+}+\lambda_{\varepsilon}^{-}$представляет собой среднюю скорость сжатия объема под действием потока $\Phi^{t}$. Точнее, если $\dot{X}=\mathbf{V}(X)$ - уравнения движения ( $X$ обозначает точку в фазовом пространстве потока, $\dot{X}-$ ее производную по времени, а $\mathbf{V}(X)$ - поле векторов скорости), то

$$
\lambda_{\varepsilon}^{+}+\lambda_{\varepsilon}^{-}=\mu_{\varepsilon}\left(\nabla_{X} \cdot \mathbf{V}(X)\right),
$$

где $\nabla_{X} \cdot \mathbf{V}(X)$ - дивергенция $\mathbf{V}$ (см. [1; с. 572]). Особенностью наших уравнений движения (1.5) является то, что

$$
\nabla_{X} \cdot \mathbf{V}(X)=-\langle\mathbf{E}, \mathbf{v}\rangle
$$

в обозначениях (1.5). Поэтому

$$
\lambda_{\varepsilon}^{+}+\lambda_{\varepsilon}^{-}=-\langle\mathbf{E}, \mathbf{J}\rangle .
$$

В контексте теоремы 2 (т. е. для конечного горизонта) получаем

$$
\lambda_{\varepsilon}^{+}+\lambda_{\varepsilon}^{-}=-\frac{1}{2} \mathbf{E}^{T} \mathbf{D}^{*} \mathbf{E}+o\left(\|\mathbf{E}\|^{2}\right),
$$

а в контексте теоремы 6 (бесконечный горизонт)

$$
\lambda_{\varepsilon}^{+}+\lambda_{\varepsilon}^{-}=-\frac{1}{2} \mathbf{E}^{T} \mathbf{D E}|\log \|\mathbf{E}\||+\mathscr{O}\left(\|\mathbf{E}\|^{2}\right) .
$$

Аналогичным образом, пусть $\hat{\lambda}_{\varepsilon}^{-}<0<\hat{\lambda}_{\varepsilon}^{+}$обозначают показатели Ляпунова отображения столкновений $\mathscr{F}_{\varepsilon}$. Их сумма представляет собой среднюю скорость сжатия объема этим отображением, т. е.

$$
\hat{\lambda}_{\varepsilon}^{+}+\hat{\lambda}_{\varepsilon}^{-}=\nu_{\varepsilon}(\log g),
$$

где $g=d \mathscr{F}_{\varepsilon}^{-1} \nu_{0} / d \nu_{0}-$ якобиан отображения $\mathscr{F}_{\varepsilon}$ относительно биллиардной инвариантной меры $\nu_{0}$. Снова непосредственное вычисление показывает, что $g=e^{-\left\langle\mathbf{E}, \boldsymbol{\Delta}_{\varepsilon}\right\rangle} ;$ отсюда

$$
\hat{\lambda}_{\varepsilon}^{+}+\hat{\lambda}_{\varepsilon}^{-}=-\bar{\tau}_{\varepsilon}\langle\mathbf{E}, \mathbf{J}\rangle .
$$

Таким образом получаем

$$
\hat{\lambda}_{\varepsilon}^{+}+\hat{\lambda}_{\varepsilon}^{-}=-\frac{1}{2} \bar{\tau}_{0} \mathbf{E}^{T} \mathbf{D}^{*} \mathbf{E}+o\left(\|\mathbf{E}\|^{2}\right)
$$

для случая конечного горизонта и

$$
\hat{\lambda}_{\varepsilon}^{+}+\hat{\lambda}_{\varepsilon}^{-}=-\frac{1}{2} \bar{\tau}_{0} \mathbf{E}^{T} \mathbf{D E}|\log \|\mathbf{E}\||+\mathscr{O}\left(\|\mathbf{E}\|^{2}\right)
$$

для бесконечного горизонта. Мы использовали тот факт, что $\bar{\tau}_{\varepsilon}=\bar{\tau}_{0}+\mathscr{O}\left(\varepsilon^{a}\right)$ для некоторого $a>0$, что будет доказано в предложении 3.1.

Формулы (2.8)-(2.11) позволяют оценить фрактальную (информационную) размерность (обозначаемую через $\mathrm{HD}$ ) инвариантных мер $\mu_{\varepsilon}$ и $\nu_{\varepsilon}$. Во-первых, $\mathrm{HD}\left(\mu_{\varepsilon}\right)=\mathrm{HD}\left(\nu_{\varepsilon}\right)+1$, и по формуле Янг [22]

$$
\operatorname{HD}\left(\nu_{\varepsilon}\right)=h_{\nu_{\varepsilon}}\left(\mathscr{F}_{\varepsilon}\right)\left(\frac{1}{\hat{\lambda}_{\varepsilon}^{+}}-\frac{1}{\hat{\lambda}_{\varepsilon}^{-}}\right),
$$


где $h_{\nu_{\varepsilon}}\left(\mathscr{F}_{\varepsilon}\right)$ - энтропия Колмогорова-Синая отображения $\mathscr{F}_{\varepsilon}$. По формуле Песина $h_{\nu_{\varepsilon}}\left(\mathscr{F}_{\varepsilon}\right)=\hat{\lambda}_{\varepsilon}^{+}$. Отсюда следует, что

$$
\operatorname{HD}\left(\mu_{\varepsilon}\right)=2-\frac{\lambda_{\varepsilon}^{+}}{\lambda_{\varepsilon}^{-}}=3-\frac{\lambda_{\varepsilon}^{+}+\lambda_{\varepsilon}^{-}}{\lambda_{\varepsilon}^{-}} .
$$

Поэтому

$$
\mathrm{HD}\left(\mu_{\varepsilon}\right)=3-\frac{\bar{\tau}_{0}}{2 h_{0}} \mathbf{E}^{T} \mathbf{D}^{*} \mathbf{E}+o\left(\|\mathbf{E}\|^{2}\right)
$$

в случае конечного горизонта и

$$
\mathrm{HD}\left(\mu_{\varepsilon}\right)=3-\frac{\bar{\tau}_{0}}{2 h_{0}} \mathbf{E}^{T} \mathbf{D E}|\log \|\mathbf{E}\||+o\left(\|\mathbf{E}\|^{2}|\log \|\mathbf{E}\||\right)
$$

в случае бесконечного горизонта. Здесь $h_{0}=h_{\nu_{0}}\left(\mathscr{F}_{0}\right)$ обозначает энтропию Колмогорова-Синая биллиардного отображения $\mathscr{F} 0$, и мы воспользовались непрерывностью функции $h_{\nu_{\varepsilon}}\left(\mathscr{F}_{\varepsilon}\right)=\hat{\lambda}_{\varepsilon}^{+}$в точке $\varepsilon=0$; см. [1]. Заметим также, что $h_{0} / \bar{\tau}_{0}=h_{\mu_{0}}\left(\Phi^{t}\right)$ - энтропия Колмогорова-Синая биллиардного потока $\Phi^{t}$.

Из наших формул $(2.12),(2.13)$ следует, что мера $\mu_{\varepsilon}$ сингулярна относительно объема Лебега при малых $\varepsilon \neq 0$, и это же верно для $\nu_{\varepsilon}$. С другой стороны, носитель меры $\mu_{\varepsilon}$ - все фазовое пространство $\Omega$, а носитель меры $\nu_{\varepsilon}-$ все пространство $\mathscr{M}$, так как любое открытое множество имеет положительную меру (теорема 6); такая ситуация обычна для SRB-мер, полученных возмущениями гладкой меры, т.е. для неравновесных стационарных установившихся состояний, близких к равновесию [1], [10].

Это завершает формулировку наших результатов.

Структура работы. Работа организована следующим образом. В разделе 3 мы переходим к дискретному времени, т. е. переформулируем наши основные теоремы в терминах соответствующих отображений столкновений. Затем мы начинаем доказательство теоремы 6 , которое занимает семь разделов. В помощь читателю в разделе 4 дается схема наших рассуждений, а технические вычисления отнесены в следующие шесть разделов. Затем в разделе 5 проводится определенная подготовка: описание геометрии траекторий в бесконечных коридорах и получающегося строения пространства столкновений $\mathscr{M}$. В разделах 6-7 дается обзор стандартных методов изучения гиперболических систем с сингулярностями. В разделах 8-10 выполняется основная часть технического анализа.

Далее в разделе 11 мы доказываем относительно более простые теоремы 5 и 7. В разделе 12 содержатся некоторые технические оценки, нужные для раздела 11, и завершаются доказательства предложений 2.1-2.3.

\section{3. Переход к дискретному времени}

Стандартным приемом при изучении динамических систем с непрерывным временем (потоков) является их перевод на дискретное время с помощью отображения последования на подходящим образом построенном сечении в фазовом пространстве. 
3.1. Газ Лоренца без внешних сил. Начнем с относительно более простого свободного газа Лоренца. Как обычно, представим динамику $\Phi^{t}: \Omega \rightarrow \Omega$ с непрерывным временем потоком надстройки над отображением столкновений $\mathscr{F}: \mathscr{M} \rightarrow \mathscr{M}$ под функцией потолка $\tau$. Теперь наши теоремы 5 и 7 можно следующим образом переформулировать для полученного процесса с дискретным временем.

ТеОрема 8. Пусть $\widetilde{\mathbf{q}}_{n}=\left(x_{n}, y_{n}\right)$ - положение в момент $n$-го столкновения частицы в газе Лоренца с бесконечным горизонтом (без внешних полей).

(а) Если есть два непараллельных коридора, то выполняется следующий слабый принчип инвариантности: процесс

$$
\frac{\widetilde{\mathbf{q}}_{s n}}{\sqrt{n \log n}}, \quad 0<s<1
$$

сходится при $n \rightarrow \infty \kappa$ броуновскому движению с нулевым средним и с ковариационной матрицей $\widehat{\mathbf{D}}$, заданной в (2.1).

(б) Если все бесконечные коридоры параллельны (и система координат выбрана так, что осъ $x$ параллельна коридорам), то выполняется следующий слабый принцип инвариантности: процесс

$$
\left(\frac{x_{s n}}{\sqrt{n \log n}}, \frac{y_{s n}}{\sqrt{n}}\right), \quad 0<s<1,
$$

сходится при $n \rightarrow \infty \kappa$ броуновскому движению с нулевым средним и с ковариационной матрицей $\left(\begin{array}{cc}\widehat{\mathbf{D}}_{x x} & 0 \\ 0 & \widehat{\mathbf{D}}_{y y}^{*}\end{array}\right)$, где $\widehat{\mathbf{D}}^{*}=\bar{\tau}_{0} \mathbf{D}^{*} ; c p$. (1.3).

Здесь, как обычно, $\widetilde{\mathbf{q}}_{s n}-$ непрерывная функция от $s$ такая, что $\widetilde{\mathbf{q}}_{s n}=\widetilde{\mathbf{q}}_{m}$, если $s n=m$ - целое число, и полученная линейной интерполяцией между целыми числами.

Теперь можно вывести теоремы 5 и 7 из теоремы 8. Общие результаты, подобные [23], не применимы потому, что наши функции $\boldsymbol{\Delta}$ и $\tau$ неограничены и быстро растут (собственно, их вторые моменты расходятся: $\nu_{0}\left(\tau^{2}\right)=$ $\left.\nu_{0}\left(\|\boldsymbol{\Delta}\|^{2}\right)=\infty\right)$; значит, нужно дать рассуждение, специально приспособленное к задаче.

В нашей модели функция $\|\boldsymbol{\Delta}\|=\tau$ принимает большие значения, когда траектория входит в бесконечный коридор для длительного свободного пробега без столкновений. Известно (см. [7; §4.10] и [2]), что для всех $A>1$

$$
\nu_{0}(\tau>A)=\mathscr{O}\left(A^{-2}\right) .
$$

Теперь для заданных $T>0$ и $s \in(0,1)$ пусть $m_{1}=m_{1}(s, T)=\left[s T / \bar{\tau}_{0}\right]$ и пусть $m_{2}=m_{2}(s, T, X)$ - такое целое число, что $t_{m_{2}}(X) \leqslant s T<t_{m_{2}+1}(X)$, где $t_{m}(X)$ обозначает время $m$-го столкновения. Из оценки (3.1) следует, что

$$
\nu_{0}\left(\max _{m \leqslant 2 T / \bar{\tau}_{0}}\left|t_{m+1}-t_{m}\right| \leqslant \sqrt{T} \log \log T\right) \rightarrow 1
$$

при $T \rightarrow \infty$. Заметим, что $\left\|\widetilde{\mathbf{q}}\left(t^{\prime}\right)-\widetilde{\mathbf{q}}\left(t^{\prime \prime}\right)\right\| \leqslant\left|t^{\prime}-t^{\prime \prime}\right|$, так как $\|\mathbf{v}(t)\|=1$. Итак, достаточно показать, что

$$
\frac{\max _{0<s<1}\left|\widetilde{\mathbf{q}}_{m_{1}}-\widetilde{\mathbf{q}}_{m_{2}}\right|}{\sqrt{T \log T}} \rightarrow 0
$$


по вероятности. Из эргодической теоремы следует, что $t_{m}(X) / m \rightarrow \bar{\tau}_{0}$ почти всюду при $m \rightarrow \infty$. Отсюда для заданных $\varepsilon_{1}, \varepsilon_{2}>0$ получаем, что для достаточно больших $T$ и для всех $s \in(0,1)$

$$
\nu_{0}\left(t_{m_{1}-\varepsilon_{1} T}<s T \text { и } t_{m_{1}+\varepsilon_{1} T}>s T\right) \geqslant 1-\varepsilon_{2} .
$$

Ясно, что из (3.2) и (3.3) следует, что

$$
\nu_{0}\left(\left|m_{2}-m_{1}\right|<\varepsilon_{1} T\right)>1-2 \varepsilon_{2} .
$$

С другой стороны, в силу теоремы $8\left(\right.$ a) процесс $\widetilde{\mathbf{q}}_{s T} / \sqrt{T \log T}$ является предкомпактным, т. е. для любого $\varepsilon_{1}>0$ существует $\varepsilon_{2}>0$ такое, что если $\left|m_{1}-m_{2}\right|<$ $\varepsilon_{2} T$, то

$$
\nu_{0}\left(\left|\widetilde{\mathbf{q}}_{m_{1}}-\widetilde{\mathbf{q}}_{m_{2}}\right|<\varepsilon_{1} \sqrt{T \log T}\right) \geqslant 1-\varepsilon_{1} .
$$

Отсюда следует слабый принцип инвариантности в теореме 5 (в), а центральная предельная теорема в части (а) немедленно следует из (в). Вывод теоремы 7 из теоремы 8(б) очень похожий, и мы его опускаем.

Теорема 8, в свою очередь, является распространением недавних результатов Д. Саса и Т. Варью [2] (теорема 4); она доказывается в разделе 11.

3.2. Газ Лоренца под действием внешнего поля. Теперь обратимся к нашей основной цели - газу Лоренца под действием малого внешнего поля $\mathbf{E}$. Напомним, что мы фиксируем направление $\mathbf{E}$ и рассматриваем $\varepsilon=\|\mathbf{E}\|$ в качестве малого параметра модели. Мы снова представим динамику с непрерывным временем $\Phi_{\varepsilon}^{t}: \Omega \rightarrow \Omega$ потоком надстройки над отображением столкновений $\mathscr{F}_{\varepsilon}: \mathscr{M} \rightarrow \mathscr{M}$ под функцией потолка

$$
\tau_{\varepsilon}(X)=\min \left\{t>0: \Phi_{\varepsilon}^{t}(X) \in \mathscr{M}\right\} .
$$

Теперь теорему 6 можно следующим образом переформулировать для дискретного времени.

ТЕОрема 9. Пусть $\widetilde{\mathbf{q}}_{n}$ - положение в момент $n$-го столкновения частииы в газе Лоренца с бесконечным горизонтом и с малым внешним полем $\mathbf{E}$, спаренным с гауссовым термостатом (1.5). Предположим, что $\mathbf{E}$ не параллельно ни одному из бесконечных коридоров.

(а) Для достаточно малого в отображение столкновений $\mathscr{F}_{\varepsilon}$ гиперболично; оно сохраняет (SRB-) меру Синая-Рюэля-Боуэна (установившееся состояние) $\nu_{\varepsilon}$, которая эргодична, перемешивающая и положительна на открытых множествах. Электрический ток с дискретным временем

$$
\widehat{\mathbf{J}}=\lim _{n \rightarrow \infty} \frac{\widetilde{\mathbf{q}}_{n}}{n}=\nu_{\varepsilon}\left(\boldsymbol{\Delta}_{\varepsilon}\right)
$$

корректно определен.

(б) Ток $\widehat{\mathbf{J}}$ удовлетворяет равенству

$$
\widehat{\mathbf{J}}=\frac{1}{2}|\log \varepsilon| \widehat{\mathbf{D}} \mathbf{E}+\delta \widehat{\mathbf{J}},
$$

где

$$
\widehat{\mathbf{D}}=\bar{\tau}_{0} \mathbf{D},
$$


$\bar{\tau}_{0}$ задано в (1.4), а D - матрица из теоремы 5. Остаточный член $\delta \widehat{\mathbf{J}}$ в равенстве (3.5) ограничен следующим образом:

$$
\|\delta \widehat{\mathbf{J}}\| \leqslant \widehat{A}_{\mathbf{u}} \varepsilon
$$

где $\widehat{A}_{\mathbf{u}}>0$ зависит только от направления $\mathbf{u}=\mathbf{E} /\|\mathbf{E}\|$ поля.

(в) Имеет место следующая слабая сходимость при $n \rightarrow \infty$ :

$$
\frac{\widetilde{\mathbf{q}}_{n}-\widehat{\mathbf{J}}_{n}}{\sqrt{n}} \Rightarrow \mathscr{N}\left(0, \widehat{\mathbf{D}}_{\varepsilon}\right),
$$

где $\widehat{\mathbf{D}}_{\varepsilon}-$ матрица диффузии для дискретного времени.

(г) Матрица $\widehat{\mathbf{D}}_{\varepsilon}$ удовлетворяет равенству

$$
\widehat{\mathbf{D}}_{\varepsilon}=|\log \varepsilon| \widehat{\mathbf{D}}+\delta \widehat{\mathbf{D}},
$$

где $\|\delta \widehat{\mathbf{D}}\| \leqslant \widehat{B}_{\mathbf{u}}\left(\widehat{B}_{\mathbf{u}}-\right.$ константа, зависящая только от $\left.\mathbf{u}\right)$.

Чтобы вывести нашу основную теорему 6 из теоремы 9, нам потребуется следующая техническая оценка.

ПРЕДЛОЖЕНИЕ 3.1. Средний свободный пробег $\bar{\tau}_{\varepsilon}=\nu_{\varepsilon}\left(\tau_{\varepsilon}\right)$ является гёльдеровски непрерывной функиией в точке $\varepsilon=0$, m.e.

$$
\left|\bar{\tau}_{\varepsilon}-\bar{\tau}_{0}\right| \leqslant \widehat{C}_{\mathbf{u}} \varepsilon^{a}
$$

для некоторых констант $\widehat{C}_{\mathbf{u}}, a>0$, где $\widehat{C}_{\mathbf{u}}$ зависит толъко от $\mathbf{u}$.

Теперь теорему 6 несложно вывести из теоремы 9. Заметим только, что центральная предельная теорема (2.5) для непрерывного времени следует из соответствующего ее аналога (3.8) для дискретного времени согласно общим результатам (ср. [23] или [7; теорема 7.68]), поскольку (как мы покажем в разделе 5 ) функции $\boldsymbol{\Delta}_{\varepsilon}$ и $\tau_{\varepsilon}$ ограничены и регулярны (точнее, они динамически гёльдеровски непрерывны, см. определение в разделе 7).

Остается доказать теорему 9, и это делается в следующих семи разделах.

\section{4. План доказательства теоремы 9}

Здесь мы очертим структуру наших рассуждений, оставляя технические вычисления для следующих шести разделов.

Отображение $\mathscr{F}_{\varepsilon}$ является малым возмущением биллиардного отображения $\mathscr{F}$, которое известно своими сильными гиперболическими и эргодическими свойствами. В случае конечного горизонта теория возмущений была развита в [1], [10], [12], [13] для доказательства существования SRB-меры $\nu_{\varepsilon}$ и ее эргодических и статистических свойств, включая экспоненциальное убывание корреляций.

В нашем случае имеется более серьезное различие между $\mathscr{F} \varepsilon$ и $\mathscr{F}$. Второе отображение обладает счетным числом линий разрыва, которые накапливаются вблизи неподвижных точек $X \in \mathscr{M}: \mathscr{F}(X)=X$ (соответствующих границам бесконечных коридоров; ср. (2.1)). Линии разрыва вблизи неподвижных 
точек образуют характерные участки (называемых ячейками; см., например, $[7 ; \S 4.10])$, которые определяют глобальные свойства отображения $\mathscr{F}$.

Наше отображение $\mathscr{F}_{\varepsilon}$, с другой стороны, имеет только конечное число линий разрыва, и строение соответствующих ячеек совершенно иное. Хотя наша ситуация проще из-за конечности числа линий разрыва, наши ячейки нужно тщательно проанализировать для того, чтобы вывести свойства $\mathscr{F}$, подобные свойствам $\mathscr{F}$. Этот анализ проводится в разделе 5 ; там мы покажем, что $\tau_{\varepsilon}$ и $\left\|\boldsymbol{\Delta}_{\varepsilon}\right\|$ не превосходят const $\cdot \varepsilon^{-1 / 2}$.

Теперь рассуждение о возмущении, развитое в [1], [10], [12], [13], можно снова использовать для построения единственной SRB-меры $\nu_{\varepsilon}$ и для доказательства ее эргодических и статистических свойств; это делается в разделах 6,7 .

Одно из основных свойств SRB-меры $\nu_{\varepsilon}$ состоит в том, что она является слабым пределом $\nu_{\varepsilon}=\lim _{n \rightarrow \infty} \mathscr{F}_{\varepsilon}^{n} \nu_{0}$. Кроме того, для любой гладкой функции $f$ на $\mathscr{M}$ имеется формула типа Кавасаки:

$$
\begin{aligned}
\nu_{\varepsilon}(f) & =\lim _{n \rightarrow \infty} \nu_{0}\left(f \circ \mathscr{F}_{\varepsilon}^{n}\right)=\nu_{0}(f)+\lim _{n \rightarrow \infty} \sum_{k=1}^{n} \nu_{0}\left[\left(f \circ \mathscr{F}_{\varepsilon}^{k}\right)-\left(f \circ \mathscr{F}_{\varepsilon}^{k-1}\right)\right] \\
& =\nu_{0}(f)+\sum_{k=1}^{\infty} \nu_{0}\left[\left(f \circ \mathscr{F}_{\varepsilon}^{k}\right)(1-g)\right],
\end{aligned}
$$

где $g=\frac{d \mathscr{F}_{\varepsilon}^{-1} \nu_{0}}{d \nu_{0}}=e^{-\left\langle\mathbf{E}, \boldsymbol{\Delta}_{\varepsilon}\right\rangle}-$ якобиан отображения $\mathscr{F}_{\varepsilon}$ относительно биллиардной инвариантной меры $\nu_{0}$. Мы покажем, что ряд в (4.1) сходится экспоненциально быстро и равномерно по $\varepsilon$. Полезно заметить, что $\nu_{0}(1-g)=0$, так как $g$ - плотность вероятностной меры.

Снова для удобства выберем систему координат так, чтобы направление поля $\mathbf{E}$ совпадало с положительным направлением оси $x$; тогда $\mathbf{E}=(\varepsilon, 0)$. Обозначая компоненты вектора смещения через $\boldsymbol{\Delta}_{\varepsilon}=\left(\Delta_{\varepsilon, x}, \Delta_{\varepsilon, y}\right)$, получаем

$$
g=e^{-\left\langle\mathbf{E}, \boldsymbol{\Delta}_{\varepsilon}\right\rangle}=e^{-\varepsilon \Delta_{\varepsilon, x}} .
$$

Согласно (3.4), нам нужно применить (4.1) к функциям $\Delta_{\varepsilon, x}$ и $\Delta_{\varepsilon, y}$, которые являются лишь кусочно гладкими и не являются равномерно ограниченными (точнее, $\sup _{\mathscr{M}}\left\|\boldsymbol{\Delta}_{\varepsilon}\right\| \sim \varepsilon^{-1 / 2}$ ). Все же формула Кавасаки (4.1) применима, и ряд сходится равномерно по $\varepsilon$; это будет доказано в разделе 8.

Далее мы используем инвариантность меры $\nu_{\varepsilon}$, чтобы записать (имея в виду $\Delta_{\varepsilon}=\Delta_{\varepsilon, x}$ или $\left.\Delta_{\varepsilon, y}\right)$

$$
\begin{gathered}
\nu_{\varepsilon}\left(\Delta_{\varepsilon}\right)=\frac{1}{2}\left(\nu_{\varepsilon}\left(\Delta_{\varepsilon}\right)+\nu_{\varepsilon}\left(\Delta_{\varepsilon} \circ \mathscr{F}_{\varepsilon}^{-1}\right)\right)=\frac{1}{2}\left(\nu_{0}\left(\Delta_{\varepsilon}\right)+\nu_{0}\left(\Delta_{\varepsilon} \circ \mathscr{F}_{\varepsilon}^{-1}\right)\right) \\
+\sum_{k=1}^{\infty} \nu_{0}\left[\left(\Delta_{\varepsilon} \circ \mathscr{F}_{\varepsilon}^{k}\right)(1-g)\right]+\frac{1}{2} \nu_{0}\left[\Delta_{\varepsilon}(1-g)\right] .
\end{gathered}
$$

Благодаря тому, что наша динамика допускает обращение времени, первый член обращается в нуль, т. е. $\nu_{0}\left(\Delta_{\varepsilon}\right)+\nu_{0}\left(\Delta_{\varepsilon} \circ \mathscr{F}_{\varepsilon}^{-1}\right)=0$, см. также [1; с. 585]. Далее, воспользуемся разложением Тейлора

$$
1-g=\varepsilon \Delta_{\varepsilon, x}+\varepsilon \mathscr{R}_{\varepsilon},
$$


где $\mathscr{R}_{\varepsilon}$ обозначает остаточный член. Мы покажем в разделе 8 , что $\mathscr{R}_{\varepsilon}=\mathscr{O}(1)$ равномерно по $\varepsilon$. Кроме того, $\mathscr{R}_{\varepsilon}$ имеет ту же степень регулярности, что и сама функция $\Delta_{\varepsilon, x}$, а $\mathscr{R}_{\varepsilon} \rightarrow 0$ поточечно при $\varepsilon \rightarrow 0$. Таким образом, можно будет легко подавить вклад члена $\mathscr{R}_{\varepsilon}$. Теперь

$$
\sum_{n=1}^{\infty} \nu_{0}\left[\left(\Delta_{\varepsilon} \circ \mathscr{F}_{\varepsilon}^{n}\right)(1-g)\right]=\varepsilon \sum_{n=1}^{\infty} \nu_{0}\left[\left(\Delta_{\varepsilon} \circ \mathscr{F}_{\varepsilon}^{n}\right)\left(\Delta_{\varepsilon, x}+\mathscr{R}_{\varepsilon}\right)\right] .
$$

В правой части стоит сумма похожих на корреляции членов, которая, как мы покажем, равномерно ограничена; значит, левая часть является $\mathscr{O}(\varepsilon)$. Равномерные оценки корреляций составляют наши основные технические результаты; они приводятся в разделе 9 .

Теперь мы видим, что основной вклад в ток $\widehat{\mathbf{J}}$ дает последний член в (4.3), который равен

$$
\nu_{0}\left[\Delta_{\varepsilon}(1-g)\right]=\varepsilon \nu_{0}\left(\Delta_{\varepsilon} \Delta_{\varepsilon, x}\right)+\varepsilon \nu_{0}\left(\Delta_{\varepsilon} \mathscr{R}_{\varepsilon}\right)
$$

Последним членом можно пренебречь, так как мы покажем, что

$$
\nu_{0}\left(\Delta_{\varepsilon, x} \mathscr{R}_{\varepsilon}\right)=\nu_{0}\left(\Delta_{\varepsilon, y} \mathscr{R}_{\varepsilon}\right)=\mathscr{O}(\sqrt{\varepsilon})
$$

Наконец, мы покажем непосредственным вычислением (см. раздел 10), что

$$
\nu_{0}\left(\Delta_{\varepsilon, x}^{2}\right)=\widehat{\mathbf{D}}_{x x}|\log \varepsilon|+\mathscr{O}(1)
$$

и

$$
\nu_{0}\left(\Delta_{\varepsilon, x} \Delta_{\varepsilon, y}\right)=\widehat{\mathbf{D}}_{x y}|\log \varepsilon|+\mathscr{O}(1),
$$

где $\widehat{\mathbf{D}}_{x x}$ и $\widehat{\mathbf{D}}_{x y}$ - соответствующие компоненты матрицы супердиффузии $\widehat{\mathbf{D}}$, заданной в (2.1). Это завершит наше доказательство соотношений (3.5)-(3.7).

Обратимся к (3.8), (3.9). Сходимость к нормальному закону $\mathscr{N}\left(\mathbf{0}, \widehat{\mathbf{D}}_{\varepsilon}\right)$ - это просто центральная предельная теорема, доказываемая стандартными рассуждениями [21], [12] (которые применимы потому, что функция $\boldsymbol{\Delta}_{\varepsilon}$ ограничена и гёльдеровски непрерывна). Матрица ковариации $\widehat{\mathbf{D}}_{\varepsilon}$ дается суммой корреляций:

$$
\widehat{\mathbf{D}}_{\varepsilon}=\sum_{n=-\infty}^{\infty}\left(\nu_{\varepsilon}\left[\left(\boldsymbol{\Delta}_{\varepsilon} \circ \mathscr{F}_{\varepsilon}^{n}\right) \otimes \boldsymbol{\Delta}_{\varepsilon}\right]-\nu_{\varepsilon}\left(\boldsymbol{\Delta}_{\varepsilon}\right) \otimes \nu_{\varepsilon}\left(\boldsymbol{\Delta}_{\varepsilon}\right)\right) .
$$

Мы покажем, что этот ряд, за исключением его центрального члена $n=0$, сходится экспоненциально быстро и равномерно по $\varepsilon$; мы также покажем, что $\nu_{\varepsilon}\left(\boldsymbol{\Delta}_{\varepsilon}\right)=\mathscr{O}(1)$ равномерно по $\varepsilon$. Отсюда будет следовать соотношение

$$
\widehat{\mathbf{D}}_{\varepsilon}=\nu_{\varepsilon}\left(\boldsymbol{\Delta}_{\varepsilon} \otimes \boldsymbol{\Delta}_{\varepsilon}\right)+\mathscr{O}(1) .
$$

Далее мы проверим, что

$$
\nu_{\varepsilon}\left(\boldsymbol{\Delta}_{\varepsilon} \otimes \boldsymbol{\Delta}_{\varepsilon}\right)=\nu_{0}\left(\boldsymbol{\Delta}_{\varepsilon} \otimes \boldsymbol{\Delta}_{\varepsilon}\right)+\mathscr{O}(1)
$$

(заметим, что из этого выражения вместе с (4.3)-(4.5) следует соотношение Эйнштейна между электрической проводимостью и матрицей диффузии). 
С этой целью мы применим формулу типа Кавасаки (4.1) к каждой компоненте матрицы

$$
\boldsymbol{\Delta}_{\varepsilon} \otimes \boldsymbol{\Delta}_{\varepsilon}=\left[\begin{array}{cc}
\Delta_{\varepsilon, x}^{2} & \Delta_{\varepsilon, x} \Delta_{\varepsilon, y} \\
\Delta_{\varepsilon, x} \Delta_{\varepsilon, y} & \Delta_{\varepsilon, y}^{2}
\end{array}\right] .
$$

Они рассматриваются похожим образом, и мы приводим только формулы для $\Delta_{\varepsilon, x}^{2}$ :

$$
\begin{aligned}
\nu_{\varepsilon}\left(\Delta_{\varepsilon, x}^{2}\right) & =\nu_{0}\left(\Delta_{\varepsilon, x}^{2}\right)+\sum_{k=1}^{\infty} \nu_{0}\left[\left(\Delta_{\varepsilon, x}^{2} \circ \mathscr{F}_{\varepsilon}^{k}\right)(1-g)\right] \\
& =\nu_{0}\left(\Delta_{\varepsilon, x}^{2}\right)+\varepsilon \sum_{n=1}^{\infty} \nu_{0}\left[\left(\Delta_{\varepsilon, x}^{2} \circ \mathscr{F}_{\varepsilon}^{n}\right)\left(\Delta_{\varepsilon, x}+\mathscr{R}_{\varepsilon}\right)\right] .
\end{aligned}
$$

Мы докажем, что ряд не превосходит $\varepsilon^{-a}$ для некоторого $a<1$; отсюда получим (4.10). Далее, компоненты матрицы $\nu_{0}\left(\boldsymbol{\Delta}_{\varepsilon} \otimes \boldsymbol{\Delta}_{\varepsilon}\right)$ были подсчитаны в (4.7), (4.8); таким образом приходим к (3.9). Это завершит доказательство теоремы 9 .

Наконец, для доказательства предложения 3.1 нужно оценить $\left|\nu_{\varepsilon}\left(\tau_{\varepsilon}\right)-\nu_{0}(\tau)\right|$. Начнем с применения формулы Кавасаки (4.1) к $\tau_{\varepsilon}$ :

$$
\nu_{\varepsilon}\left(\tau_{\varepsilon}\right)=\nu_{0}\left(\tau_{\varepsilon}\right)+\varepsilon \sum_{k=1}^{\infty} \nu_{0}\left[\left(\tau_{\varepsilon} \circ \mathscr{F}_{\varepsilon}^{k}\right)\left(\Delta_{\varepsilon, x}+\mathscr{R}_{\varepsilon}\right)\right],
$$

где ряд, как будет показано, сходится экспоненциально и равномерно по $\varepsilon$. Таким образом, достаточно оценить $\nu_{0}\left(\left|\tau_{\varepsilon}-\tau\right|\right)$, и мы покажем, что

$$
\nu_{0}\left(\left|\tau_{\varepsilon}-\tau\right|\right)=\mathscr{O}\left(\varepsilon^{a}\right)
$$

для некоторого $a>0$ (это будет сделано прямой геометрической оценкой в разделе 10).

\section{5. Геометрический анализ}

Уравнения движения (1.5) имеют явное решение: так как $\|\mathbf{v}\|=1$, можно положить $\mathbf{v}=(\cos \theta, \sin \theta)$, тогда (1.5) принимает вид

$$
\dot{x}=\cos \theta, \quad \dot{y}=\sin \theta, \quad \dot{\theta}=-\varepsilon \sin \theta
$$

(где $\dot{x}=d x / d t$ и т. п.), и решение дается формулами

$$
\begin{aligned}
& x=x_{0}+\frac{1}{\varepsilon} \log \frac{\sin \theta_{0}}{\sin \theta}, \\
& y=y_{0}+\frac{\theta_{0}-\theta}{\varepsilon}, \\
& \theta=2 \arctan \left(c_{0} e^{-\varepsilon t}\right),
\end{aligned}
$$

где $c_{0}=\tan \left(\theta_{0} / 2\right)$ и $\left(x_{0}, y_{0}, \theta_{0}\right)$ обозначают начальные значения. Когда частица сталкивается с рассеивателем, $\theta$ изменяется согласно правилу зеркального отражения. 
Последующие результаты можно вывести из (5.2) прямыми аналитическими вычислениями, но мы приводим более геометрическое рассуждение, использующее преобразование Войтковского [24]. Оно основано на замене переменных, которая преобразует изогнутые траектории (5.2) в прямые. Пусть $z=x+i y-$ комплексная переменная, заменяющая $x$ и $y$. Преобразуем $\widetilde{\mathscr{D}}$ по правилу

$$
z \mapsto w=T(z)=\int e^{-\varepsilon z} d z=\frac{1}{\varepsilon}\left[e^{\varepsilon z}-1\right]
$$

(член -1 добавлен, чтобы начало координат оставалось на месте). В вещественных переменных, если обозначить $w=u+i v$, это преобразование действует как $T(x, y)=(u, v)$, где

$$
\begin{aligned}
& u=\varepsilon^{-1}\left[e^{\varepsilon x} \cos \varepsilon y-1\right], \\
& v=\varepsilon^{-1} e^{\varepsilon x} \sin \varepsilon y .
\end{aligned}
$$

Нетрудно проверить, что в $u v$-плоскости траектории прямые. Более того, так как преобразование (5.3) конформное, зеркальные отражения преобразуются в зеркальные отражения. В новых координатах частица движется с переменной скоростью, но это неважно, поскольку нас интересует только отображение столкновений. Тем самым наша задача сводится к биллиарду в новой области $T(\widetilde{\mathscr{D}})=\mathscr{Q} \subset \mathbb{R}^{2}$.

Периодические выпуклые рассеиватели на $x y$-плоскости отображаются в рассеиватели на $u v$-плоскости, которые не расположены периодически и размеры которых растут с ростом $x$ (благодаря множителю $e^{\varepsilon x}$ в (5.4)). Если $\kappa-$ кривизна границы рассеивателя на $x y$-плоскости, то непосредственное вычисление показывает, что кривизна ее образа на $u v$-плоскости равна $e^{-\varepsilon x}(\kappa+\mathscr{O}(\varepsilon))$, т. е. она не меняет знак при достаточно малых $\varepsilon$; отсюда следует, что новые рассеиватели остаются выпуклыми.

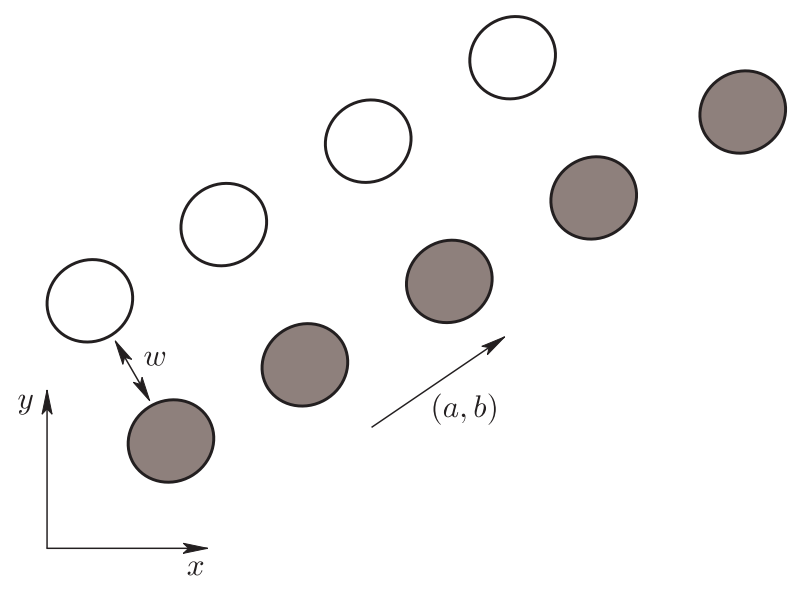

Рис. 2. Ряд рассеивателей, образующий границу бесконечного коридора

Теперь посмотрим, как бесконечный коридор на $x y$-плоскости преобразуется на $u v$-плоскости. Рассмотрим последовательность одинаковых рассеивателей, 
образующую "границу" коридора; см. серые круги на рис. 2. Каждый рассеиватель получается сдвигом предыдущего на постоянный вектор $(a, b)$, параллельный коридору; мы предполагаем для простоты, что $a \geqslant 0$ и $b>0$, как на рисунке. Рассмотрим последовательность точек $\left(x_{m}, y_{m}\right), m=1,2, \ldots$, связанных соотношениями

$$
x_{m+1}=x_{m}+a, \quad y_{m+1}=y_{m}+b .
$$

Их образы $\left(u_{m}, v_{m}\right)=T\left(x_{m}, y_{m}\right)$ удовлетворяют следующим соотношениям:

$$
\begin{aligned}
& u_{m+1}=e^{\varepsilon a}\left(u_{m} \cos \varepsilon b-v_{m} \sin \varepsilon b\right)+\varepsilon^{-1}\left(e^{\varepsilon a} \cos \varepsilon b-1\right), \\
& v_{m+1}=e^{\varepsilon a}\left(u_{m} \sin \varepsilon b+v_{m} \cos \varepsilon b\right)+\varepsilon^{-1} e^{\varepsilon a} \sin \varepsilon b .
\end{aligned}
$$

Это показывает, что соответствующие рассеиватели на $u v$-плоскости (см. рис. 3)

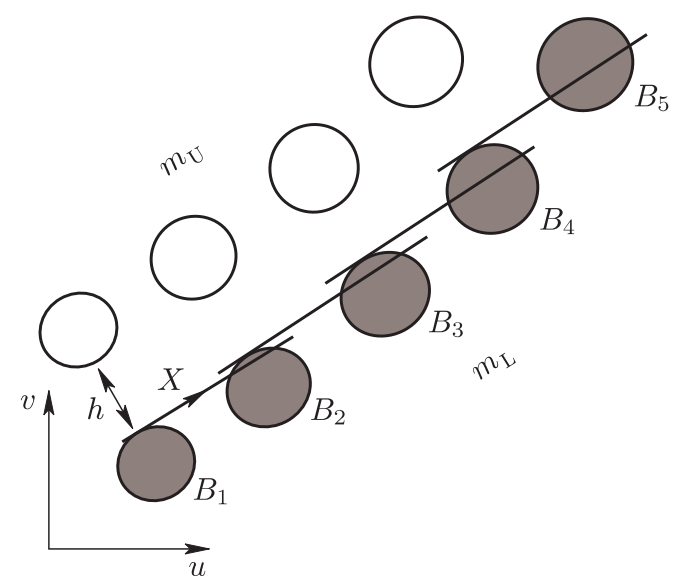

Рис. 3. Преобразованные рассеиватели на $u v$-плоскости блокируют коридор

можно рекурсивно описать следующим образом. Каждый рассеиватель $B_{n+1}$ получается из предыдущего $B_{n}$ за три шага:

1) $B_{n}$ поворачивается против часовой стрелки вокруг начала координат на угол $\varepsilon b$;

2) затем он растягивается гомотетически относительно начала координат с коэффициентом $e^{\varepsilon a}$;

3) наконец, он сдвигается на постоянный вектор

$$
\left(a^{\prime}, b^{\prime}\right)=\left(\varepsilon^{-1}\left(e^{\varepsilon a} \cos \varepsilon b-1\right), \varepsilon^{-1} e^{\varepsilon a} \sin \varepsilon b\right) .
$$

Эта последовательность операций строит следующий рассеиватель $B_{n+1}$ из $B_{n}$.

Из-за поворотов ряд рассеивателей на $u v$-плоскости "заворачивается", так что коридор между ними уже не прямой. Только конечное число рассеивателей "видны” из окрестности начала координат; отсюда следует, что биллиард в новой области $\mathscr{Q}$ имеет конечный горизонт. Рассеиватель с номером $m$ находится на расстоянии $\sim c m$ от начала координат, где $c=\sqrt{a^{2}+b^{2}}$; отсюда следует, что его поворот на угол $\varepsilon b$ будет далее блокировать обзор вдоль 
коридора на расстояние $\sim c m \varepsilon b$ в направлении, перпендикулярном к коридору. Суммарная блокировка, вызываемая $m$ рассеивателями, теперь примерно равна

$$
c b \varepsilon(1+2+\cdots+m) \sim \frac{1}{2} c b \varepsilon m^{2} .
$$

Если $w$ - ширина коридора, то максимальное число рассеивателей, видимых из начальной области в данном коридоре, равно $m_{\max } \sim \sqrt{2 w /(c b \varepsilon)}$, т. е.

$$
m_{\max } \sim C / \sqrt{\varepsilon} .
$$

Через $C$ мы будем обозначать различные абсолютные константы (не зависящие от $\varepsilon$ ), чьи значения несущественны. Заметим, что ростом размера рассеивателей можно пренебречь - последний видимый рассеиватель всего лишь в

$$
e^{\varepsilon a m_{\max }} \sim e^{\mathscr{O}(\sqrt{\varepsilon})} \sim 1+\mathscr{O}(\sqrt{\varepsilon})
$$

раз больше, чем ближайший.

Поскольку биллиард в $\mathscr{Q}$ является рассеивающим и имеет конечный горизонт, он обладает всеми стандартными свойствами [7], в частности, свойствами равномерного растяжения и сжатия, и имеет конечное число кривых разрыва. Заметим, что из растяжения в $\mathscr{Q}$ не следует автоматически растяжение в $\widetilde{\mathscr{D}}$, так как отображение $T$ изменяет масштаб расстояний (с коэффициентом $e^{\varepsilon x}$ ), но максимальный коэффициент изменения масштаба за время одного пробега между столкновениями является $e^{\mathscr{O}(\sqrt{\varepsilon})} \approx 1$, откуда следует, что у нас все еще равномерное растяжение для отображения столкновений в исходной области $\widetilde{\mathscr{D}}$.

Далее рассмотрим кривые разрыва отображения столкновений. Траектории, покидающие начальный участок бесконечного коридора и идущие в него, могут попасть на $\sim m_{\max }$ различных рассеивателей на обеих сторонах коридора. Точнее, обозначим через $m_{\mathrm{U}}$ число видимых рассеивателей на верхней стороне коридора и через $m_{\mathrm{L}}-$ на нижней стороне; см. рис. 3. Аккуратные оценки показывают, что

$$
m_{\mathrm{U}} \sim \frac{C_{1}}{\sqrt{\varepsilon}}, \quad m_{\mathrm{L}} \sim \frac{C_{2}}{\sqrt{\varepsilon}}, \quad 0<C_{1}<C_{2}
$$

тем самым и $m_{\mathrm{U}}$, и $m_{\mathrm{L}}$ порядка $m_{\max }$. Таким образом, имеется $m_{\mathrm{U}}+m_{\mathrm{L}} \sim m_{\max }$ кривых разрыва. Их число не равномерно, оно растет при $\varepsilon \rightarrow 0$, так что для них требуется тщательный анализ.

Поскольку траектории в $\mathscr{Q}$ суть прямые линии, наш анализ проводится по той же схеме, что для биллиардов с бесконечным горизонтом [7; §4.10]; он стандартен и элементарен, и мы приводим только окончательные результаты. Линии разрыва вблизи точек, помеченных $X$ на рис. 3 , показаны на рис. 4. Длинная кривая разрыва $S$ соответствует скользящим столкновениям с непосредственно следующим рассеивателем в коридоре (помеченным $B_{2}$ на рис. 3 ). Короткие кривые разрыва образованы скользящими столкновениями с другими рассеивателями в коридоре. Участки между кривыми разрыва обычно называются ячейками, они образованы траекториями, попадающими на данный рассеиватель. Таким образом, ячейки можно естественным образом занумеровать числами $1, \ldots, m_{U}$ (соответствующими верхним рассеивателям) 
и $1, \ldots, m_{\mathrm{L}}$ (для нижних рассеивателей). Соответственно обозначим ячейки через $D_{1}^{(\mathrm{U})}, \ldots, D_{m_{\mathrm{U}}}^{(\mathrm{U})}$ и $D_{1}^{(\mathrm{L})}, \ldots, D_{m_{\mathrm{L}}}^{(\mathrm{L})}$ (здесь $\mathrm{U}$ и $\mathrm{L}$ означают соответственно "Upper" и "Lower").
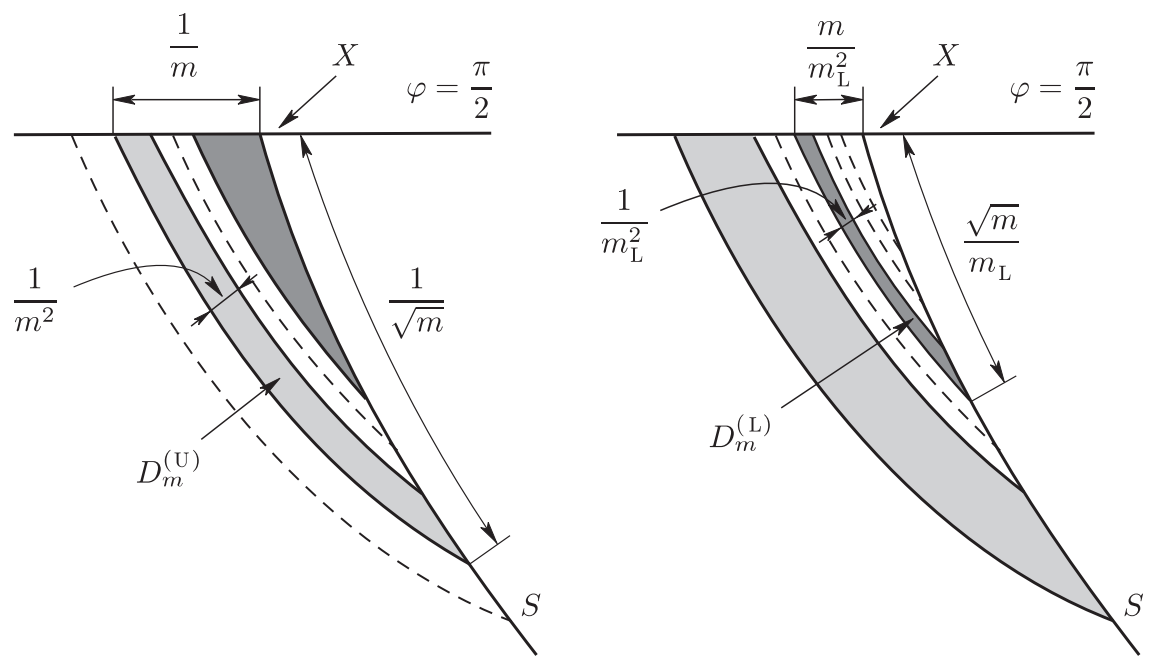

Рис. 4. Кривые разрыва и ячейки. Слева светло-серым закрашена "верхняя" ячейка $D_{m}^{(\mathrm{U})}$, показанная со всеми ее размерностями; объединение всех "нижних" ячеек $D_{m}^{(\mathrm{L})}$ закрашено темно-серым. Справа темно-серым закрашена "нижняя" ячейка $D_{m}^{(\mathrm{L})}$, показанная со всеми ее размерностями; объединение всех "верхних" ячеек $D_{m}^{(\mathrm{U})}$ закрашено светло-серым.

На рис. 4 ячейки изображены следующим образом. Первыми (дальше от $S$ ) идут ячейки $D_{1}^{(\mathrm{U})}, \ldots, D_{m_{\mathrm{U}}}^{(\mathrm{U})}$ (в этом порядке). Высота ячейки $D_{m}^{(\mathrm{U})}$ является $\mathscr{O}(1 / \sqrt{m})$, а ее ширина является $\mathscr{O}\left(1 / m^{2}\right)$, так же как в классических биллиардах с бесконечным горизонтом; см., например, [7; $\$ 4.10]$. Здесь и до конца этого раздела запись $R=\mathscr{O}\left(m^{a}\right)$ означает, что $c_{1} m^{a}<R<c_{2} m^{a}$ для некоторых констант $0<c_{1}<c_{2}<\infty$. Неустойчивые кривые внутри $D_{m}^{(\mathrm{U})}$ растягиваются с коэффициентом $\Lambda_{m}^{(\mathrm{U})}=\mathscr{O}\left(\mathrm{m}^{3 / 2}\right)$.

Вторыми (ближе к $S$ ) идут ячейки $D_{m_{\mathrm{L}}}^{(\mathrm{L})}, \ldots, D_{1}^{(\mathrm{L})}$ (в обратном порядке; ячейка $D_{1}^{(\mathrm{L})}$ примыкает к точке $\left.X\right)$. Высота ячейки $D_{m}^{(\mathrm{L})}$ является $\mathscr{O}\left(\sqrt{m} / m_{\mathrm{L}}\right)$, а ее ширина является $\mathscr{O}\left(1 / m_{\mathrm{L}}^{2}\right)$. Неустойчивые кривые внутри $D_{m}^{(\mathrm{L})}$ растягиваются с коэффициентом $\Lambda_{m}^{(\mathrm{L})}=\mathscr{O}\left(m_{\mathrm{L}} \sqrt{m}\right)$. Заметим, что отображение столкновений $\mathscr{F}_{\varepsilon}$ преобразует каждую ячейку $D$ в похожую ячейку, см. рис. 5 , с той разницей, что оно растягивает более короткие стороны $D$ и сжимает ее более длинные стороны, так что коэффициент растяжения можно аппроксимировать как

$$
\Lambda \sim \frac{\text { высота } D}{\text { ширина } D} ;
$$

это соотношение является стандартным при изучении рассеивающих биллиардов [7]. 

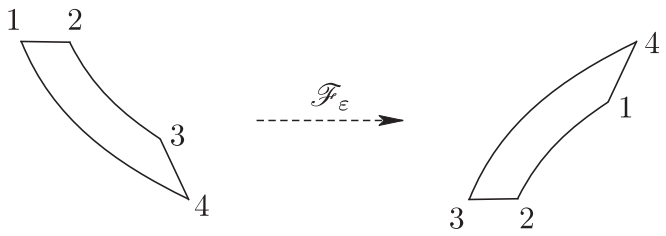

Рис. 5. Отображение $\mathscr{F}_{\varepsilon}$ преобразует ячейку $D_{m}^{(\mathrm{U})}$ в похожую область. Углы $D_{m}^{(\mathrm{U})}$ и их образы занумерованы, чтобы обозначить действие $\mathscr{F}_{\varepsilon}$.

Запишем следующие формулы для мер ячеек:

$$
\nu_{0}\left(D_{m}^{(\mathrm{U})}\right)=\mathscr{O}\left(\frac{1}{m^{3}}\right), \quad \nu_{0}\left(D_{m}^{(\mathrm{L})}\right)=\mathscr{O}\left(\frac{m}{m_{\mathrm{L}}^{4}}\right) .
$$

Действительно, биллиардная мера $\nu_{0}$ является гладкой с плотностью $\cos \varphi$, поэтому мера каждой ячейки имеет порядок величины произведения ширина $\times(\text { высота })^{2}$.

\section{6. Леммы о росте}

Наше отображение $\mathscr{F}_{\varepsilon}: \mathscr{M} \rightarrow \mathscr{M}$ является малым возмущением рассеивающего биллиардного отображения. Последнее обладает свойством равномерно сильного растяжения, которое уравновешивается некоторыми слабыми сингулярностями. Изучение таких отображений хорошо развито, см. [1], [21], [12], [13], [17], [15], [25], но пока нет аксиоматической теории, которая покрывала бы все такие модели - каждый новый случай выглядит немного отличным от других - поэтому в какой-то момент нам нужно будет приспособить общие рассуждения к нашему частному случаю. Мы дадим только схему общих шагов этой процедуры, которые не нуждаются в адаптации, отсылая к исходным статьям за деталями, и сосредоточимся на новых особенностях для нашей модели.

Неустойчивые и устойчивые конусы и соответственно неустойчивые и устойчивые кривые для отображения столкновений в рассеивающих биллиардах определяются стандартным образом [7; гл. 4]. Итак, у нас есть эти объекты для биллиарда в $\mathscr{Q}$, а преобразование их обратно в $\mathscr{D}$ под действием $T^{-1}$ дает все такие объекты для нашего отображения $\mathscr{F}$. По-другому неустойчивые кривые для $\mathscr{F}_{\varepsilon}$ можно построить напрямую, например, используя "сильно расходящиеся семейства линий потока", определяемые в [12; с. 213].

Далее, можно предполагать, как в [12; с. 215], что кривизна неустойчивых и устойчивых кривых равномерно ограничена. Чтобы обеспечить контроль за искажением, построим стандартным образом полосы однородности:

$$
\begin{aligned}
\mathbb{H}_{j} & =\left\{(r, \varphi): \pi / 2-j^{-2}<\varphi<\pi / 2-(j+1)^{-2}\right\} \quad \forall j \geqslant j_{0}, \\
\mathbb{H}_{0} & =\left\{(r, \varphi):-\pi / 2+j_{0}^{-2}<\varphi<\pi / 2-j_{0}^{-2}\right\}, \\
\mathbb{H}_{-j} & =\left\{(r, \varphi):-\pi / 2+(j+1)^{-2}<\varphi<-\pi / 2+j^{-2}\right\} \quad \forall j \geqslant j_{0},
\end{aligned}
$$

где $j_{0}>1$ - большая константа, см. [12; с. 216] и [7; § 5.3]. Разрежем $\mathscr{M}$ вдоль их границ, так что все наши устойчивые и неустойчивые кривые автоматически 
станут однородными. Образы и прообразы этих границ становятся линиями разрыва (сингулярностями) соответственно для $\mathscr{F}_{\varepsilon}^{-1}$ и $\mathscr{F}_{\varepsilon}$. Для любой неустойчивой кривой $W$ и любой точки $X \in W$ обозначим через $\mathscr{J}_{W} \mathscr{F}_{\varepsilon}^{n}(X)$ якобиан отображения $\mathscr{F}_{\varepsilon}^{n}$, ограниченного на $W$, в точке $X$. Если $\mathscr{F}_{\varepsilon}^{i}(W)$ - неустойчивая кривая для всех $0 \leqslant i \leqslant n$, то имеем следующую оценку искажсеня (см. $[12$; лемма 4.2]):

$$
\left|\log \mathscr{J}_{W} \mathscr{F}_{\varepsilon}^{n}(X)-\log \mathscr{J}_{W} \mathscr{F}_{\varepsilon}^{n}(Y)\right| \leqslant C\left|\mathscr{F}_{\varepsilon}^{n}(W)\right|^{1 / 3}, \quad X, Y \in W,
$$

где $|W|$ обозначает длину $W$. Соответственно, если $W^{\mathrm{u}}-$ однородное неустойчивое многообразие (называемое "h-fiber" в [12]) и $\rho_{W^{u}}-\mathrm{u}-\mathrm{SRB}$-плотность на $W^{\mathrm{u}}$, т. е. единственная вероятностная плотность, удовлетворяющая

$$
\frac{\rho_{W^{\mathrm{u}}}(X)}{\rho_{W^{\mathrm{u}}}(Y)}=\lim _{n \rightarrow \infty} \frac{\mathscr{J}_{W^{\mathrm{u}}} \mathscr{F}_{\varepsilon}^{-n}(X)}{\mathscr{J}_{W^{\mathrm{u}}} \mathscr{F}_{\varepsilon}^{-n}(Y)}, \quad X, Y \in W^{\mathrm{u}},
$$

то из (6.2) следует, что $\left|\frac{d}{d X} \log \rho_{W^{\text {u }}}(X)\right| \leqslant C\left|W^{\mathrm{u}}\right|^{-2 / 3}$ (см. [7; § 5.6] и [13]).

Если $W_{1}, W_{2}$ - неустойчивые кривые, а $\xi$ - устойчивое многообразие, пересекающее каждую $W_{i}$ по точке $X_{i}$, то якобиан отображения голономии $\mathbf{h}$ : $W_{1} \rightarrow W_{2}$ в точке $X_{1}$ удовлетворяет

$$
e^{-C\left(\beta+\delta^{1 / 3}\right)} \leqslant \mathscr{J} \mathbf{h}\left(X_{1}\right) \leqslant e^{C\left(\beta+\delta^{1 / 3}\right)},
$$

где $\delta=\left|\xi\left(X_{1}, X_{2}\right)\right|$ - длина отрезка из $\xi$ между $X_{1}$ и $X_{2}$, а $\beta$ - угол между касательными векторами к $W_{1}$ и $W_{2}$ соответственно в точках $X_{1}$ и $X_{2}$ (см. [12; лемма 4.3], [7; теорема 5.42] или [13]).

Множество сингулярности $\mathscr{S} \subset \mathscr{M}$ отображения $\mathscr{F}_{\varepsilon}$ является счетным объединением устойчивых кривых с равномерно ограниченной кривизной. Они обладают обычными свойствами “продолжения" и “выравнивания" (см. [7; гл. 4]), т. е. они разбивают $\mathscr{M}$ на криволинейные многоугольники с внутренними углами $<\pi$.

Для данных $X, Y \in \mathscr{M}$ обозначим через $\mathbf{s}_{+}(X, Y) \geqslant 0$ время будущего разделения (первый момент времени, когда образы $\mathscr{F}_{\varepsilon}^{n}(X)$ и $\mathscr{F}_{\varepsilon}^{n}(Y)$ попадут на различные рассеиватели или в различные полосы однородности, т. е. первый момент, когда $\mathscr{F}_{\varepsilon}^{n-1}(X)$ и $\mathscr{F}_{\varepsilon}^{n-1}(Y)$ будут принадлежать различным компонентам связности из $\mathscr{M} \backslash \mathscr{S})$, и аналогично пусть $\mathbf{s}_{-}(X, Y) \geqslant 0$ обозначает время прошлого разделения. Заметим, что если $X$ и $Y$ лежат на одной неустойчивой кривой $W \subset \mathscr{M}$, то $|W(X, Y)| \leqslant C \Lambda^{-\mathbf{s}_{+}(X, Y)}$, где $\Lambda>1-$ константа гиперболичности для $\mathscr{F}_{\varepsilon}$ (cp. [7; (5.32)]). Теперь из (6.4) следует (см., например, [7; предложение 5.48]), что для любых $X, Y \in W_{1}$

$$
|\log \mathscr{J} \mathbf{h}(X)-\log \mathscr{J} \mathbf{h}(Y)| \leqslant C \vartheta^{\mathbf{s}_{+}(X, Y)},
$$

где $\vartheta=\Lambda^{-1 / 6}<1$. Следуя Янг [9; с. 597], назовем свойство (6.5) “динамически определенной гёльдеровской непрерывностью" функции $\mathscr{J} \mathbf{h}$.

Далее мы выведем ключевой факт о росте неустойчивых кривых, известный как лемма о росте (Growth Lemma). Для данной неустойчивой кривой $W$ обозначим через $W_{i} \subset W$ компоненты связности из $W \backslash \mathscr{S}$, т. е. отрезки из $W$, на которых $\mathscr{F}_{\varepsilon}$ гладкое, а через $\Lambda_{i}-$ (минимальный) коэффициент растяжения $W_{i}$ 
под действием $\mathscr{F}_{\varepsilon}$. Тогда найдется метрика в $\mathscr{M}$, равномерно эквивалентная евклидовой метрике $d l^{2}=d r^{2}+d \varphi^{2}$ и такая, что

$$
\liminf _{\delta_{0} \rightarrow 0} \sup _{W:|W|<\delta_{0}} \sum_{i} \Lambda_{i}^{-1}<1
$$

где супремум берется по неустойчивым кривым $W$ длины $<\delta_{0}$. Оценка $(6.6)$ называется оценкой одношагового растяжения [25; §5], она показывает, насколько неустойчивые кривые растягиваются под действием одной итерации отображения.

Для рассеивающих биллиардов с конечным горизонтом доказательство оценки (6.6) стандартно (см. [7; лемма 5.56]), и оно легко переносится на возмущенные биллиарды (см. [17; лемма 4.10]), такие, как системы с малыми внешними силами. В нашем случае бесконечные коридоры добавляют новые осложнения - может случиться, что короткая неустойчивая кривая $W$ пересечет много ячеек, описанных в предыдущем разделе (так бывает, когда образы кусочков $W_{i}$ движутся в бесконечный коридор и попадают на разные и удаленные рассеиватели). Предположим, что $W$ пересекает ячейки $D_{p}^{(\mathrm{U})}, p_{0} \leqslant p \leqslant m_{\mathrm{U}}$, и $D_{q}^{(\mathrm{L})}, q_{0} \leqslant q \leqslant m_{\mathrm{L}}$ (индексы $\mathrm{U}$ и L были пояснены в предыдущем разделе); тогда

$$
\sum_{i} \Lambda_{i}^{-1} \leqslant \sum_{p=p_{0}}^{m_{\mathrm{U}}} \frac{C}{p^{3 / 2}}+\sum_{q=q_{0}}^{m_{\mathrm{L}}} \frac{C}{m_{\mathrm{L}} q^{1 / 2}} \leqslant \frac{C}{p_{0}^{1 / 2}}+\frac{C}{m_{\mathrm{L}}^{1 / 2}} .
$$

Если $W$ мала, то $p_{0}$ должно быть большим, и приведенная выше оценка $\ll 1$. Это завершает доказательство оценки (6.6).

Из оценки одношагового растяжения (6.6) вытекает ряд свойств, в совокупности известных как леммы о росте (Growth Lemmas); см. [7; глава 5], [7; § 4.7], [12; предложение 5.3], [16; лемма 4.10] и доказательства в этих работах. Сформулируем ниже их наиболее общеупотребительные варианты.

Для данной неустойчивой кривой $W$ обозначим через $m_{W}$ меру Лебега на ней. Для любого $n \geqslant 0$ образ $\mathscr{F}_{\varepsilon}^{n}(W)$ этой кривой является конечным или счетным объединением однородных неустойчивых кривых, называемых h-компонентами, и для любого $X \in W$ обозначим через $W_{n}(X)$ h-компоненту, содержащую точку $\mathscr{F}_{\varepsilon}^{n}(X)$. Теперь обозначим через

$$
r_{n}(X)=r_{W_{n}(X)}\left(\mathscr{F}_{\varepsilon}^{n}(X)\right)
$$

расстояние от $\mathscr{F}_{\varepsilon}^{n}(X)$ до ближайшего конца $W_{n}(X)$. Ясно, что $r_{n}$ является функцией на $W$, характеризующей размер h-компонент из $\mathscr{F}_{\varepsilon}^{n}(W)$. Также обозначим через $\Lambda>1$ константу гиперболичности, т. е. минимальный коэффициент растяжения неустойчивых кривых.

ЛЕмма 6.1 (лемма о росте). (а) Существуют такие константы $\vartheta_{0} \in(0,1)$ $u c_{1}, c_{2}>0$, что для всех $n \geqslant 0 u \epsilon>0$

$$
m_{W}\left(r_{n}(X)<\epsilon\right) \leqslant c_{1}\left(\vartheta_{0} \Lambda\right)^{n} m_{W}\left(r_{0}<\epsilon / \Lambda^{n}\right)+c_{2} \epsilon m_{W}(W) .
$$

(б) Существуют такие константы $c_{3}, c_{4}>0$, что если $n \geqslant c_{3}\left|\log m_{W}(W)\right|$, то для любого $\epsilon>0$ имеем $m_{W}\left(r_{n}(X)<\epsilon\right) \leqslant c_{4} \epsilon m_{W}(W)$. 
(в) Существуют такие константы $\vartheta_{1} \in(0,1), c_{5}, c_{6}>0$ и малое $\epsilon_{0}>0$, что для любых $n_{2}>n_{1}>c_{5}\left|\log m_{W}(W)\right|$ имеем

$$
m_{W}\left(\max _{n_{1}<n<n_{2}} r_{n}(X)<\epsilon_{0}\right) \leqslant c_{6} \vartheta_{1}^{n_{2}-n_{1}} m_{W}(W) .
$$

За доказательством и следствиями этой леммы мы отсылаем читателя к [7], [25]. Подчеркнем, что $\liminf$ в (6.6) и все константы в лемме о росте не зависят от $\varepsilon$, т. е. соответствующие свойства выполняются равномерно по нашему параметру модели $\varepsilon$.

Далее определим класс вероятностных мер с носителем на неустойчивых кривых. Стандартная пара $\ell=(W, \rho)$ - это неустойчивая кривая $W \subset \mathscr{M}$ с вероятностной мерой $\mathrm{P}_{\ell}$ на ней, плотность $\rho$ которой (относительно меры Лебега на $W$ ) удовлетворяет соотношению

$$
|\log \rho(X)-\log \rho(Y)| \leqslant C_{\mathrm{r}} \Lambda^{-\mathbf{s}_{+}(X, Y)} .
$$

Здесь $C_{\mathrm{r}}>0$ - достаточно большая константа (не зависящая от $\varepsilon$ ). Для любой стандартной пары $\ell=(W, \rho)$ и для любого $n \geqslant 1$ образ $\mathscr{F}_{\varepsilon}^{n}(W)$ является конечным или счетным объединением h-компонент, на которых плотность меры $\mathscr{F}_{\varepsilon}^{n}\left(\mathrm{P}_{\ell}\right)$ удовлетворяет (6.8); отсюда следует, что образ стандартной пары под действием $\mathscr{F}_{\varepsilon}^{n}-$ семейство стандартных пар (с фактормерой).

Более общо, стандартное семейство - это произвольное (счетное или несчетное) множество $\mathscr{G}=\left\{\ell_{\alpha}\right\}=\left\{\left(W_{\alpha}, \rho_{\alpha}\right)\right\}, \alpha \in \mathfrak{A}$, стандартных пар с вероятностной фактормерой $\lambda_{\mathfrak{g}}$ на множестве индексов $\mathfrak{A}$. Такое семейство индуцирует вероятностную меру $\mathrm{P}_{\mathscr{G}}$ на объединении $\bigcup_{\alpha} W_{\alpha}$ (и тем самым на $\mathscr{M}$ ), определяемую следующим образом:

$$
\mathrm{P}_{\mathscr{G}}(B)=\int \mathrm{P}_{\alpha}\left(B \cap W_{\alpha}\right) d \lambda_{\mathscr{G}}(\alpha) \quad \forall B \subset \mathscr{M} .
$$

Любое стандартное семейство $\mathscr{G}$ отображается под действием $\mathscr{F}_{\varepsilon}^{n}$ в другое стандартное семейство $\mathscr{G}_{n}=\mathscr{F}_{\varepsilon}^{n}(\mathscr{G})$, причем $\mathrm{P}_{\mathscr{G}_{n}}=\mathscr{F}_{\varepsilon}^{n}(\mathrm{P} \mathscr{G})$.

Для каждого $\alpha \in \mathfrak{A}$ любая точка $X \in W_{\alpha}$ делит кривую $W_{\alpha}$ на два куска, и мы обозначаем через $r_{\mathscr{G}}(X)$ длину более короткого из них. Теперь величина $\mathscr{Z}_{\mathscr{G}}=\sup _{\epsilon>0} \epsilon^{-1} \mathrm{P}_{\mathscr{G}}\left(r_{\mathscr{G}}<\epsilon\right)$ отражает “средний” размер кривых $W_{\alpha}$ в $\mathscr{G}$, и мы имеем

$$
\mathscr{Z}_{\mathscr{G}} \leqslant C \int \frac{d \lambda_{\mathscr{G}}(\alpha)}{\left|W_{\alpha}\right|} ;
$$

см. [7; упражнение 7.15]. Мы рассматриваем только стандартные семейства, для которых $\mathscr{Z}_{\mathscr{G}}<\infty$. Из леммы о росте следует, что для всех $n \geqslant 0$ и для некоторой константы $\theta \in(0,1)$

$$
\mathscr{Z}_{\mathscr{G}_{n}} \leqslant C\left(\theta^{n} \mathscr{Z}_{\mathscr{G}}+1\right)
$$

(доказательство см. в [7; предложение 7.17]); эта оценка по существу утверждает, что стандартные семейства растут под действием $\mathscr{F}_{\varepsilon}^{n}$ экспоненциально быстро.

Стандартная пара $(W, \rho)$ называется собственной, если $|W| \geqslant \delta_{\mathrm{p}}$, где $\delta_{\mathrm{p}}>0-$ малая, но фиксированная константа. Стандартное семейство $\mathscr{G}$ называется 
собственным, если $\mathscr{Z}_{\mathscr{g}} \leqslant C_{\mathrm{p}}$, где $C_{\mathrm{p}}$ - большая, но фиксированная константа (выбранная так, чтобы семейство, состоящее из одной собственной стандартной пары, было собственным как семейство). Образ собственного стандартного семейства под действием $\mathscr{F}_{\varepsilon}^{n}$ является собственным для любого $n \geqslant 1$.

Гладкое слоение $\mathscr{M}$ (достаточно длинными) неустойчивыми кривыми дает собственное стандартное семейство $\mathscr{G}_{0}$, для которого $\mathrm{P}_{\mathscr{G}_{0}}=\nu_{0}$ есть биллиардная инвариантная мера (см. [7; с. 172]). Кроме того, имеется особое стандартное семейство $\mathscr{E}$, состоящее из (максимальных) неустойчивых многообразий $W^{\mathrm{u}}$ для отображения $\mathscr{F}_{\varepsilon}$ с SRB-плотностями $\rho_{W}$ и на них и с фактормерой, порожденной мерой $\nu_{\varepsilon}$; в этом случае $\mathrm{P}_{\mathscr{E}}=\nu_{\varepsilon}$, а семейство $\mathscr{E}$ является собственным (в силу [12; предложение 5.6]) и, очевидно, $\mathscr{F}_{\varepsilon}$-инвариантным.

\section{7. Эргодические и статистические свойства}

Нашей следующей задачей является доказательство существования и единственности меры Синая-Рюэля-Боуэна (SRB-меры) $\nu_{\varepsilon}$ на $\mathscr{M}$, которая по определению является $\mathscr{F}$-инвариантной эргодической мерой, условные распределения которой на неустойчивых многообразиях абсолютно непрерывны (и тогда их условные плотности даются формулой (6.3)).

Лемма о росте может быть использована для оценки меры Лебега $\epsilon$-окрестности множества сингулярности и его прообразов (см. детали в [12; с. 228]), и тогда из стандартных результатов, например, [26], [27], следует существование и конечность SRB-мер. Конечность здесь означает, что имеется только конечное число SRB-мер, и каждая из них обладает свойствами перемешивания и Бернулли с точностью до конечного цикла. Из общих результатов также следует, что для каждой SRB-меры $\nu$ имеется множество $B=B_{\nu} \subset \mathscr{M}$ положительной меры Лебега ("область притяжения") такое, что для любого $X \in B$ и любой непрерывной функции $f: \mathscr{M} \rightarrow \mathbb{R}$ имеет место сходимость

$$
\frac{1}{n}\left[f(X)+f(T X)+\cdots+f\left(T^{n-1} X\right)\right] \rightarrow \int_{\mathscr{M}} f(X) d \nu
$$

при $n \rightarrow \infty$, т. е. SRB-мера $\nu$ описывает распределение случайно выбранной траектории с положительной вероятностью; по этой причине говорят, что любая SRB-мера физически наблюдаема.

Единственность и свойство перемешивания SRB-меры требуют более тонкого рассуждения; оно основано на том, что наше отображение $\mathscr{F} \varepsilon$ является малым возмущением сильно перемешивающего отображения $\mathscr{F}$ с гладкой инвариантной мерой $\nu_{0}$. Это рассуждение приведено во всех деталях в [12; c. 229-233], здесь мы только напомним его главные шаги.

Сначала отыскивается область $R \subset \mathscr{M}$, ограниченная двумя устойчивыми и двумя неустойчивыми кривыми (криволинейный “ромб”), для которой образ любой неустойчивой кривой $W \subset \mathscr{M}$ под действием биллиардного отображения $\mathscr{F}$ достаточно часто полностью пересекает $R$ (пересекая обе ее устойчивые стороны); такая область $R$ построена в [6], см. более свежее изложение этого построения в $[7 ; \S 7.12]$. Лемма о росте также гарантирует, что имеется достаточно много устойчивых и неустойчивых многообразий, полностью пересекающих область $R$, т. е. $R$ “насыщена" устойчивыми и неустойчивыми многообразиями. 
Затем используется рассуждение о возмущении (см. [1; лемма 13] и [12; c. 230]), чтобы распространить вышеупомянутое свойство на отображения $\mathscr{F} \varepsilon$ для всех достаточно малых $\varepsilon$. Частота, с которой образы кривой $W$ возвращаются к $R$, ограничена снизу константой, равномерной по $\varepsilon$. Теперь для любых двух неустойчивых многообразий $W_{1}, W_{2} \subset \mathscr{M}$ для отображения $\mathscr{F} \varepsilon$ их образы под действием $\mathscr{F}_{\varepsilon}^{n}$ для всех достаточно больших $n$ полностью пересекают $R$, откуда следует, что их можно соединить достаточным числом устойчивых многообразий отображения $\mathscr{F}_{\varepsilon}$. Отсюда легко следуют единственность и свойство перемешивания SRB-меры $\nu_{\varepsilon}$, откуда следует ее свойство Бернулли.

В качестве побочного результата получаем, что область притяжения меры $\nu_{\varepsilon}$ имеет полную меру Лебега, а в силу свойства перемешивания меры $\nu_{\varepsilon}$ выполнено равенство $\nu_{\varepsilon}=\lim _{n \rightarrow \infty} \mathscr{F}_{\varepsilon}^{n} \nu_{0}$ в смысле слабой сходимости; здесь $\nu_{0}$ обозначает $\mathscr{F}$-инвариантную меру, которая имеет гладкую плотность, но можно заменить $\nu_{0}$ любой другой мерой с гладкой положительной плотностью на $\mathscr{M}$. Вскоре мы приведем даже еще более сильный вариант этого свойства (теорема 10).

Теперь займемся статистическими свойствами системы $\left(\mathscr{M}, \mathscr{F}_{\varepsilon}, \nu_{\varepsilon}\right)$. Можно использовать устойчивые и неустойчивые многообразия, полностью пересекающие ромб $R$, в качестве базиса для башни Янг и затем доказать экспоненциальную оценку хвоста для времен возврата с помощью леммы о росте, как это сделано в [21], [25], [28]. Это дает экспоненциальное убывание корреляций.

Или же можно использовать устойчивые многообразия в $R$ для спаривания образов неустойчивых кривых и доказать так называемую лемму о спаривании, снова в качестве основного инструмента использующую лемму о росте. Этот подход применяется в [17], [7], [13], [28]. Ниже мы сформулируем наиболее важные следствия этой техники.

Будем говорить, что функция $f: \mathscr{M} \rightarrow \mathbb{R}$ динамически гёльдеровски непрерывна, если существуют такие $\vartheta_{f} \in(0,1)$ и $K_{f}>0$, что для любых $X$ и $Y$, лежащих на одной неустойчивой кривой, выполняется неравенство

$$
|f(X)-f(Y)| \leqslant K_{f} \vartheta_{f}^{\mathbf{s}_{+}(X, Y)}
$$

и для любых $X$ и $Y$, лежащих на одной устойчивой кривой, выполняется неравенство

$$
|f(X)-f(Y)| \leqslant K_{f} \vartheta_{f}^{\mathbf{s}_{-}}(X, Y) .
$$

Обозначим пространство таких функций через $\mathscr{H}$. Оно содержит любую кусочно гёльдеровски непрерывную функцию, разрывы которой совпадают с разрывами функции $\mathscr{F}_{\varepsilon}^{ \pm m}$ для некоторого $m>0$. Например, компоненты $\Delta_{\varepsilon, x}, \Delta_{\varepsilon, y}$ вектор-функции смещения $\boldsymbol{\Delta}_{\varepsilon}$ принадлежат $\mathscr{H}$ (см. следующий раздел).

Теорема 10 (равнораспределение). Пусть $\mathscr{G}$ - собственное стандартное семейство. Для любой динамически гёльдеровски непрерывной функиии $f \in \mathscr{H}$ и для любого $n \geqslant 0$ выполняется

$$
\left|\int_{\mathscr{M}} f \circ \mathscr{F}_{\varepsilon}^{n} d \mathrm{P}_{\mathscr{G}}-\int_{\mathscr{M}} f d \nu_{\varepsilon}\right| \leqslant B_{f} \theta_{f}^{n},
$$

где $B_{f}=2 C\left(K_{f}+\|f\|_{\infty}\right) u \theta_{f}=\left[\max \left\{\vartheta_{1}, \vartheta_{f}\right\}\right]^{1 / 2}<1 ;$ здесъ $C>0 u \vartheta_{1} \in(0,1)-$ константы, не зависящие от $\mathscr{G} u \varepsilon$. 
Иными словами, итерации мер на стандартных парах слабо сходятся к SRBмере $\nu_{\varepsilon}$, причем сходимость экспоненциально быстрая в смысле (7.3).

ТЕорема 11 (экспоненциальная оценка корреляций). Для любой пары $\partial u$ намически гёльдеровски непрерывных функиий $f, g \in \mathscr{H}$ и для любого $n>0$ выполняется неравенство

$$
\left|\nu_{\varepsilon}\left(f \cdot\left(g \circ \mathscr{F}_{\varepsilon}^{n}\right)\right)-\nu_{\varepsilon}(f) \nu_{\varepsilon}(g)\right| \leqslant B_{f, g} \theta_{f, g}^{n},
$$

где

$$
\theta_{f, g}=\left[\max \left\{\vartheta_{2}, \vartheta_{f}, \vartheta_{g}\right\}\right]^{1 / 4}<1
$$

(здесь $\vartheta_{2} \in(0,1)$ - константа, не зависящая от $\left.\varepsilon\right) u$

$$
B_{f, g}=C\left(K_{f}\|g\|_{\infty}+K_{g}\|f\|_{\infty}+\|f\|_{\infty}\|g\|_{\infty}\right) .
$$

ЗАмечАниЕ 7.1. Инвариантную меру $\nu_{\varepsilon}$ в (7.4) можно заменить любой мерой $\mathrm{P}_{\mathscr{G}}$ с носителем, являющимся собственным стандартным семейством (в частности, $\mathscr{F}$-инвариантной мерой $\nu_{0}$ ). В этом случае (7.4) принимает вид

$$
\left|\mathrm{P}_{\mathscr{G}}\left(f \cdot\left(g \circ \mathscr{F}_{\varepsilon}^{n}\right)\right)-\mathrm{P}_{\mathscr{G}}(f) \nu_{\varepsilon}(g)\right| \leqslant B_{f, g} \theta_{f, g}^{n} .
$$

Доказательство этого является всего лишь несложной адаптацией стандартного доказательства вышеприведенной теоремы (см., например, доказательство теоремы 3.37 в [7]).

Последнюю теорему можно распространить на множественные корреляции, и из нее с помощью стандартного рассуждения следуют центральная предельная теорема, принцип инвариантности почти наверное и закон повторного логарифма для отображения $\mathscr{F}_{\varepsilon}$ (см. [7; гл. 7] и [13]). Рассуждение, приведенное в $[13 ; \S 3]$, также дает свойство Бернулли для потока $\Phi_{\varepsilon}^{t}$, т. е. для частицы, движущейся в области $\mathscr{D}$ с непрерывным временем.

Наконец, выведем формулу типа Кавасаки (4.1) для любой динамически гёльдеровски непрерывной функции $f \in \mathscr{H}$. Напомним, что

$$
\nu_{\varepsilon}(f)=\nu_{0}(f)+\lim _{n \rightarrow \infty} \sum_{k=1}^{n} \nu_{0}\left[\left(f \circ \mathscr{F}_{\varepsilon}^{k}\right)-\left(f \circ \mathscr{F}_{\varepsilon}^{k-1}\right)\right] .
$$

Также напомним, что $\nu_{0}=\mathrm{P}_{\mathscr{G}_{0}}$ для собственного стандартного семейства $\mathscr{G}_{0}$ (см. конец раздела 6). Итак, в силу свойства равнораспределения (теорема 10) последовательность $\nu_{0}\left(f \circ \mathscr{F}_{\varepsilon}^{k}\right)$ сходится к $\nu_{\varepsilon}(f)$ экспоненциально быстро. Поэтому ряд в (4.1) сходится с экспоненциальной скоростью, причем имеет место следующая оценка хвоста:

$$
\left|\sum_{k=n}^{\infty} \nu_{0}\left[\left(f \circ \mathscr{F}_{\varepsilon}^{k}\right)(1-g)\right]\right| \leqslant \frac{2 B_{f} \theta_{f}^{n}}{\left(1-\theta_{f}\right)} .
$$

Заметим, что если гёльдеровский показатель $\log \vartheta_{f}$ функции $f$, ее гёльдеровская норма $K_{f}$ и ее $\infty$-норма $\|f\|_{\infty}$ не зависят от $\varepsilon$, то вышеприведенная оценка также равномерна по $\varepsilon$. 


\section{8. Свойства функции $\Delta_{\varepsilon}$}

Результаты в предыдущих двух разделах были стандартны, и потому мы опустили доказательства. Начиная с этого момента наши рассуждения будут весьма специфичны для рассматриваемой модели, поэтому мы даем подробные доказательства.

Во-первых, оценим меры наших ячеек $D_{m}^{(\mathrm{U})}$ и $D_{m}^{(\mathrm{L})}$, введенных в разделе 5 , обобщая (5.7).

ЛЕмма 8.1. Для каждого $n \geqslant 0$ имеем

$$
\left(\mathscr{F}_{\varepsilon}^{n} \nu_{0}\right)\left(D_{m}^{(\mathrm{U})}\right) \sim \frac{C}{m^{3}}, \quad\left(\mathscr{F}_{\varepsilon}^{n} \nu_{0}\right)\left(D_{m}^{(\mathrm{L})}\right) \sim \frac{C m}{m_{\mathrm{L}}^{4}},
$$

и такие же оценки справедливы для пределъной меры $\nu_{\varepsilon}$.

ДокАЗАТЕЛЬСтво. При $n=0$ эти оценки были получены в (5.7). Напомним, что якобиан преобразования $\mathscr{F}_{\varepsilon}$ равен $\exp \left(-\varepsilon \Delta_{\varepsilon, x}\right)$, ср. с $(4.2) ;$ отсюда следует, что якобиан преобразования $\mathscr{F}_{\varepsilon}^{n}$ равен

$$
\frac{d \mathscr{F}_{\varepsilon}^{-n}}{d \nu_{0}}=\exp \left(-\varepsilon \sum_{k=0}^{n-1} \Delta_{\varepsilon, x} \circ \mathscr{F}_{\varepsilon}^{k}\right)
$$

Поскольку $\left\|\Delta_{\varepsilon, x}\right\|_{\infty} \leqslant C / \sqrt{\varepsilon}$ в силу (5.6), якобиан преобразования $\mathscr{F}_{\varepsilon}^{n}$ будет находиться, скажем, в интервале $[0.5,2]$ для всех $n \leqslant c / \sqrt{\varepsilon}$, если $c>0$ - достаточно малая константа. Это доказывает (8.1) для всех $n \leqslant c / \sqrt{\varepsilon}$.

Для больших значений $n$ воспользуемся свойством равнораспределения (теорема 10); применим его к функции $f=\mathbf{1}_{D}$, где $\mathbf{1}_{D}$ обозначает индикаторную функцию ячейки $D=D_{m}^{(\mathrm{U})}$ или $D=D_{m}^{(\mathrm{L})}$. Ясно, что $f$ динамически гёльдеровски непрерывна с любым $\vartheta_{f}<1$ и с $K_{f}=1$; поэтому в силу (7.3) выполняется неравенство

$$
\left|\left(\mathscr{F}_{\varepsilon}^{n} \nu_{0}\right)(D)-\nu_{\varepsilon}(D)\right| \leqslant C \theta^{n}
$$

для некоторых абсолютных констант $C>0$ и $\theta \in(0,1)$. Теперь при $n=c / \sqrt{\varepsilon}$ правая часть равна $\theta^{c / \sqrt{\varepsilon}}$, и для всех малых $\varepsilon$ имеем

$$
\theta^{c / \sqrt{\varepsilon}} \ll \varepsilon^{3 / 2} \leqslant \frac{C}{m_{\mathrm{U}}^{3}} \leqslant \min \left\{\frac{C}{m^{3}}, \frac{C m}{m_{\mathrm{L}}^{4}}\right\},
$$

что завершает доказательство леммы.

Теперь проверим, что компоненты $\Delta_{\varepsilon, x}$ и $\Delta_{\varepsilon, y}$ вектор-функции смещения $\boldsymbol{\Delta}_{\varepsilon}$ динамически гёльдеровски непрерывны, т. е. лежат в $\mathscr{H}$.

Лемма 8.2. Пусть $X, Y \in \mathscr{M}$ - две близкие точки такие, что их траектории в $\widetilde{D}$ при следующем столкновении попадают на один и тот же рассеиватель в $\mathbb{R}^{2}$. Тогда имеем

$$
\left\|\boldsymbol{\Delta}_{\varepsilon}(X)-\boldsymbol{\Delta}_{\varepsilon}(Y)\right\| \leqslant C \sqrt{\left\|\boldsymbol{\Delta}_{\varepsilon}(X)\right\| \cdot \operatorname{dist}(X, Y)},
$$

әде $C>0$ - константа, не зависящая от $\varepsilon$. 
ДокАЗАТЕЛЬСтво. Предположим на минуту, что мы имеем дело с рассеивающим биллиардом с гладкой границей в $\mathbb{R}^{2}$, т. е. с частицей, движущейся между бесконечным числом гладких выпуклых рассеивателей, кривизна $\varkappa$ которых ограничена двумя константами:

$$
0<\varkappa_{\min } \leqslant \varkappa \leqslant \varkappa_{\max }<\infty .
$$

Пусть $X=\left(\mathbf{q}_{1}, \mathbf{v}_{1}\right)$ и $Y=\left(\mathbf{q}_{2}, \mathbf{v}_{2}\right)$ - две близкие фазовые точки, исходящие из одного рассеивателя и такие, что их образы под действием отображения столкновений попадают на тот же самый рассеиватель. В силу элементарной геометрии имеем

$$
\left\|\boldsymbol{\Delta}_{\varepsilon}(X)-\boldsymbol{\Delta}_{\varepsilon}(Y)\right\| \leqslant C \sqrt{\varkappa_{\min }^{-1}\left\|\boldsymbol{\Delta}_{\varepsilon}(X)\right\| \cdot \operatorname{dist}(X, Y)} .
$$

Отсюда следует, что эта оценка применима к биллиарду в области $\mathscr{Q}=T(\widetilde{\mathscr{D}})$. Легко видеть, что $\varkappa_{\min } \sim e^{-\varepsilon \Delta_{\varepsilon, x}}$, так что вышеприведенная оценка будет иметь множитель $e^{\varepsilon \Delta_{\varepsilon, x} / 2}$. Но когда мы преобразуем образы $X$ и $Y$ обратно в $\widetilde{\mathscr{D}}$, то расстояние между ними уменьшится с коэффициентом $e^{-\varepsilon \Delta_{\varepsilon, x}}$, который сократится с $e^{\varepsilon \Delta_{\varepsilon, x} / 2}$. Это доказывает лемму.

Таким образом, компоненты $\Delta_{\varepsilon, x}, \Delta_{\varepsilon, y}$ вектора $\boldsymbol{\Delta}_{\varepsilon}$ в ограничении на каждую ячейку гёльдеровски непрерывны в обычном смысле с гёльдеровским показателем $=1 / 2$ и с гёльдеровской нормой

$$
K_{\varepsilon}=\max _{X \in \mathscr{M}}\left\|\boldsymbol{\Delta}_{\varepsilon}(X)\right\| \sim \frac{C}{\sqrt{\varepsilon}}
$$

(см. (5.6)). Отсюда следует, что $\Delta_{\varepsilon, x}, \Delta_{\varepsilon, y}$ лежат в $\mathscr{H}$ с фиксированным значением параметра $\vartheta_{\Delta}<1$ (не зависящим от $\varepsilon$ ) и с $K_{\Delta} \sim 1 / \sqrt{\varepsilon}$. Заметим также, что

$$
\left\|\Delta_{\varepsilon, x}\right\|_{\infty} \sim\left\|\Delta_{\varepsilon, y}\right\|_{\infty} \sim \frac{1}{\sqrt{\varepsilon}} .
$$

Таким образом, применимы формулы типа Кавасаки (7.5), (7.6), которые дают

$$
\left|\int_{\mathscr{M}} \Delta \circ \mathscr{F}_{\varepsilon}^{n} d \nu_{0}-\int_{\mathscr{M}} \Delta d \nu_{\varepsilon}\right| \leqslant C \varepsilon^{-1 / 2} \theta^{n},
$$

где $\Delta-$ сокращенное обозначение для $\Delta_{\varepsilon, x}$ и $\Delta_{\varepsilon, y}$. Более того, для любого $k \geqslant 2$ имеем $\left\|\Delta^{k}\right\| \sim \varepsilon^{-k / 2}$ и

$$
\left|\int_{\mathscr{M}} \Delta^{k} \circ \mathscr{F}_{\varepsilon}^{n} d \nu_{0}-\int_{\mathscr{M}} \Delta^{k} d \nu_{\varepsilon}\right| \leqslant C \varepsilon^{-k / 2} \theta^{n} .
$$

При $k=1$ можно получить равномерную оценку, не зависящую от $\varepsilon$.

ПреДЛОЖЕНИЕ 8.3. Для некоторых констант $C>0 u \theta<1$, не зависящих от $\varepsilon$, выполняется неравенство

$$
\left|\int_{\mathscr{M}} \Delta \circ \mathscr{F}_{\varepsilon}^{n} d \nu_{0}-\int_{\mathscr{M}} \Delta d \nu_{\varepsilon}\right| \leqslant C \theta^{n},
$$

где $\Delta$ - сокращенное обозначение для $\Delta_{\varepsilon, x} u \Delta_{\varepsilon, y}$. 
ДоказАтельство. Определим $\Delta^{\prime}=\Delta \cdot \mathbf{1}_{\{|\Delta|<H\}}$ и $\Delta^{\prime \prime}=\Delta-\Delta^{\prime}$, где $H-$ некоторое "отсекающее" значение, которое будет выбрано ниже. Применение нашего предыдущего анализа к $\Delta^{\prime}$ показывает, что эта функция динамически гёльдеровски непрерывна с параметрами $\vartheta_{\Delta}<1$ (равномерным по $\varepsilon$ ) и $K_{H} \sim H$. Таким образом, из (7.3) следует неравенство

$$
\left|\int_{\mathscr{M}} \Delta^{\prime} \circ \mathscr{F}_{\varepsilon}^{n} d \nu_{0}-\int_{\mathscr{M}} \Delta^{\prime} d \nu_{\varepsilon}\right| \leqslant C H \theta^{n}
$$

для некоторых абсолютных констант $C>0$ и $\theta<1$.

Теперь для любого $n \geqslant 0$ имеем

$$
\left|\int_{\mathscr{M}} \Delta^{\prime \prime} \circ \mathscr{F}_{\varepsilon}^{n} d \nu_{0}\right| \leqslant \frac{C}{H}
$$

см. ниже. Переход к пределу при $n \rightarrow \infty$ дает $\left|\int_{\mathscr{M}} \Delta^{\prime \prime} d \nu_{\varepsilon}\right| \leqslant C / H$ и потому

$$
\left|\int_{\mathscr{M}} \Delta^{\prime \prime} \circ \mathscr{F}_{\varepsilon}^{n} d \nu_{0}-\int_{\mathscr{M}} \Delta^{\prime \prime} d \nu_{\varepsilon}\right| \leqslant \frac{2 C}{H}
$$

Для доказательства (8.6) заметим, что $\Delta \sim C m$ на любой ячейке $D_{m}^{(\mathrm{U})}$ (см. раздел 5$)$ для некоторого $C>0$, и, используя первую оценку в $(8.1)$, получим сумму

$$
\sum_{m=H / C}^{m_{\mathrm{U}}} \frac{C m}{m^{3}} \sim \frac{C}{H}
$$

Аналогично, $\Delta \sim C m$ на любой ячейке $D_{m}^{(\mathrm{L})}$, и, используя вторую оценку в $(8.1)$, получаем сумму

$$
\sum_{m=H / C}^{m_{L}} \frac{C m^{2}}{m_{\mathrm{L}}^{4}} \leqslant \frac{C}{m_{\mathrm{L}}} \leqslant \frac{C}{H}
$$

в случае $H<C m_{\mathrm{L}}$, а в противном случае сумма пустая.

Сочетание (8.5) и (8.7) дает, что для некоторого $C>0$

$$
\left|\int_{\mathscr{M}} \Delta \circ \mathscr{F}_{\varepsilon}^{n} d \nu_{0}-\int_{\mathscr{M}} \Delta d \nu_{\varepsilon}\right| \leqslant C\left(H \theta^{n}+1 / H\right) .
$$

Выбирая теперь $H=\theta^{-n / 2}$, получаем искомую равномерную оценку хвоста (8.4). Предложение 8.3 доказано.

Завершим этот раздел анализом “остаточной” функции, введенной в (4.4):

$$
\mathscr{R}_{\varepsilon}=\frac{1-e^{-\varepsilon \Delta_{\varepsilon, x}}-\varepsilon \Delta_{\varepsilon, x}}{\varepsilon}=\sum_{k=1}^{\infty} \frac{(-1)^{k}}{(k+1) !} \varepsilon^{k} \Delta_{\varepsilon, x}^{k+1} .
$$

Легко видеть, что $\mathscr{R}_{\varepsilon}$ динамически гёльдеровски непрерывна с теми же характеристиками $\vartheta_{\mathscr{R}_{\varepsilon}}=\vartheta_{\Delta}$ и $K_{\mathscr{R}_{\varepsilon}}=K_{\Delta}$, что и функция $\Delta_{\varepsilon, x}$.

С другой стороны, так как $\left|\Delta_{\varepsilon, x}\right|=\mathscr{O}\left(\varepsilon^{-1 / 2}\right)$, то функция $\mathscr{R} \varepsilon$ ограничена (равномерно по $\varepsilon$ ), т. е.

$$
\left|\mathscr{R}_{\varepsilon}\right| \leqslant C .
$$


Кроме того, $\mathscr{R}_{\varepsilon} \rightarrow 0$ поточечно при $\varepsilon \rightarrow 0$ (см. [1; с. 585]). По всем этим причинам работать с ее вкладом в (4.5) будет гораздо легче, чем с вкладом $\Delta_{\varepsilon, x}$; поэтому мы сначала сосредоточимся на основной сумме

$$
\sum_{k=1}^{\infty} \nu_{0}\left[\left(\Delta \circ \mathscr{F}_{\varepsilon}^{k}\right) \Delta_{\varepsilon, x}\right]
$$

в (4.5), а затем приспособим наши рассуждения для $\mathscr{R}_{\varepsilon}$. Сумма (8.10) состоит из похожих на корреляции членов, которые будут рассмотрены в следующем разделе.

\section{9. Оценки корреляций}

Перепишем (8.10) в виде

$$
\sum_{n=1}^{\infty} \nu_{0}\left[\left(\Delta_{\varepsilon} \circ \mathscr{F}_{\varepsilon}^{n}\right) \Delta_{\varepsilon}\right],
$$

где каждое $\Delta_{\varepsilon}$ можно заменить либо на $\Delta_{\varepsilon, x}$, либо на $\Delta_{\varepsilon, y}$, и наши оценки в этом разделе будут применимы ко всем четырем комбинациям (хотя мы имеем дело только с двумя в (4.5)). Кроме того, $\Delta_{0}$ будет означать смещение, когда $\varepsilon=0$, т. е. в условиях бесполевой (биллиардной) динамики (напомним, что $\Delta_{0}$ неограничена).

Сумма (9.1) содержит похожие на корреляции члены, но они не являются в точности корреляциями, так как мера $\nu_{0}$ не $\mathscr{F}_{\varepsilon}$-инвариантна. Чтобы пояснить наши идеи, сначала ограничим истинные корреляции для биллиардной функции смещения $\Delta_{0}$, что само по себе является новым результатом (он связан с недавними исследованиями в [2], но не был там сформулирован и не использовался).

ПРЕДЛОЖЕНИЕ 9.1. Для некоторых констант $C>0 u \theta \in(0,1)$ и для всех $n \geqslant 1$ выполняется неравенство

$$
\left|\nu_{0}\left[\left(\Delta_{0} \circ \mathscr{F}^{n}\right) \Delta_{0}\right]\right| \leqslant C \theta^{n},
$$

где каждое $\Delta_{0}$ можно заменить либо на $\Delta_{0, x}$, либо на $\Delta_{0, y}$ (под действием биллиардного отображения $\mathscr{F})$. Заметим, что ограничение $n \geqslant 1$ здесъ существенно, поскольку $\nu_{0}\left(\Delta_{0}^{2}\right)=\infty$.

ДокАЗАТЕЛЬСтво. Пространство столкновений $\mathscr{M}$ разделено кривыми разрыва отображения $\mathscr{F}$ на ячейки $D_{m}$ такие, что $\Delta_{0} \sim C m$ на $D_{m}$. Их меры удовлетворяют $\nu_{0}\left(D_{m}\right) \sim C / m^{3}$, их размерности являются $\sim C / \sqrt{m}$ в устойчивом направлении и $\sim C / m^{2}$ в неустойчивом направлении (в точности как "верхние" ячейки $D_{m}^{(\mathrm{U})}$ в нашем случае); это все стандартные факты [7], [25], [29], [2].

Так же, как в предыдущем разделе, пусть $\Delta_{0}^{\prime}=\Delta_{0} \cdot \mathbf{1}_{\left\{\left|\Delta_{0}\right|<H\right\}}$ и $\Delta_{0}^{\prime \prime}=\Delta_{0}-\Delta_{0}^{\prime}$, где $H$ - некоторое "отсекающее" значение, которое будет выбрано ниже. Положим также $\Delta_{0}^{\prime \prime \prime}=\Delta_{0} \cdot \mathbf{1}_{\left\{\left|\Delta_{0}\right|<H^{3}\right\}}$ и $\Delta_{0}^{\prime \prime \prime \prime}=\Delta_{0}-\Delta_{0}^{\prime \prime \prime}$. Как показано ранее, $\Delta_{0}^{\prime}$ и $\Delta_{0}^{\prime \prime \prime}$ динамически гёльдеровски непрерывны с параметрами $\vartheta_{\Delta}<1$ и соответственно $K_{H}=\mathscr{O}(H)$ и $K_{H^{3}}=\mathscr{O}\left(H^{3}\right)$. Таким образом, из стандартной оценки 
корреляций [7; § 7.7] следует, что

$$
\left|\nu_{0}\left[\left(\Delta_{0}^{\prime \prime \prime} \circ \mathscr{F}^{n}\right) \Delta_{0}^{\prime}\right]\right| \leqslant C H^{4} \theta^{n}
$$

для некоторых $C>0$ и $\theta<1$ (заметим, что $\nu_{0}\left(\Delta_{0}\right)=\nu_{0}\left(\Delta_{0}^{\prime}\right)=\nu_{0}\left(\Delta_{0}^{\prime \prime}\right)=0$ в силу того, что наша динамика обладает симметрией относительно обращения времени). Далее имеем

$\left|\nu_{0}\left[\left(\Delta_{0}^{\prime \prime \prime \prime} \circ \mathscr{F}^{n}\right) \Delta_{0}^{\prime}\right]\right| \leqslant C \sum_{m=1}^{H} \sum_{k=H^{3}}^{\infty} m k \nu_{0}\left[D_{m} \cap \mathscr{F}^{-n}\left(D_{k}\right)\right] \leqslant C \sum_{m=1}^{H} \sum_{k=H^{3}}^{\infty} \frac{m k}{k^{3}} \leqslant \frac{C}{H}$.

Остается оценить величину

$$
\mathscr{A}:=\left|\nu_{0}\left[\left(\Delta_{0} \circ \mathscr{F}^{n}\right) \Delta_{0}^{\prime \prime}\right]\right| \leqslant C \sum_{m=H}^{\infty} \sum_{k=1}^{\infty} m k \nu_{0}\left[D_{m} \cap \mathscr{F}^{-n}\left(D_{k}\right)\right] .
$$

Чтобы оценить меру $\nu_{0}\left[D_{m} \cap \mathscr{F}^{-n}\left(D_{k}\right)\right]$, расслоим $D_{m}$ неустойчивыми кривыми $W \subset D_{m}$; они имеют длины $\sim C / m^{2}$. Образ $\mathscr{F}\left(D_{m}\right)$ является областью длины $\sim C m^{-1 / 2}$ в неустойчивом направлении, примыкающей к линии $\varphi= \pm \pi / 2$, ограничивающей пространство $\mathscr{M}$ (см., например, [7; $\$ 4.10])$. Таким образом, кривые $\mathscr{F}(W)$ делятся на куски границами полос однородности (6.1); эти куски имеют длины $\sim 1 / j^{3}$ при $j \geqslant C m^{1 / 4}$, а их прообразы на $W$ имеют длины $\sim 1 /\left(m j^{5}\right)$, так как эти куски сжимаются под действием отображения $\mathscr{F}^{-1}$ с коэффициентом $\sim m j^{2}$. Кривая $W$, оснащенная условной плотностью $\rho$, индуцированной мерой $\nu_{0}$, является стандартной парой $\ell=(W, \rho)$, а ее образ $\mathscr{F}(W)$, оснащенный индуцированной мерой, является стандартным семейством $\mathscr{G}_{\ell, 1}$, у которого $\mathscr{Z}$-значение (6.9) удовлетворяет неравенству

$$
\mathscr{Z}_{\mathscr{G}_{\ell, 1}} \leqslant C \sum_{j=C m^{1 / 4}}^{\infty} \frac{m^{2} j^{3}}{m j^{5}} \leqslant C m^{3 / 4} .
$$

Так как это применимо к любой кривой $W$ в нашем слоении ячейки $D_{m}$, то мера $\nu_{0}$, рассматриваемая как условная мера на $D_{m}$, представлена мерой $\mathrm{P}_{\mathscr{G}}$ на соответствующем стандартном семействе $\mathscr{G}$, для образа которого $\mathscr{G}_{1}=\mathscr{F}(\mathscr{G})$ выполняется $\mathscr{Z}_{\mathscr{G}_{1}}=\mathscr{O}\left(\mathrm{m}^{3 / 4}\right)$. В силу (6.10) также выполняется $\mathscr{Z}_{\mathscr{G}_{n}} \leqslant \mathrm{Cm}^{3 / 4}$ для всех $n \geqslant 1$. Поскольку каждая ячейка $D_{k}$ имеет длину $\sim C k^{-2}$ в неустойчивом направлении, имеем

$$
\nu_{0}\left[D_{m} \cap \mathscr{F}^{-n}\left(D_{k}\right)\right] \leqslant C \nu_{0}\left(D_{m}\right) \mathscr{Z}_{\mathscr{G}_{n}} k^{-2} \leqslant C m^{-9 / 4} k^{-2} .
$$

Также имеем

$$
\nu_{0}\left[D_{m} \cap \mathscr{F}^{-n}\left(D_{k}\right)\right] \leqslant \nu_{0}\left(D_{k}\right)=C k^{-3} .
$$

Объединяя (9.6) и (9.7), оцениваем (9.5) следующим образом:

$$
\begin{aligned}
\mathscr{A} & \leqslant C \sum_{m=H}^{\infty} \sum_{k=1}^{m^{3}} m k m^{-9 / 4} k^{-2}+C \sum_{m=H}^{\infty} \sum_{k=m^{3}}^{\infty} m k k^{-3} \\
& \leqslant C \sum_{m=H}^{\infty} m^{-5 / 4} \log m+C \sum_{m=H}^{\infty} m m^{-3} \leqslant \frac{C}{H^{1 / 5}} .
\end{aligned}
$$


Собирая теперь наши оценки (9.3), (9.4), (9.8) и выбирая $H=\theta^{-n / 5}$ (чтобы подавить множитель $H^{4}$ в $\left.(9.3)\right)$, получаем доказательство предложения 9.1.

Основные трудности в доказательстве вызваны неограниченностью функции $\Delta_{0}$. Если заменить ее на ограниченный вариант

$$
\Delta_{0}^{(H)}=\Delta_{0} \cdot \mathbf{1}_{\left\{\left|\Delta_{0}\right|<H\right\}}
$$

то доказательство легко модифицируется, и мы получаем следующее утверждение.

СлЕДСТвИЕ 9.2. Для некоторых констант $C>0 u \theta \in(0,1)$ u для всех $n \geqslant 1$ выполняется неравенство

$$
\left|\nu_{0}\left[\left(\Delta_{0}^{\left(H_{1}\right)} \circ \mathscr{F}^{n}\right) \Delta_{0}^{\left(H_{2}\right)}\right]\right| \leqslant C \theta^{n} .
$$

Снова заметим, что так как наша динамика допускает обращение времени, имеем

$$
\nu_{0}\left(\Delta_{0}^{(H)}\right)=0
$$

для любого $H>0$. Оценка (9.10) неверна при $n=0$. На самом деле имеем

$$
\nu_{0}\left(\left[\Delta_{0}^{(H)}\right]^{2}\right)=\mathscr{O}\left(\sum_{m=1}^{H} \frac{m^{2}}{m^{3}}\right)=\mathscr{O}(\log H) .
$$

Распространим предложение 9.1 на возмущенное отображение $\mathscr{F}_{\varepsilon}$.

ПрЕДЛОЖЕНИЕ 9.3. Для некоторых констант $C>0 u \theta \in(0,1)$, не зависящих от $\varepsilon$, и для всех $n \geqslant 1$ выполняются неравенства

$$
\begin{aligned}
& \left|\nu_{\varepsilon}\left[\left(\Delta_{\varepsilon} \circ \mathscr{F}_{\varepsilon}^{n}\right) \widetilde{\Delta}_{\varepsilon}\right]-\nu_{\varepsilon}\left(\Delta_{\varepsilon}\right) \nu_{\varepsilon}\left(\widetilde{\Delta}_{\varepsilon}\right)\right| \leqslant C \theta^{n}, \\
& \left|\nu_{0}\left[\left(\Delta_{\varepsilon} \circ \mathscr{F}_{\varepsilon}^{n}\right) \widetilde{\Delta}_{\varepsilon}\right]-\nu_{\varepsilon}\left(\Delta_{\varepsilon}\right) \nu_{0}\left(\widetilde{\Delta}_{\varepsilon}\right)\right| \leqslant C \theta^{n},
\end{aligned}
$$

где каждое из $\Delta_{\varepsilon}$ и $\widetilde{\Delta}_{\varepsilon}$ можно заменить либо на $\Delta_{\varepsilon, x}$, либо на $\Delta_{\varepsilon, y}$.

ДоказАтельство. Доказательство неравенства (9.13) очень похоже на доказательство предложения 9.1. "Верхние" ячейки $D_{m}^{(\mathrm{U})}$ обладают формой, размером и $\nu_{\varepsilon}$-мерой, заданными теми же формулами, что и для ячеек $D_{m}$ в бесполевом газе Лоренца. Теперь имеются "нижние" ячейки $D_{m}^{(\mathrm{L})}$, характеристики которых можно вычислить напрямую, и затем можно следовать схеме доказательства предложения 9.1, что несложно, пусть и весьма нетривиально технически, так что мы опускаем детали.

В том, что наше рассуждение работает, можно также убедиться с помощью следующего наблюдения. "Нижние" ячейки $D_{m}^{(\mathrm{L})}$ расположены в том месте в $\mathscr{M}$, где находятся обыкновенные ячейки $D_{k}$ для бесполевого газа Лоренца (ср. наш рис. 4 с рис. 4.15 в [7]), только наши "нижние" ячейки толще. Они также отображаются под действием $\mathscr{F}_{\varepsilon}$ так же, как обыкновенные ячейки $D_{k}$ отображаются под действием $\mathscr{F}$ (ср. наш рис. 5 с рис. 4.16 в [7]). Таким образом, можно измельчить "нижние" ячейки $D_{m}^{(\mathrm{L})}$, заменив каждую из них объединением более тонких областей той же формы, что обыкновенные ячейки $D_{k}$ (легко 
видеть, что $k$ должно принимать значения $m_{\mathrm{L}}^{2} / m \leqslant k<m_{\mathrm{L}}^{2} /(m-1)$, а последняя ячейка $D_{1}^{(\mathrm{L})}$ будет заменена счетным объединением ячеек $D_{k}$ со всеми индексами $k \geqslant m_{\mathrm{L}}^{2}$. Можно также переопределить отображение $\mathscr{F} \varepsilon$ на $D_{m}^{(\mathrm{L})}$ так, чтобы оно действовало как $\mathscr{F}$ на соответствующих ячейках $D_{k}$. Тогда можно будет применить наш анализ кривых $W$, расслаивающих ячейки $D_{k}$ (и тем самым также расслаивающих ячейки $\left.D_{m}^{(\mathrm{L})}\right)$, и получатся аналогичные оценки $\mathscr{Z}$-значений, как в доказательстве предложения 9.1. Единственная существенная разница состоит в том, что наши наблюдаемые величины $\Delta_{\varepsilon}$ существенно меньше, чем $\Delta_{0}$, поскольку $\Delta_{\varepsilon} \sim m$ на $D_{m}^{(\mathrm{L})}$, в то время как $\Delta_{0} \sim k$ на "заменяющих" ячейках $D_{k}$, и $k \gg m$. Таким образом, наши наблюдаемые величины $\Delta_{\varepsilon}$ вызывают гораздо меньше хлопот, чем $\Delta_{0}$, и все оценки, установленные в доказательстве предложения 9.1, останутся справедливыми.

Обратимся к доказательству неравенства (9.14). Поскольку мера $\nu_{0}$ не $\mathscr{F}_{\varepsilon}$-инвариантна, сначала нужно применить замечание 7.1 , а затем повторить вышеприведенное доказательство неравенства (9.13). Предложение 9.3 доказано.

Теперь вернемся к (4.5). В силу предложения 8.3 (см. также (7.5), (7.6)) ряд (4.5) сходится экспоненциально быстро и равномерно по $\varepsilon$, откуда следует, что

$$
\left|\sum_{n=L|\log \varepsilon|}^{\infty} \nu_{0}\left[\left(\Delta \circ \mathscr{F}_{\varepsilon}^{n}\right)(1-g)\right]\right|=\mathscr{O}\left(\varepsilon^{2}\right)
$$

для достаточно большого $L>0$, поэтому остается разобраться с $n<L|\log \varepsilon|$. Далее мы будем иметь дело с рядом в правой части из (4.5), т. е. мы отщепим множитель от $\varepsilon$.

Значения $\nu_{0}\left[\left(\Delta_{\varepsilon} \circ \mathscr{F}_{\varepsilon}^{n}\right) \Delta_{\varepsilon}\right]$ в (9.1) можно оценить с помощью неравенства (9.14) из предложения 9.3:

$$
\nu_{0}\left[\left(\Delta_{\varepsilon} \circ \mathscr{F}_{\varepsilon}^{n}\right) \Delta_{\varepsilon}\right]=\nu_{0}\left(\Delta_{\varepsilon}\right) \nu_{\varepsilon}\left(\Delta_{\varepsilon}\right)+\mathscr{O}\left(\theta^{n}\right),
$$

и аналогично, заменяя одно из $\Delta_{\varepsilon}$ на гораздо более слабую функцию $\mathscr{R}_{\varepsilon}$, получаем

$$
\nu_{0}\left[\left(\Delta_{\varepsilon} \circ \mathscr{F}_{\varepsilon}^{n}\right) \mathscr{R}_{\varepsilon}\right]=\nu_{0}\left(\mathscr{R}_{\varepsilon}\right) \nu_{\varepsilon}\left(\Delta_{\varepsilon}\right)+\mathscr{O}\left(\theta^{n}\right) .
$$

(Может показаться странным, что мы приближаем члены сходящегося ряда (9.1) двумя ненулевыми константами, но на самом деле

$$
\nu_{0}\left(\Delta_{\varepsilon}\right) \nu_{\varepsilon}\left(\Delta_{\varepsilon}\right)+\nu_{0}\left(\mathscr{R}_{\varepsilon}\right) \nu_{\varepsilon}\left(\Delta_{\varepsilon}\right)=0
$$

что следует из соотношения $\nu_{0}(1-g)=0$; ср. раздел 4.) Теперь вклад всех $\mathscr{O}\left(\theta^{n}\right)$-членов из $(9.15),(9.16)$ в сумму $(4.5)$ будет в совокупности являться $\mathscr{O}(\varepsilon)$, что приемлемо. Но вклад членов $\nu_{0}\left(\Delta_{\varepsilon}\right) \nu_{\varepsilon}\left(\Delta_{\varepsilon}\right)$ и $\nu_{0}\left(\mathscr{R}_{\varepsilon}\right) \nu_{\varepsilon}\left(\Delta_{\varepsilon}\right)$ нельзя оценить напрямую, так как он содержит неизвестную величину $\nu_{\varepsilon}\left(\Delta_{\varepsilon}\right)$. Чтобы обойти это осложнение, заметим, что

$$
\nu_{0}\left(\Delta_{\varepsilon}\right)=\mathscr{O}\left(\sum_{m=1}^{m_{\mathrm{U}}} \frac{m}{m^{3}}\right)+\mathscr{O}\left(\sum_{m=1}^{m_{\mathrm{L}}} \frac{m^{2}}{m_{\mathrm{L}}^{4}}\right)=\mathscr{O}(1) .
$$


(На самом деле, мы полагаем, что более тщательная оценка дала бы $\nu_{0}\left(\Delta_{\varepsilon}\right)=$ $\mathscr{O}(\sqrt{\varepsilon})$, но здесь это нам не потребуется.) Далее, $\nu_{0}\left(\mathscr{R}_{\varepsilon}\right)=\mathscr{O}(1)$, так как $\mathscr{R}_{\varepsilon}$ равномерно ограничена в силу (8.9). Таким образом, можно переписать (4.3) и (4.5) как

$$
\nu_{\varepsilon}\left(\Delta_{\varepsilon}\right)=\frac{1}{2} \nu_{0}\left[\Delta_{\varepsilon}(1-g)\right]+L \varepsilon|\log \varepsilon|\left(\nu_{0}\left(\Delta_{\varepsilon}\right)+\nu_{0}\left(\mathscr{R}_{\varepsilon}\right)\right) \nu_{\varepsilon}\left(\Delta_{\varepsilon}\right)+\mathscr{O}(\varepsilon) .
$$

Решение этого уравнения относительно $\nu_{\varepsilon}\left(\Delta_{\varepsilon}\right)$ дает

$$
\nu_{\varepsilon}\left(\Delta_{\varepsilon}\right)=\frac{1}{2} \nu_{0}\left[\Delta_{\varepsilon}(1-g)\right](1+\mathscr{O}(\varepsilon|\log \varepsilon|))+\mathscr{O}(\varepsilon)
$$

Остается подсчитать $\nu_{0}\left[\Delta_{\varepsilon}(1-g)\right]$ и доказать предложение 3.1 , что будет сделано в следующем разделе.

\section{0. Окончание доказательства теоремы 9}

Здесь мы докажем остальные формулы (4.6)-(4.8) и (4.13).

Чтобы проверить (4.6), заметим, что

$$
\nu_{0}\left(\Delta_{\varepsilon, x}^{k+2}\right)=\mathscr{O}\left(\sum_{m=1}^{m_{\mathrm{U}}} \frac{m^{k+2}}{m^{3}}\right)+\mathscr{O}\left(\sum_{m=1}^{m_{\mathrm{L}}} \frac{m^{k+3}}{m_{\mathrm{L}}^{4}}\right)=\mathscr{O}\left(\varepsilon^{-k / 2}\right)
$$

для любого $k \geqslant 1$. Затем использование ряда (8.8) дает

$$
\nu_{0}\left(\Delta_{\varepsilon, x} \mathscr{R}_{\varepsilon}\right)=\mathscr{O}\left(\sum_{k=1}^{\infty} \frac{\varepsilon^{k / 2}}{(k+1) !}\right)=\mathscr{O}\left(\varepsilon^{1 / 2}\right) .
$$

Что же касается (4.7), то имеем

$$
\nu_{0}\left(\Delta_{\varepsilon, x}^{2}\right)=\mathscr{O}\left(\sum_{m=1}^{m_{\mathrm{U}}} \frac{m^{2}}{m^{3}}\right)+\mathscr{O}\left(\sum_{m=1}^{m_{\mathrm{L}}} \frac{m^{3}}{m_{\mathrm{L}}^{4}}\right)=\mathscr{O}(|\log \varepsilon|)+\mathscr{O}(1),
$$

и аналогичная оценка без труда применима к $\nu_{0}\left(\Delta_{\varepsilon, x} \Delta_{\varepsilon, y}\right)$. Чтобы получить более точные асимптотики, которые утверждаются в $(4.7),(4.8)$, нужно вернуться к геометрическому анализу из раздела 5 .

Во-первых, заметим, что вклад из нижних ячеек $D_{m}^{(\mathrm{L})}$ является $\mathscr{O}(1)$ согласно (10.2), откуда следует, что ими можно пренебречь. Во-вторых, можно выбрать большую константу $M \gg 1$ и пренебречь всеми фазовыми точками с $\left\|\boldsymbol{\Delta}_{\varepsilon}\right\| \leqslant M$. Итак, мы рассматриваем только траектории, которые выходят с начального рассеивателя в сторону бесконечного коридора (см. $B_{1}$ на рис. 6) и попадают на удаленные рассеиватели на противоположной стороне этого коридора (верхний ряд на рис. 6).

Далее обозначим через $L_{1}$ и $L_{2}$ две параллельные прямые, ограничивающие наш коридор, т. е. две общие касательные ко всем рассеивателям вдоль нашего коридора (см. рис. 6). Наши траектории покидают рассеиватель $B_{1}$, сначала пересекают $L_{1}$, затем после долгого пути в коридоре они пересекают $L_{2}$ и очень скоро попадают на следующий рассеиватель в верхнем ряду. Обозначим через 


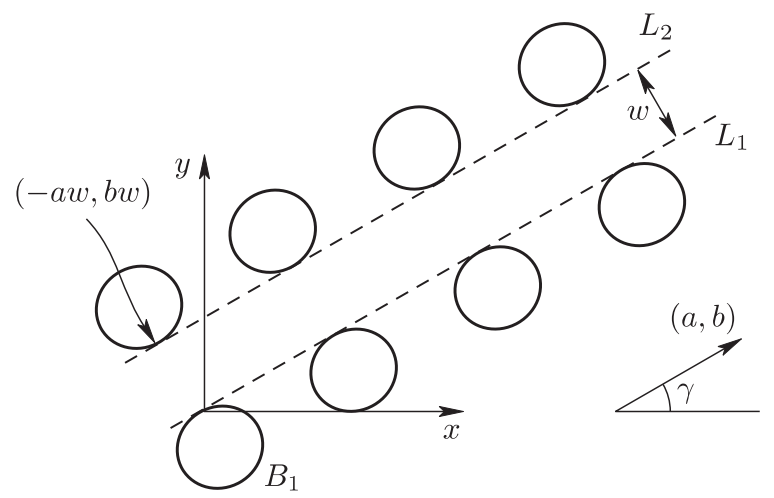

Рис. 6. Бесконечный коридор, ограниченный двумя прямыми, касающимися рассеивателей

$\widehat{\boldsymbol{\Delta}}_{\varepsilon}=\left(\widehat{\Delta}_{\varepsilon, x}, \widehat{\Delta}_{\varepsilon, y}\right)$ вектор между двумя точками пересечения соответственно на $L_{1}$ и на $L_{2}$. Заметим, что $\Delta_{\varepsilon, x}=\widehat{\Delta}_{\varepsilon, x}+\mathscr{O}(1)$ и $\Delta_{\varepsilon, y}=\widehat{\Delta}_{\varepsilon, y}+\mathscr{O}(1)$, так что

$$
\nu_{0}\left(\Delta_{\varepsilon, x}^{2}\right)=\nu_{0}\left(\widehat{\Delta}_{\varepsilon, x}^{2}\right)+\mathscr{O}(1), \quad \nu_{0}\left(\Delta_{\varepsilon, x} \Delta_{\varepsilon, y}\right)=\nu_{0}\left(\widehat{\Delta}_{\varepsilon, x} \widehat{\Delta}_{\varepsilon, y}\right)+\mathscr{O}(1),
$$

и потому можно заменить $\Delta_{\varepsilon, x}, \Delta_{\varepsilon, y}$ на $\widehat{\Delta}_{\varepsilon, x}, \widehat{\Delta}_{\varepsilon, y}$.

Снова воспользуемся преобразованием $T$, которое выпрямляет все наши траектории и преобразует область $\widetilde{\mathscr{D}}$ в $\mathscr{Q}=T(\widetilde{\mathscr{D}})$. Прямые $L_{1}$ и $L_{2}$ отображаются в кривые $T\left(L_{1}\right)$ и $T\left(L_{2}\right)$ на $u v$-плоскости (см. рис. 7$)$. Выберем систему координат так, чтобы линия $L_{1}$ проходила через начало координат, и обозначим, как и прежде, ширину коридора через $w$, а угол между коридором и направлением поля (т. е. осью $x$ ) через $\gamma$. Тогда вторая линия $L_{2}$ будет задана параметрическими уравнениями $x=a s-b w$ и $y=b s+a w$, где $a=\cos \gamma$ и $b=\sin \gamma$ (заметим, что $s$ - параметр длины дуги на $\left.L_{1}\right)$. Кривая $T\left(L_{2}\right)$ теперь задается параметрическими уравнениями

$$
\begin{aligned}
& u=\varepsilon^{-1}\left[e^{\varepsilon(a s-b w)} \cos \varepsilon(b s+a w)-1\right], \\
& v=\varepsilon^{-1} e^{\varepsilon(a s-b w)} \sin \varepsilon(b s+a w) .
\end{aligned}
$$

Напомним, что коэффициент растяжения преобразования $T$ равен $e^{\varepsilon x}=1+$ $\mathscr{O}(\sqrt{\varepsilon})$, откуда следует, что $\widehat{\Delta}_{\varepsilon, x}=u+\mathscr{O}(1)$ и $\widehat{\Delta}_{\varepsilon, y}=v+\mathscr{O}(1) ;$ отсюда следует, что можно далее заменить $\widehat{\Delta}_{\varepsilon, x}, \widehat{\Delta}_{\varepsilon, y}$ на $u, v$. В последующем вычислении $s$ будет параметризовать пересечения наших траекторий с $L_{1}$ и $L_{2}$.

Обозначим через $\theta$ угол между (прямой) траекторией на $u v$-плоскости и горизонтальной осью $u$. Обозначим через $D$ расстояние между последовательными рассеивателями, касающимися прямой $L_{1}$. Наши траектории тогда начинаются на отрезке длины $D$ на прямой $L_{1}$ и образуют с ней угол $\varphi=\theta-\gamma$. Обозначим этот отрезок через $\widehat{L}_{1}$. Его можно рассматривать как "искусственную" часть границы нашего биллиардного стола $\mathscr{Q}$, и тогда образ меры $\nu_{0}$ на нем был бы гладкой мерой с плотностью

$$
c_{\nu} \cos \varphi d r d \varphi=c_{\nu} \sin (\theta-\gamma) d r d \theta
$$




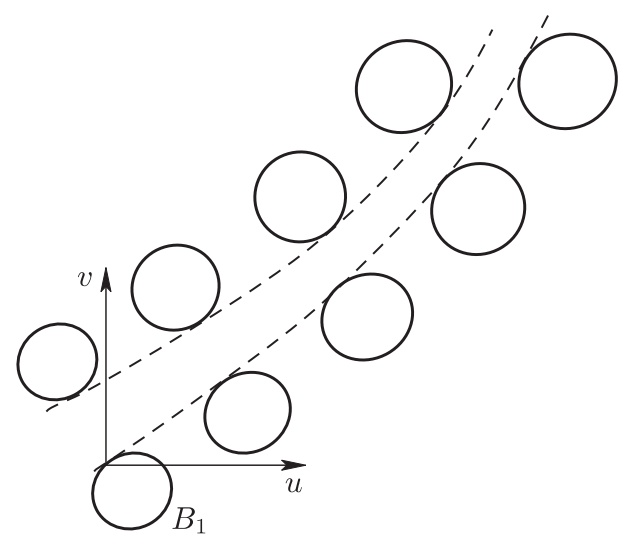

Рис. 7. Преобразованный бесконечный коридор на $u v$-плоскости, ограниченный двумя кривыми, касающимися рассеивателей

где $r$ - параметр длины на $\widehat{L}_{1}$, а $\varphi=\pi / 2-(\theta-\gamma)-$ угол, образованный выходящими траекториями с нормалью к $\widehat{L}_{1}(\mathrm{cp} .(1.1))$. Теперь вклад в $\nu_{0}\left(\Delta_{\varepsilon, x}^{2}\right)$ всех рассмотренных выше траекторий будет равен

$$
\mathscr{I}=c_{\nu} D \int_{s_{\min }}^{s_{\max }} u^{2} \sin (\theta-\gamma) d \theta+\mathscr{O}(1)
$$

для некоторых $s_{\min }=\mathscr{O}(1)$ и $s_{\max } \sim C / \sqrt{\varepsilon}$. Чтобы рассмотреть $\nu_{0}\left(\Delta_{\varepsilon, x} \Delta_{\varepsilon, y}\right)$, просто заменим $u^{2}$ на $u v$ в (10.4).

Теперь в силу элементарной геометрии имеем

$$
\sin (\theta-\gamma)=\frac{a v-b u+\mathscr{O}(1)}{\left[u^{2}+v^{2}\right]^{1 / 2}}
$$

а также

$$
d \theta=\frac{v \frac{d u}{d s}-u \frac{d v}{d s}+\mathscr{O}(1)}{u^{2}+v^{2}} d s=\left[\frac{a v-b u+\mathscr{O}(1)}{u^{2}+v^{2}}-\varepsilon b\right] d s
$$

Эти формулы легко вывести в предположении, что начальная точка есть $(0,0)$; тогда член $\mathscr{O}(1)$ не нужен, он отвечает только за изменение начальной точки на отрезке $\widehat{L}_{1}$ длины $D=\mathscr{O}(1)$.

Теперь использование разложения Тейлора дает следующее: $u=a s+\mathscr{O}(1)$, $v=b s+\mathscr{O}(1), u^{2}+v^{2}=s^{2}+\mathscr{O}(s)$ и

$$
a v-b u=w+\frac{1}{2} b \varepsilon s^{2}+\mathscr{O}(\sqrt{\varepsilon}) .
$$

Подстановка этих формул в (10.4) и интегрирование от 0 до $s_{\max } \sim C / \sqrt{\varepsilon}$ без труда дает

$$
\mathscr{I}=\frac{1}{2} c_{\nu} a^{2} w^{2} D|\log \varepsilon|+\mathscr{O}(1)
$$


а для $\nu_{0}\left(\Delta_{\varepsilon, x} \Delta_{\varepsilon, y}\right)$ мы просто заменяем $a^{2}$ на $a b$. Остается просуммировать по всем бесконечным коридорам и по всем отрезкам между последовательными рассеивателями на их границах. Тогда получим в точности

$$
\begin{aligned}
\nu_{0}\left(\Delta_{\varepsilon, x}^{2}\right) & =\widehat{\mathbf{D}}_{x, x}|\log \varepsilon|+\mathscr{O}(1), \\
\nu_{0}\left(\Delta_{\varepsilon, x} \Delta_{\varepsilon, y}\right) & =\widehat{\mathbf{D}}_{x, y}|\log \varepsilon|+\mathscr{O}(1),
\end{aligned}
$$

где через $\widehat{\mathbf{D}}$... обозначены соответствующие компоненты матрицы супердиффузии $\widehat{\mathbf{D}}$, заданной в (2.1). Это завершает доказательство соотношений (4.6)-(4.8).

Далее докажем (3.8), (3.9), следуя нашему плану, намеченному в разделе 4. Во-первых, проверим, что сходимость ряда (4.9), за исключением члена $n=0$, экспоненциальная и равномерная по $\varepsilon$. Это следует из (9.13) в предложении 9.3. Во-вторых, уже установлено, что $\nu_{\varepsilon}\left(\boldsymbol{\Delta}_{\varepsilon}\right)=\mathscr{O}(1)$ (см., например, (9.17)).

Остается ограничить ряд

$$
\sum_{k=1}^{\infty} \nu_{0}\left[\left(\Delta_{\varepsilon, x}^{2} \circ \mathscr{F}_{\varepsilon}^{k}\right)(1-g)\right]=\varepsilon \sum_{n=1}^{\infty} \nu_{0}\left[\left(\Delta_{\varepsilon, x}^{2} \circ \mathscr{F}_{\varepsilon}^{n}\right)\left(\Delta_{\varepsilon, x}+\mathscr{R}_{\varepsilon}\right)\right],
$$

появляющийся в (4.11). Применим то же рассуждение, что для (9.15), (9.16). Прежде всего, в силу (8.3) имеем

$$
\sum_{k=n}^{\infty} \nu_{0}\left[\left(\Delta_{\varepsilon, x}^{2} \circ \mathscr{F}_{\varepsilon}^{k}\right)(1-g)\right] \leqslant C \varepsilon^{-1} \theta^{n}
$$

для всех $n \geqslant 1$, с константой $\theta<1$. Полагая $n=L|\log \varepsilon|$ с достаточно большим $L>0$, получаем для хвоста оценку $\mathscr{O}(1)$, так что остается рассмотреть $n<$ $L|\log \varepsilon|$. С этой целью воспользуемся неравенством Гёльдера:

$$
\left|\nu_{0}\left[\left(\Delta_{\varepsilon, x}^{2} \circ \mathscr{F}_{\varepsilon}^{n}\right)\left(\Delta_{\varepsilon, x}+\mathscr{R}_{\varepsilon}\right)\right]\right| \leqslant\left[\left(\nu_{0} \circ \mathscr{F}_{\varepsilon}^{n}\right)\left(\left|\Delta_{\varepsilon, x}^{3}\right|\right)\right]^{2 / 3}\left[\nu_{0}\left(\left|\Delta_{\varepsilon, x}+\mathscr{R}_{\varepsilon}\right|^{3}\right)\right]^{1 / 3},
$$

причем каждый из этих интегралов не превосходит

$$
C \sum_{m=1}^{m_{\mathrm{U}}} \frac{m^{3}}{m^{3}}+C \sum_{m=1}^{m_{\mathrm{L}}} \frac{m^{4}}{m_{\mathrm{L}}^{4}} \leqslant C\left(m_{\mathrm{U}}+m_{\mathrm{L}}\right) \leqslant \frac{C}{\sqrt{\varepsilon}}
$$

(вспомним лемму 8.1 и доказательство предложения 8.3). Суммирование по $n \leqslant$ $L|\log \varepsilon|$ дает

$$
\varepsilon \sum_{n=1}^{L|\log \varepsilon|} \nu_{0}\left[\left(\Delta_{\varepsilon, x}^{2} \circ \mathscr{F}_{\varepsilon}^{n}\right)\left(\Delta_{\varepsilon, x}+\mathscr{R}_{\varepsilon}\right)\right] \leqslant C \sqrt{\varepsilon}|\log \varepsilon|,
$$

что завершает доказательство теоремы 9.

Обратимся к доказательству предложения 3.1, следуя нашему плану в конце раздела 4. Сначала можно напрямую проверить, что функция времени столкновения $\tau_{\varepsilon}$ обладает всеми теми же свойствами, что и функция смещения $\Delta_{\varepsilon}$, откуда следует, что ряд (4.12) сходится экспоненциально и равномерно по $\varepsilon$. Поэтому достаточно доказать (4.13), а это требует геометрического анализа.

В силу (5.1) легко видеть, что если траектория начинается из фазовой точки $(\mathbf{q}, \mathbf{v})$ и движется без столкновений в течение времени $\tau$, то она отклоняется 
от биллиардной траектории, начинающейся в той же фазовой точке, на $\mathscr{O}\left(\varepsilon \tau^{2}\right)$. Итак, на большей части пространства $\mathscr{M}$ имеем $\left|\tau_{\varepsilon}-\tau\right|=\mathscr{O}(\varepsilon)$. Единственными исключениями являются

(i) $\mathscr{O}(\varepsilon)$-окрестность линий разрыва (где образы точек под действием $\mathscr{F}$ и $\mathscr{F}_{\varepsilon}$ могут попасть на различные рассеиватели, так что $\left.\left|\tau_{\varepsilon}-\tau\right|=\mathscr{O}(1)\right)$,

и

(ii) ячейки $D_{m}, D_{m}^{(\mathrm{U})}$ и $D_{m}^{(\mathrm{L})}$ (описанные ранее), на которых велико либо $\tau$, либо $\tau_{\varepsilon}$ соответственно.

Пусть $b>0$ - малая константа. Вклад в (4.13) из всех ячеек $D_{m}, D_{m}^{(\mathrm{U})}$ с индексами $m \geqslant \varepsilon^{-b}$ не будет превосходить

$$
C \sum_{m=\varepsilon^{-b}}^{\infty} \frac{m}{m^{3}}=C \varepsilon^{b},
$$

так как $\tau \sim C m$ на $D_{m}$ и $\tau_{\varepsilon} \sim C m$ на $D_{m}^{(\mathrm{U})}$; поэтому все такие ячейки можно отбросить. "Нижние" ячейки $D_{m}^{(\mathrm{L})}$ можно полностью отбросить, так как

$$
\sum_{m=1}^{m_{\mathrm{L}}} \frac{m^{2}}{m_{\mathrm{L}}^{4}}=\mathscr{O}\left(m_{\mathrm{L}}^{-1}\right)=\mathscr{O}\left(\varepsilon^{1 / 2}\right) .
$$

Пусть $X \in D_{m}^{(\mathrm{U})}$. Если образы точки $X$ под действием отображений $\mathscr{F}_{\varepsilon}$ и $\mathscr{F}$ попадают на один и тот же рассеиватель, то они будут на расстоянии $\mathscr{O}(m \sqrt{\varepsilon})$ друг от друга, так как их траектории будут пробегать $\mathscr{O}\left(m^{2} \varepsilon\right)$-близко. Отсюда следует, что такие точки вносят суммарный вклад

$$
C \sum_{m=1}^{\varepsilon^{-b}} \frac{m \sqrt{\varepsilon}}{m^{3}} \leqslant C \sqrt{\varepsilon} .
$$

Если образы $X \in D_{m}^{(U)}$ под действием отображений $\mathscr{F} \varepsilon$ и $\mathscr{F}$ попадают на различные рассеиватели, то один из них попадает в $\mathscr{O}(m \sqrt{\varepsilon})$-окрестность края пространства $\mathscr{M}$ (определяемого соотношением $\varphi= \pm \pi / 2)$, и потому $X$ должна быть в $\mathscr{O}(\sqrt{\varepsilon})$-окрестности границы ячейки $D_{m}^{(\mathrm{U})}$ (так как коэффициент растяжения не меньше $C m$ ). При $m<\varepsilon^{-b}$ ширина ячеек $D_{m}^{(\mathrm{U})}$ и $D_{m}$ является $\sim C m^{-2} \gg \sqrt{\varepsilon}$, поэтому $X$ лежит в $D_{m^{\prime}}$ с индексом $m^{\prime}=m \pm 1$, т. е. $\left|\tau_{\varepsilon}-\tau_{0}\right|=\mathscr{O}(1)$. Таким образом, вклад всех таких точек равен

$$
C \sum_{m=1}^{\varepsilon^{-b}} \frac{\sqrt{\varepsilon}}{m} \leqslant C \sqrt{\varepsilon}|\log \varepsilon| .
$$

Сочетание всех наших оценок доказывает (4.13), что и завершает доказательство предложения 3.1 .

\section{1. Доказательство теоремы 8}

Здесь мы докажем теорему 8 о супердиффузии в свободных газах Лоренца с дискретным временем по модулю некоторых технических оценок моментов, которые будут завершены в следующем разделе. Начнем с части (а), а затем опишем изменения, нужные для части (б). 
Напомним, что

$$
\widetilde{\mathbf{q}}_{n}-\widetilde{\mathbf{q}}_{0}=\boldsymbol{\Delta}_{0}+\boldsymbol{\Delta}_{1}+\cdots+\boldsymbol{\Delta}_{n-1},
$$

где $\boldsymbol{\Delta}_{j}=\widetilde{\mathbf{q}}_{j+1}-\widetilde{\mathbf{q}}_{j}$ обозначает вектор смещения между последовательными столкновениями. Сначала обрежем неограниченную функцию $\boldsymbol{\Delta}$ следующим образом:

$$
\widehat{\Delta}_{j}=\boldsymbol{\Delta}_{j} \mathbf{1}_{\left\{\left\|\Delta_{j}\right\|<R\right\}}, \quad R=\frac{\sqrt{n}}{\log ^{100} n} .
$$

Лемма 11.1. Имеет место следующая сходимость по вероятности:

$$
\max _{m \leqslant n} \frac{\widetilde{\mathbf{q}}_{m}-\sum_{j=0}^{m-1} \widehat{\boldsymbol{\Delta}}_{j}}{\sqrt{n \log n}} \rightarrow 0 .
$$

Эта и последующие леммы будут доказаны в следующем разделе. Продолжим доказательство теоремы 8. Лемма 11.1 позволяет заменить неограниченную функцию $\Delta$ на ее обрезанную версию $\widehat{\Delta}$.

Далее мы будем пользоваться стандартным методом Бернштейна, основанном на технике "больших и малых блоков". Это означает, что мы делим временной интервал $[1, n]$ на большие блоки длины $N \sim n^{0.6}$, перемежающиеся с малыми блоками длины $\sim n^{0.01}$ (начиная с малого блока). Соответственно пусть

$$
\mathbf{Q}_{k}=\sum_{j=\left(n^{0.01}+N\right)(k-1)}^{n^{0.01} k+N(k-1)-1} \widehat{\Delta}_{j}, \quad \mathbf{Z}_{k}=\sum_{n^{0.01}}^{j=\left(n^{0.01}+N\right) k-1} \widehat{\Delta}_{j} .
$$

Таким образом, $\mathbf{Z}_{k}$ - суммы по большим блокам, а $\mathbf{Q}_{k}$ - суммы по малым блокам.

Лемма 11.2. Имеет место следующая сходимость по вероятности:

$$
\frac{\sum_{k} \mathbf{Q}_{k}}{\sqrt{n \log n}} \rightarrow 0
$$

Таким образом, достаточно рассмотреть только большие блоки. Они отделены друг от друга пробелами (состоящими из малых блоков), которые делают суммы $\mathbf{Z}_{k}$ в различных больших блоках почти независимыми. Уточним это утверждение: пусть

$$
m_{k}^{\prime}=\left(n^{0.01}+N\right) k+\frac{1}{3} n^{0.01}
$$

- момент времени внутри малого блока спустя $\frac{1}{3} n^{0.01}$ столкновений после предыдущего большого блока. Меру $\nu_{0}$ можно представить в виде Р $\mathscr{G}_{0}$ для собственного стандартного семейства (см. конец раздела 6). Прообразы кривых $W \in \mathscr{G}_{0}$ экспоненциально малы в момент времени $\left(n^{0.01}+N\right) k$, т. е. в конце предыдущего большого блока; отсюда следует, что функцию $\widehat{\Delta}$, измеренную в моменты времени $j \leqslant\left(n^{0.01}+N\right) k$, на таких кривых можно заменить на константу с точностью до экспоненциально малой (точнее, $\left.\mathscr{O}\left(\theta^{n^{0.01}} R\right)\right)$ погрешности. Теперь в момент времени

$$
m_{k}^{\prime \prime}=\left(n^{0.01}+N\right) k+\frac{2}{3} n^{0.01}
$$


все кривые $W \in \mathscr{G}_{0}$ длины $\geqslant \theta^{\frac{1}{3} n^{0.01}}$ вырастут в собственные семейства (кривые длины $<\theta^{\frac{1}{3} n^{0.01}}$ образуют множество экспоненциально малой меры). Затем возьмем средние значения функции $\widehat{\Delta}$, измеренной в моменты времени $j \geqslant\left(n^{0.01}+N\right) k+n^{0.01}$, соответствующие следующему большому блоку, относительно мер $\mathrm{P}_{\mathscr{G}}$ на собственном семействе в момент времени $m_{k}^{\prime \prime}$. Эти средние значения выражаются формулами, не зависящими от $\mathscr{G}$ (см. лемму 11.3), откуда следует, что динамики на различных больших блоках практически независимы.

ЛЕмма 11.3. Пусть $\mathbf{Z}$ обозначает сдвинутый большой блок:

$$
\mathbf{Z}=\sum_{j=\frac{1}{3} n^{0.01}}^{\frac{1}{3} n^{0.01}+N} \widehat{\Delta}_{j}
$$

Тогда для любого собственного стандартного семейства $\mathscr{G}$ в нулевой момент времени имеем

$$
\begin{aligned}
\mathrm{P}_{\mathscr{G}}(\mathbf{Z}) & =\mathscr{O}\left(\theta^{\frac{1}{4} n^{0.01}}\right), \\
\mathrm{P}_{\mathscr{G}}(\mathbf{Z} \otimes \mathbf{Z}) & =2 N \widehat{\mathbf{D}} \log R+\mathscr{O}(N), \\
\mathrm{P}_{\mathscr{G}}\left(\|\mathbf{Z}\|^{4}\right) & =\mathscr{O}\left(N R^{2} \log ^{3} n+N^{2} \log ^{2} n\right) .
\end{aligned}
$$

Здесъ $\widehat{\mathbf{D}}$ - матрица супердифбузии для дискретного времени, заданная в (2.1).

Теперь выведем теорему 8 (а) из этой леммы, используя характеристические функции. Пусть

$$
\phi_{m}(\xi)=\nu_{0}\left(\exp \left(\sum_{k=1}^{m} \frac{i\left\langle\xi, \mathbf{Z}_{k}\right\rangle}{\sqrt{n \log n}}\right)\right) .
$$

Согласно нашему обсуждению перед леммой 11.3, динамики внутри различных больших блоков почти независимы в том смысле, что

$$
\phi_{m}(\xi)=\prod_{k=1}^{m} \mathrm{P}_{\mathscr{G}_{k}}\left(\exp \left(\frac{i\left\langle\xi, \mathbf{Z}_{k}\right\rangle}{\sqrt{n \log n}}\right)\right)+\mathscr{O}\left(\theta_{1}^{n^{0.01}}\right)
$$

для некоторого $\theta_{1}<1$ и некоторых собственных стандартных семейств $\mathscr{G}_{k}$, каждое из которых взято в момент времени $m_{k-1}^{\prime \prime}$, т. е. за $\frac{1}{3} n^{0.01}$ столкновений до большого блока $\mathbf{Z}_{k}$. Теперь воспользуемся разложением Тейлора:

$$
\exp \left(\frac{i\left\langle\xi, \mathbf{Z}_{k}\right\rangle}{\sqrt{n \log n}}\right)=1+\frac{i\left\langle\xi, \mathbf{Z}_{k}\right\rangle}{\sqrt{n \log n}}-\frac{\left\langle\xi, \mathbf{Z}_{k}\right\rangle^{2}}{2 n \log n}+\mathscr{O}\left(\frac{\left\|\mathbf{Z}_{k}\right\|^{3}}{(n \log n)^{3 / 2}}\right) ;
$$

$\mathrm{P}_{\mathscr{G}_{k}}$-среднее первых трех членов по лемме 11.3 равно

$$
1-\frac{2\langle\widehat{\mathbf{D}} \xi, \xi\rangle N \log R}{2 n \log n}+o\left(\frac{N}{n}\right)=1-\frac{\langle\widehat{\mathbf{D}} \xi, \xi\rangle N}{2 n}+o\left(\frac{N}{n}\right) .
$$

Среднее последнего члена оценивается по неравенству Коши-Шварца:

$$
\begin{aligned}
\mathrm{P}_{\mathscr{G}_{k}}\left(\left\|\mathbf{Z}_{k}\right\|^{3}\right) & \leqslant\left[\mathrm{P}_{\mathscr{G}_{k}}\left(\left\|\mathbf{Z}_{k}\right\|^{2}\right) \mathrm{P}_{\mathscr{G}_{k}}\left(\left\|\mathbf{Z}_{k}\right\|^{4}\right)\right]^{1 / 2} \\
& =\mathscr{O}\left(N R \log ^{2} n+(N \log n)^{3 / 2}\right),
\end{aligned}
$$


так что его вкладом можно будет пренебречь. Заметим, что наши оценки не зависят от стандартного семейства $\mathrm{P}_{\mathscr{G}_{k}}$. Отсюда получаем

$$
\log \phi_{m}(\xi)=-\frac{m\langle\widehat{\mathbf{D}} \xi, \xi\rangle N}{2 n}+o\left(\frac{m N}{n}\right)
$$

и, полагая $m=n /\left(N+n^{0.01}\right)$, получаем

$$
\nu_{0}\left(\exp \left(\frac{i\left\langle\xi, \widetilde{\mathbf{q}}_{n}\right\rangle}{\sqrt{n \log n}}\right)\right) \rightarrow \exp \left(-\frac{\langle\widehat{\mathbf{D}} \xi, \xi\rangle}{2}\right)
$$

при $n \rightarrow \infty$. Отсюда следует слабая сходимость к нормальному распределению, которая утверждается в теореме 4. Наше доказательство отлично от приведенного в [2]; последнее использует спектральные свойства соответствующего трансфер-оператора.

Чтобы доказать слабую сходимость к броуновскому движению, которая утверждается в теореме 8 (a), нам нужны еще два свойства:

(i) слабая сходимость конечномерных распределений величины $\mathbf{q}_{s n} / \sqrt{n \log n}$ к конечномерным распределениям броуновского движения;

(ii) предкомпактность (см. ниже).

Чтобы вывести (i), предположим, что

$$
\frac{n_{1}}{n} \rightarrow s_{1}, \quad \frac{n_{2}}{n} \rightarrow s_{2}, \quad \ldots, \quad \frac{n_{k}}{n} \rightarrow s_{k} ;
$$

тогда такие же рассуждения, как и выше, показывают, что для любых $\xi_{1}$, $\xi_{2}, \ldots, \xi_{k}$

$$
\nu_{0}\left(\exp \left(\frac{i \sum_{j=1}^{k}\left\langle\xi_{j}, \widetilde{\mathbf{q}}_{n_{j}}\right\rangle}{\sqrt{n \log n}}\right)\right) \rightarrow \prod_{j=1}^{k} \exp \left(-\frac{\left\langle\widehat{\mathbf{D}} \eta_{j}, \eta_{j}\right\rangle\left(s_{j}-s_{j-1}\right)^{2}}{2}\right)
$$

где $s_{0}=0$ и $\eta_{j}=\sum_{p=1}^{j} \xi_{p}$. Из этой сходимости следует (i).

Остается показать, что семейство функций

$$
\left\{W_{n}(s)\right\}=\left\{S_{n s} / \sqrt{n \log n}\right\}, \quad 0<s<1,
$$

где $S_{n}=\sum_{j \leqslant n} \widehat{\Delta}_{j}$, является предкомпактным. В силу стандартных рассуждений (см., например, [30; гл. 2]) достаточно показать, что существует последовательность $\left\{\delta_{k}\right\}$, удовлетворяющая $\sum_{k} \delta_{k}<\infty$ и такая, что $\nu_{0}\left(\mathscr{M}_{K, n}\right) \rightarrow 0$ при $K \rightarrow \infty$ равномерно по $n$, где

$$
\mathscr{M}_{K, n}=\left\{\exists j, k: \frac{j}{2^{k}}<1 \text { и }\left|W_{n}\left(\frac{j+1}{2^{k}}\right)-W_{n}\left(\frac{j}{2^{k}}\right)\right|>K \delta_{k}\right\} .
$$

Пусть $\delta_{k}=1 / k^{2}$. Нужно оценить вероятность того, что

$$
\left|S_{n_{2}}-S_{n_{1}}\right| \geqslant \frac{K \sqrt{n \log n}}{k^{2}}
$$

где $n_{2}-n_{1} \sim n / 2^{k}$. Заметим, что $\left|S_{n_{2}}-S_{n_{1}}\right| \leqslant R n / 2^{k}$, откуда следует, что (11.7) невозможно, если $k^{2} / 2^{k}<1 / n$, в частности, если $k>C \log n$, где $C \gg 1-$ 
большая константа. При $k<C \log n$ воспользуемся леммой 11.3 и неравенством Маркова, чтобы оценить вероятность события (11.7) числом

$$
\begin{aligned}
\frac{k^{8} \nu_{0}\left(\left[S_{n_{2}}-S_{n_{1}}\right]^{4}\right)}{K^{4} n^{2} \log ^{2} n} & \leqslant C k^{8} \frac{\left(n_{2}-n_{1}\right) R^{2} \log ^{3} n+\left(n_{2}-n_{1}\right)^{2} \log ^{2} n}{K^{4} n^{2} \log ^{2} n} \\
& =\frac{C k^{8}}{K^{4}}\left(\frac{1}{2^{k} \log ^{99} n}+\frac{1}{4^{k}}\right)
\end{aligned}
$$

Поскольку (11.6) включает в себя $2^{k}$ интервалов размера $2^{-k}$, получаем

$$
\nu_{0}\left(\mathscr{M}_{K, n, k}\right) \leqslant \frac{C k^{8}}{K^{4}}\left(\frac{1}{\log ^{99} n}+\frac{1}{2^{k}}\right),
$$

где $\mathscr{M}_{(K, n, k)}$ обозначает часть множества (11.6), соответствующую данному $k$. Суммируя по $k \leqslant C \log n$, видим, что $\nu_{0}\left(\mathscr{M}_{K, n}\right) \leqslant C / K \rightarrow 0$ при $K \rightarrow \infty$ равномерно по $n$, откуда следует предкомпактность. Это завершает доказательство части (а) теоремы 8.

Доказательство части (б) проводится по той же схеме, что и доказательство части (а), с некоторыми изменениями, описываемыми далее. Во-первых, только $x$-компонента вектора смещения $\boldsymbol{\Delta}=\left(\Delta_{x}, \Delta_{y}\right)$ неограничена; его $y$-компонента ограничена и вполне регулярна (динамически гёльдеровски непрерывна). Поэтому переопределим $\widehat{\Delta}_{j}$ в $(11.1)$ следующим образом:

$$
\widehat{\Delta}_{j}=\left(\Delta_{j, x} \mathbf{1}_{\left\{\left|\Delta_{j, x}\right|<R\right\}}, \Delta_{j, y}\right),
$$

т. е. обрежем только $x$-компоненты. Теперь компоненты вектора $\mathbf{Z}=\left(\mathbf{Z}_{x}, \mathbf{Z}_{y}\right)$ в лемме 11.3 будут играть разные роли, и лемма изменяется следующим образом (см. доказательство в следующем разделе).

ЛЕмма 11.4. В условиях теоремы 8 (б) и в обозначениях леммы 11.3 имеем

$$
\begin{aligned}
\mathrm{P}_{\mathscr{G}}(\mathbf{Z}) & =\mathscr{O}\left(\theta^{\frac{1}{4} n^{0.01}}\right), \\
\mathrm{P}_{\mathscr{G}}\left(\mathbf{Z}_{x}^{2}\right) & =2 N \widehat{\mathbf{D}}_{x x} \log R+\mathscr{O}(N), \\
\mathrm{P}_{\mathscr{G}}\left(\mathbf{Z}_{y}^{2}\right) & =N \widehat{\mathbf{D}}_{y y}^{*}+\mathscr{O}(1), \\
\mathrm{P}_{\mathscr{G}}\left(\mathbf{Z}_{x} \mathbf{Z}_{y}\right) & =\mathscr{O}(N), \\
\mathrm{P}_{\mathscr{G}}\left(\mathbf{Z}_{x}^{4}\right) & =\mathscr{O}\left(N R^{2} \log ^{3} n+N^{2} \log ^{2} n\right), \\
\mathrm{P}_{\mathscr{G}}\left(\mathbf{Z}_{y}^{4}\right) & =\mathscr{O}\left(N^{2}\right) .
\end{aligned}
$$

Дальнейшее доказательство теоремы 8 (б) проводится, как доказательство части (а).

\section{2. Оценки моментов}

Здесь мы доказываем четыре леммы из предыдущего раздела, используя стандартные оценки моментов. Во-первых, в силу (9.11), (9.12) и следствия 9.2 имеем

$$
\nu_{0}\left(\widehat{\Delta}_{j}\right)=0, \quad \nu_{0}\left(\widehat{\Delta}_{j} \otimes \widehat{\Delta}_{j}\right)=\mathscr{O}(\log R)
$$


и для $i \neq j$

$$
\nu_{0}\left(\widehat{\Delta}_{i} \otimes \widehat{\Delta}_{j}\right)=\mathscr{O}\left(\theta^{|i-j|}\right) .
$$

Более того, вторую формулу в (12.1) можно уточнить следующим образом:

$$
\nu_{0}\left(\widehat{\Delta}_{j} \otimes \widehat{\Delta}_{j}\right)=2 \widehat{\mathbf{D}} \log R+\mathscr{O}(1)
$$

где $\widehat{\mathbf{D}}$ - матрица супердиффузии для дискретного времени, заданная в (2.1). Доказательство (12.3) проводится непосредственным интегрированием, подобным нашим вычислениям в разделе 10; на самом деле настоящий случай гораздо проще, так как строение рассеивателей периодическое; мы оставляем детали читателю (формула (12.3) по существу получена в [2], но без явного доказательства).

ДоКАЗАТЕЛЬСтво ЛЕммы 11.2. Оценим второй момент. Пусть $\mathbf{Q}=\sum_{k} \mathbf{Q}_{k}$. Заметим, что общее число членов в малых блоках равно $n^{1.01} / N$. В силу (12.2), (12.3) имеем

$$
\nu_{0}(\mathbf{Q} \otimes \mathbf{Q})=\mathscr{O}\left[(\log R) \frac{n^{1.01}}{N}+\frac{1}{R^{2}} \frac{n^{2.02}}{N^{2}}+\frac{n^{1.01}}{N}\right],
$$

где последний член получается из сходящегося ряда $\sum_{m} \theta^{m}$. Таким образом,

$$
\frac{\nu_{0}(\mathbf{Q} \otimes \mathbf{Q})}{n \log n} \rightarrow 0 \quad \text { при } n \rightarrow \infty,
$$

и лемма 11.2 следует из неравенства Чебышёва.

Далее, функция $\widehat{\Delta}$, определенная в $(11.1)$, динамически гёльдеровски непрерывна с параметром $\theta_{\widehat{\Delta}}<1$ (не зависящим от отсекающего значения $R$ ) и параметром $K_{\widehat{\Delta}}=\mathscr{O}(R)$; кроме того, $\|\widehat{\Delta}\|_{\infty}=\mathscr{O}(R)$. Таким образом, в силу теоремы 10 для любого собственного стандартного семейства $\mathscr{G}$ и любого $i \geqslant 0$

$$
\mathrm{P}_{\mathscr{G}}\left(\widehat{\Delta}_{i}\right)=\nu_{0}\left(\widehat{\Delta}_{i}\right)+\mathscr{O}\left(\theta^{i} R\right)=\mathscr{O}\left(\theta^{i} R\right)
$$

для некоторой константы $\theta<1$. Аналогично, из варианта теоремы 10 для многих времен наблюдения (см., например, [7; теорема 7.33]) следует, что для любых $p \geqslant 1$ и $i_{1} \leqslant \cdots \leqslant i_{p}$

$$
\mathrm{P} \mathscr{G}\left(\widehat{\Delta}_{i_{1}} \otimes \cdots \otimes \widehat{\Delta}_{i_{p}}\right)=\nu_{0}\left(\widehat{\Delta}_{i_{1}} \otimes \cdots \otimes \widehat{\Delta}_{i_{p}}\right)+\mathscr{O}\left(\theta^{i_{1}} R^{p}\right)
$$

Эта оценка нам потребуется только при $p=2$ и $p=4$.

ДокАЗАТЕЛЬСтво ЛЕммы 11.3. Оценка первого момента (11.3) следует из (12.4):

$$
\mathrm{P}_{\mathscr{G}}(\mathbf{Z})=\mathscr{O}\left(\theta^{\frac{1}{3} n^{0.01}} R\right)=\mathscr{O}\left(\theta^{\frac{1}{4} n^{0.01}}\right) .
$$

Оценка второго момента (11.4) следует из (12.2)-(12.5):

$$
\begin{aligned}
\mathrm{P}_{\mathscr{G}}(\mathbf{Z} \otimes \mathbf{Z})=\sum_{i, j} \mathrm{P}_{\mathscr{G}}\left(\widehat{\Delta}_{i} \otimes \widehat{\Delta}_{j}\right) & =\sum_{i, j} \nu_{0}\left(\widehat{\Delta}_{i} \otimes \widehat{\Delta}_{j}\right)+\mathscr{O}\left(N^{2} R^{2} \theta^{\frac{1}{3} n^{0.01}}\right) \\
& \sim 2 N \widehat{\mathbf{D}} \log R+\mathscr{O}(N),
\end{aligned}
$$

где суммирование идет по $i, j$, заданным в (11.2). 
Для доказательства оценки четвертого момента (11.5) положим

$$
\widehat{\Delta}_{j}=\left(\widehat{\Delta}_{j, x}, \widehat{\Delta}_{j, y}\right)
$$

по неравенству Коши-Шварца

$$
\|\mathbf{Z}\|^{4} \leqslant 2\left(\sum_{j} \widehat{\Delta}_{j, x}\right)^{4}+2\left(\sum_{j} \widehat{\Delta}_{j, y}\right)^{4},
$$

где суммирование идет по $j$, заданным в (11.2). Поскольку и $\widehat{\Delta}_{j, x}$, и $\widehat{\Delta}_{j, y}$ рассматриваются аналогично, мы опускаем индексы $x$ и $y$. Раскрывая четвертые степени, получаем суммы всевозможных произведений

$$
\widehat{\Delta}_{j_{1}} \widehat{\Delta}_{j_{2}} \widehat{\Delta}_{j_{3}} \widehat{\Delta}_{j_{4}}, \quad j_{1} \leqslant j_{2} \leqslant j_{3} \leqslant j_{4}
$$

В силу (12.5) имеем

$$
\mathrm{P}_{\mathscr{G}}\left(\widehat{\Delta}_{j_{1}} \widehat{\Delta}_{j_{2}} \widehat{\Delta}_{j_{3}} \widehat{\Delta}_{j_{4}}\right)=\nu_{0}\left(\widehat{\Delta}_{j_{1}} \widehat{\Delta}_{j_{2}} \widehat{\Delta}_{j_{3}} \widehat{\Delta}_{j_{4}}\right)+\mathscr{O}\left(\theta^{\frac{1}{3} n^{0.01}} R^{4}\right) .
$$

Зафиксируем большую константу $C_{1} \gg 1$ и разобьем произведения (12.6) на три категории в зависимости от промежутков между индексами

$$
D_{1}=j_{2}-j_{1}, \quad D_{2}=j_{3}-j_{2}, \quad D_{3}=j_{4}-j_{3} .
$$

Случай 1 (наиболее значимый): $\left|D_{i}\right| \leqslant C_{1} \log n$ для всех $i=1,2,3$. Тогда в силу неравенства Гёльдера имеем

$$
\left|\nu_{0}\left(\widehat{\Delta}_{j_{1}} \widehat{\Delta}_{j_{2}} \widehat{\Delta}_{j_{3}} \widehat{\Delta}_{j_{4}}\right)\right| \leqslant \nu_{0}\left(\widehat{\Delta}^{4}\right) \leqslant C \sum_{m=1}^{R} \frac{m^{4}}{m^{3}} \leqslant C R^{2},
$$

так что суммарный вклад таких членов является $\mathscr{O}\left(N R^{2} \log ^{3} n\right)$.

Случай 2 (умеренной значимости): $\left|D_{2}\right|>C_{1} \log n$ и $\left|D_{i}\right| \leqslant C_{1} \log n$ при $i=1,3$. Тогда в силу стандартных оценок корреляций [7; § 7.7], снова применяя неравенство Гёльдера, получаем

$$
\begin{aligned}
\nu_{0}\left(\widehat{\Delta}_{j_{1}} \widehat{\Delta}_{j_{2}} \widehat{\Delta}_{j_{3}} \widehat{\Delta}_{j_{4}}\right) & =\left[\nu_{0}\left(\widehat{\Delta}^{2}\right)\right]^{2}+\mathscr{O}\left(R^{4} \theta^{C_{1} \log n}\right) \\
& =\mathscr{O}\left(\log ^{2} R\right)+\mathscr{O}\left(n^{-100}\right) .
\end{aligned}
$$

Тем самым суммарный вклад таких членов является $\mathscr{O}\left(N^{2} \log ^{2} n\right)$.

Случай 3 (наименее значимый): $\left|D_{1}\right|>C_{1} \log n$ или $\left|D_{3}\right|>C_{1} \log n$. В этом случае, поскольку $\nu_{0}(\widehat{\Delta})=0$, оценки корреляций легко дают верхнюю оценку порядка $\mathscr{O}\left(n^{-100}\right)$.

Лемма 11.3 доказана.

ДоКАЗАТЕЛЬСТВо ЛЕммы 11.1. Причиной того, что $\widetilde{\mathbf{q}}_{n} \neq \sum_{j=0}^{n-1} \widehat{\Delta}_{j}$, является то, что некоторые $\boldsymbol{\Delta}_{j}$ принимают большие значения, превосходящие $R$. Разобьем наше рассуждение на две части. Сначала положим

$$
R^{\prime}=\sqrt{n} \log \log n
$$


и покажем, что значения $\boldsymbol{\Delta}_{j}$, превосходящие $R^{\prime}$, можно не рассматривать, так как их вероятности пренебрежимо малы. Действительно, в силу (3.1) имеем

$$
\nu_{0}\left(\exists j \leqslant n:\left\|\boldsymbol{\Delta}_{j}\right\|>R^{\prime}\right)=\mathscr{O}\left((\log \log n)^{-2}\right) \rightarrow 0 .
$$

Далее покажем, что умеренно большие значения $\boldsymbol{\Delta}_{j}$ (между $R$ и $R^{\prime}$ ), хотя и встречаются довольно часто, стремятся сократиться друг с другом. Пусть

$$
\widetilde{\Delta}_{j}=\boldsymbol{\Delta}_{j} \mathbf{1}_{\left\{R \leqslant\left\|\Delta_{j}\right\| \leqslant R^{\prime}\right\}}, \quad \widetilde{S}_{m}=\sum_{j=0}^{m-1} \widetilde{\Delta}_{j}
$$

Заметим, что

$$
\nu_{0}\left(\widetilde{\Delta}_{j}\right)=0, \quad \nu_{0}\left(\widetilde{\Delta}_{j} \otimes \widetilde{\Delta}_{j}\right) \leqslant C \sum_{m=R}^{R^{\prime}} \frac{m^{2}}{m^{3}} \leqslant C \log \log n .
$$

Рассуждая как в доказательстве оценки второго момента в лемме 11.3, получаем для любого $m \leqslant n$ неравенство

$$
\nu_{0}\left(\widetilde{S}_{m} \otimes \widetilde{S}_{m}\right) \leqslant C m \log \log n,
$$

и, значит, в силу неравенства Чебышёва для любого $\epsilon>0$

$$
\nu_{0}\left(\left\|\widetilde{S}_{m}\right\| \geqslant \epsilon \sqrt{n \log n}\right) \rightarrow 0
$$

равномерно по $m$. Но нужно получить более сильную оценку:

$$
\nu_{0}\left(\max _{1 \leqslant m \leqslant n}\left\|\widetilde{S}_{m}\right\| \geqslant \epsilon \sqrt{n \log n}\right) \rightarrow 0
$$

С этой целью покажем, что множества

$$
\mathscr{M}_{m, \epsilon}=\left\{X \in \mathscr{M}:\left\|\widetilde{S}_{m}\right\| \geqslant \epsilon \sqrt{n \log n}\right\}
$$

стремятся сильно перекрывать друг друга. Сначала заметим, что

$$
\nu_{0}\left(\mathscr{M}_{m, \epsilon} \backslash \mathscr{M}_{m-1, \epsilon}\right) \leqslant \nu_{0}\left(\widetilde{\Delta}_{m-1} \neq 0\right) \leqslant \frac{C \log ^{200} n}{n},
$$

где последняя оценка следует из (3.1). Далее, покажем, что

$$
\nu_{0}\left(\left(\mathscr{M}_{m, \epsilon} \backslash \mathscr{M}_{m-1, \epsilon}\right) \backslash \mathscr{M}_{n, \epsilon / 2}\right) \leqslant \delta_{n} \nu_{0}\left(\mathscr{M}_{m, \epsilon} \backslash \mathscr{M}_{m-1, \epsilon}\right),
$$

где $\delta_{n} \rightarrow 0$ при $n \rightarrow \infty$. Тогда из простого суммирования по $m=1, \ldots, n$ будет следовать, что

$$
\nu_{0}\left(\bigcup_{m=1}^{n} \mathscr{M}_{m, \epsilon}\right) \leqslant \delta_{n}+\nu_{0}\left(\mathscr{M}_{n, \epsilon / 2}\right)
$$

Сочетая это с (12.7), получим (12.8).

Наше доказательство неравенства (12.9) напоминает принцип отражения в теории случайных блужданий. Заметим, что каждая точка множества в левой части (12.9) удовлетворяет (одновременно) трем условиям:

$$
\widetilde{\Delta}_{m-1} \neq 0, \quad\left\|\sum_{j=0}^{m-1} \widetilde{\Delta}_{j}\right\| \geqslant \epsilon \sqrt{n \log n}, \quad\left\|\sum_{j=m}^{n-1} \widetilde{\Delta}_{j}\right\| \geqslant \frac{1}{2} \epsilon \sqrt{n \log n} .
$$


Поскольку $\widetilde{\Delta}_{m-1} \neq 0$, имеем $\left\|\boldsymbol{\Delta}_{m-1}\right\| \geqslant R=\sqrt{n} / \log ^{100} n$. Стандартным свойством газа Лоренца является то, что как только $\boldsymbol{\Delta}_{m-1}$ велико, то, как правило, $\left\|\boldsymbol{\Delta}_{m}\right\| \sim\left\|\boldsymbol{\Delta}_{m-1}\right\|^{1 / 2}$, тогда $\left\|\boldsymbol{\Delta}_{m+1}\right\| \sim\left\|\boldsymbol{\Delta}_{m-1}\right\|^{1 / 4}$, и т. д. Точнее, существуют такие $p, q>0$, что для любого большого $C_{2}>0$ существует такое $C_{3}>0$, что

$$
\begin{gathered}
\nu_{0}\left(\left\|\boldsymbol{\Delta}_{m-1}\right\| \geqslant R \text { и } \max _{0 \leqslant k \leqslant C_{2} \log n}\left\|\boldsymbol{\Delta}_{m+k}\right\|>\left\|\boldsymbol{\Delta}_{m-1}\right\|^{1-q}\right) \\
\leqslant C_{3} R^{-p} \nu_{0}\left(\left\|\boldsymbol{\Delta}_{m-1}\right\| \geqslant R\right) \leqslant C_{3} R^{-(2+p)}
\end{gathered}
$$

(см. [29; лемма 5.1]), и эта мера, очевидно, пренебрежимо мала. Таким образом, можно предполагать, что как только $\widetilde{\Delta}_{m-1} \neq 0$, то $\widetilde{\Delta}_{m+k}=0$ для всех $k=$ $0,1, \ldots, C_{2} \log n$.

При $k>C_{2} \log n$ корреляции между $\widetilde{\Delta}_{j}, j \leqslant m$, и $\widetilde{\Delta}_{m+k}$ малы, скажем, они $<n^{-200}$, если $C_{2}$ достаточно велико. Таким образом, имеем

$$
\begin{aligned}
\nu_{0}\left(\left(\mathscr{M}_{m, \epsilon} \backslash \mathscr{M}_{m-1, \epsilon}\right) \backslash \mathscr{M}_{n, \varepsilon / 2}\right) & =\nu_{0}\left(\mathscr{M}_{m, \epsilon} \backslash \mathscr{M}_{m-1, \epsilon}\right) \\
\times \nu_{0}\left(\left\|\sum_{j=m+C_{2} \log n}^{n-1} \widetilde{\Delta}_{j}\right\|\right. & \left.\geqslant \frac{1}{2} \epsilon \sqrt{n \log n}\right)+\mathscr{O}\left(n^{-100}\right),
\end{aligned}
$$

причем второй множитель в правой части сходится к нулю равномерно по $m$ в силу (12.7). Это завершает доказательство неравенства (12.9). Тем самым доказана оценка (12.8), откуда вытекает утверждение леммы 11.1.

ДокАЗАТЕЛЬСТво ЛЕмМы 11.4. Оценки, имеющие дело только с $\mathbf{Z}_{x}$, выводятся таким же образом, как лемма 11.3. Оценки, имеющие дело только с $\mathbf{Z}_{y}$, вполне стандартны (см., например, [6] или [31; §9], или [7; гл. 7]), поскольку $\Delta_{y}$ ограничена и динамически гёльдеровски непрерывна. Остается оценить член с перекрестным произведением

$$
\mathrm{P}_{\mathscr{G}}\left(\mathbf{Z}_{x} \mathbf{Z}_{y}\right)=\sum_{i, j} \mathrm{P}_{\mathscr{G}}\left(\widehat{\Delta}_{i, x} \Delta_{j, y}\right) .
$$

Как и ранее, обрежем большую компоненту $\widehat{\Delta}_{i, x}$ следующим образом:

$$
\widehat{\Delta}_{i, x}=\widehat{\Delta}_{i, x}^{\prime}+\widehat{\Delta}_{i, x}^{\prime \prime}, \quad \widehat{\Delta}_{i, x}^{\prime}=\widehat{\Delta}_{i, x} \mathbf{1}_{\left\{\left|\widehat{\Delta}_{i, x}\right|<H\right\}}
$$

(отсекающее значение $H=H_{i j}$ будет выбрано ниже). Тогда снова

$$
\mathrm{P}_{\mathscr{G}}\left(\widehat{\Delta}_{i, x}^{\prime} \Delta_{j, y}\right)=\mathscr{O}\left(H \theta_{1}^{|i-j|}\right)
$$

для некоторого $\theta_{1}<1$, тогда как в силу $(12.4)$ и ограниченности $\Delta_{j, y}$ имеем

$$
\begin{aligned}
\left|\mathrm{P}_{\mathscr{G}}\left(\widehat{\Delta}_{i, x}^{\prime \prime} \Delta_{j, y}\right)\right| & \leqslant C \mathrm{P}_{\mathscr{G}}\left(\left|\widehat{\Delta}_{i, x}^{\prime \prime}\right|\right) \leqslant C \nu_{0}\left(\left|\widehat{\Delta}_{i, x}^{\prime \prime}\right|\right)+C \theta^{\frac{1}{3} n^{0.01}} R \\
& \leqslant C H^{-1}+C \theta^{\frac{1}{4} n^{0.01}} .
\end{aligned}
$$

Выбирая $H=\theta_{1}^{-\frac{1}{2}|i-j|}$, получаем

$$
\mathrm{P} \mathscr{G}\left(\widehat{\Delta}_{i, x} \Delta_{j, y}\right)=\mathscr{O}\left(\theta^{\frac{1}{2}|i-j|}\right)+C \theta^{\frac{1}{4} n^{0.01}},
$$

откуда следует $\mathrm{P}_{\mathscr{G}}\left(\mathbf{Z}_{x} \mathbf{Z}_{y}\right)=\mathscr{O}(N)$, как утверждалось. 
Наконец, очертим схему доказательства предложений 2.1-2.3. Доказательства предложений 2.1 и 2.3 проводятся по схеме доказательства теоремы 8. А именно, сначала отсечем аномально большие значения функции $\boldsymbol{\Delta}_{\varepsilon}$, если это необходимо (т. е. если $t<\varepsilon \log ^{100} \varepsilon$ ), и применим технику больших и малых блоков. Заметим, что стандартное доказательство центральной предельной теоремы для случая конечного горизонта здесь не работает по следующей причине. Это доказательство опирается на то, что $\nu_{0}\left(\widetilde{\mathbf{q}}_{n}^{4}\right)$ имеет тот же порядок, что и $\left[\nu_{0}\left(\widetilde{\mathbf{q}}_{n}^{2}\right)\right]^{2}$. В данном же случае $\nu_{0}\left(\widetilde{\mathbf{q}}_{n}^{2}\right)=\mathscr{O}(n|\log \varepsilon|)$, но оценка четвертого момента более сложная. Как мы знаем (см. доказательство леммы 11.3), эта оценка основана на рассмотрении трех случаев: случай 1 дает вклад $\sim n \varepsilon^{-1}$, случай 2 дает вклад $\sim n^{2} \log ^{2} \varepsilon$, а случай 3 незначимый. Видим, что для больших $n$ случай 2 доминирует и приводит к желаемому соотношению $\nu_{0}\left(\widetilde{\mathbf{q}}_{n}^{4}\right) \sim\left[\nu_{0}\left(\widetilde{\mathbf{q}}_{n}^{2}\right)\right]^{2}$. Но для малых $n$ доминирует случай 1 (в точности как в доказательстве леммы 11.3), и это вызывает аномальную диффузию.

Доказательство предложения 2.2 проводится следующим образом. Первая формула выводится аналогично теореме $6($ г), вторая формула - аналогично предложению 3.1, а третья формула - аналогично теореме 2 (г).

\section{Список литературы}

[1] N. I. Chernov, G. L. Eyink, J. L. Lebowitz, Ya. G. Sinai, "Steady-state electrical conduction in the periodic Lorentz gas", Comm. Math. Phys., 154:3 (1993), 569-601.

[2] D. Szász, T. Varjú, "Limit laws and recurrence for the planar Lorentz process with infinite horizon", J. Statist. Phys., 129:1 (2007), 59-80.

[3] H. A. Lorentz, "The motion of electrons in metallic bodies", Proc. Amst. Acad., 7 (1904), 438-453.

[4] Я.Г. Синай, "Динамические системы с упругими отражениями. Эргодические свойства рассеивающих бильярдов", УМН, 25:2 (1970), 141-192; англ. пер.: Ya. G. Sinai, "Dynamical systems with elastic reflections", Russian Math. Surveys, 25:2 (1970), 137-189.

[5] G. Gallavotti, D. S. Ornstein, "Billiards and Bernoulli schemes", Comm. Math. Phys., 38:2 (1974), 83-101.

[6] Л.А. Бунимович, Я.Г. Синай, Н.И. Чернов, "Статистические свойства двумерных гиперболических биллиардов", УМН, 46:4 (1991), 43-92; англ. пер.: L. A. Bunimovich, Ya. G. Sinai, N. I. Chernov, "Statistical properties of two-dimensional hyperbolic billiards", Russian Math. Surveys, 46:4 (1991), 47-106.

[7] N. Chernov, R. Markarian, "Chaotic billiards", Math. Surveys Monogr., 127, Amer. Math. Soc., Providence, RI, 2006.

[8] L. A. Bunimovich, Ya. G. Sinai, "Statistical properties of Lorentz gas with periodic configuration of scatterers", Comm. Math. Phys., 78:4 (1981), 479-497.

[9] Lai-Sang Young, "Statistical properties of dynamical systems with some hyperbolicity", Ann. of Math. (2), 147:3 (1998), 585-650.

[10] N. I. Chernov, G. L. Eyink, J. L. Lebowitz, Ya. G. Sinai, "Derivation of Ohm's law in a deterministic mechanical model", Phys. Rev. Lett., 70:15 (1993), 2209-2212.

[11] B. Moran, W. G. Hoover, S. Bestiale, "Diffusion in a periodic Lorentz gas", J. Statist. Phys., 48:3-4 (1987), 709-726.

[12] N. Chernov, "Sinai billiards under small external forces", Ann. Henri Poincaré, 2:2 (2001), 197-236.

[13] N. Chernov, "Sinai billiards under small external forces. II", Ann. Henri Poincaré, 9:1 (2008), 91-107. 
[14] N. Chernov, D. Dolgopyat, "Diffusive motion and recurrence on an idealized Galton board", Phys. Rev. Lett., 99:3 (2007), paper 030601 (4 pages).

[15] N. Chernov, D. Dolgopyat, "The Galton board: limit theorems and recurrence", $J$. Amer. Math. Soc., 22 (2009), 821-858.

[16] F. Galton, Natural Inheritance, Macmillan, 1889.

[17] N. Chernov, D. Dolgopyat, "Brownian Brownian Motion. I", Mem. Amer. Math. Soc., 198, № 927, 2009.

[18] P. M. Bleher, "Statistical properties of two-dimensional periodic Lorentz gas with infinite horizon", J. Statist. Phys., 66:1-2 (1992), 315-373.

[19] J. Marklof, A. Strömbergsson, "Kinetic transport in the two-dimensional periodic Lorentz gas", Nonlinearity, 21:7 (2008), 1413-1422.

[20] J. Marklof, A. Strömbergsson, "The distribution of free path lengths in the periodic Lorentz gas and related lattice point problems", Ann. of Math. (to appear); arxiv.org: 0706.4395, 2007.

[21] N. Chernov, "Decay of correlations and dispersing billiards", J. Statist. Phys., 94:3-4 (1999), 513-556.

[22] Lai-Sang Young, "Dimension, entropy and Lyapunov exponents", Ergodic Theory Dynam. Systems, 2:1 (1982), 109-124.

[23] I. Melbourne, A. Török, "Statistical limit theorems for suspension flows", Israel J. Math., 144:2 (2004), 191-209.

[24] M. P. Wojtkowski, "W-flows on Weyl manifolds and Gaussian thermostats", J. Math. Pures Appl. (9), 79:10 (2000), 953-974.

[25] N. Chernov, H.-K. Zhang, "Billiards with polynomial mixing rates", Nonlinearity, 18:4 (2005), 1527-1553.

[26] Ya. B. Pesin, "Dynamical systems with generalized hyperbolic attractors: hyperbolic, ergodic and topological properties", Ergodic Theory Dynam. Systems, 12:1 (1992), 123-151.

[27] Е.А. Сатаев, "Инвариантные меры для гиперболических отображений с особенностями", УМН, 47:1 (1992), 147-202; англ. пер.: E. A. Sataev, "Invariant measures for hyperbolic maps with singularities", Russian Math. Surveys, 47:1 (1992), 191-251.

[28] N. Chernov, H.-K. Zhang, "On statistical properties of hyperbolic systems with singularities" (to appear); http://www. math.uab.edu/ chernov/papers/Chernovzhang1.pdf.

[29] N. Chernov, H.-K. Zhang, "Improved estimates for correlations in billiards", Comm. Math. Phys., 277:2 (2008), 305-321.

[30] P. Billingsley, Convergence of probability measures, Wiley, New York-London-Sydney, 1968; рус. пер.: П. Биллингсли, Сходимость вероятностных мер, Наука, Физматлит, М., 1977.

[31] D. Dolgopyat, "Limit theorems for partially hyperbolic systems", Trans. Amer. Math. Soc., 356:4 (2004), 1637-1689.

Д. И. Долгопят (D. I. Dolgopyat)

University of Maryland,

Поступила в редакцию

College Park

E-mail: dolgop@math.psu.edu

\section{Н. И. Чернов (N. I. Chernov)}

University of Alabama

at Birmingham

E-mail: chernov@math.uab.edu 